\title{
The Effect of Orthographic Systems on the Developing Reading System: Typological and Computational Analyses
}

\author{
Alastair C. Smith \\ Max Planck Institute for Psycholinguistics
}

\author{
Padraic Monaghan \\ Lancaster University
}

\author{
Falk Huettig \\ Max Planck Institute for Psycholinguistics and Radboud University
}

\begin{abstract}
Orthographic systems vary dramatically in the extent to which they encode a language's phonological and lexico-semantic structure. Studies of the effects of orthographic transparency suggest that such variation is likely to have major implications for how the reading system operates. However, such studies have been unable to examine in isolation the contributory effect of transparency on reading because of covarying linguistic or sociocultural factors. We first investigated the phonological properties of languages using the range of the world's orthographic systems (alphabetic, alphasyllabic, consonantal, syllabic, and logographic), and found that, once geographical proximity is taken into account, phonological properties do not relate to orthographic system. We then explored the processing implications of orthographic variation by training a connectionist implementation of the triangle model of reading on the range of orthographic systems while controlling for phonological and semantic structure. We show that the triangle model is effective as a universal model of reading, able to replicate key behavioral and neuroscientific results. The model also generates new predictions deriving from an explicit description of the effects of orthographic transparency on how reading is realized and defines the consequences of orthographic systems on reading processes.
\end{abstract}

Keywords: orthographic transparency, phonological processing, orthographic systems, literacy, neural network modeling

Supplemental materials: http://dx.doi.org/10.1037/rev0000257.supp

Current dominant psychological and cognitive neuroscientific descriptions of how we read, how we acquire this ability, and the broader cognitive consequences of its acquisition are almost entirely built upon the study of alphabetic literates, where readers transform series of letters (with a componential correspondence) to speech sound-based segment-sized units (henceforth phonology, or phonological form or structure) of words. The world's major orthographic systems (alphabetic, consonantal, syllabic, alphasyllabic, and logographic) vary dramatically in the manner in which they encode a language's phonological and semantic structure (i.e., their semantic or phonological transparency; Comrie, 2013). Recent years have seen an increasing broadening of attention within

This article was published Online First August 10, 2020.

Alastair C. Smith, Psychology of Language, Max Planck Institute for Psycholinguistics; Padraic Monaghan, Department of Psychology, Lancaster University; Falk Huettig, Psychology of Language, Max Planck Institute for Psycholinguistics, and Donders Institute for Brain, Cognition, and Behaviour, Radboud University.

Falk Huettig is now at Centre for Language Studies, Radboud University.

Correspondence concerning this article should be addressed to Padraic Monaghan, Department of Psychology, Lancaster University, Lancaster LA1 4YF, United Kingdom. E-mail: p.monaghan@lancaster.ac.uk experimental reading research to alternate orthographic systems. Across the world's literate population, there are over 1 billion logographic literates, over 500 million alphasyllabic literates, in addition to many hundreds of million consonantal and syllabic literates (see online supplemental materials for details of how population size estimates were calculated). However, given this variety of orthographic systems, existing reading models are largely based on reading in English or other alphabetical orthographies (Chang, Monaghan, \& Welbourne, 2019; Coltheart, Rastle, Perry, Langdon, \& Ziegler, 2001; Harm \& Seidenberg, 2004; Perry, Ziegler, \& Zorzi, 2010; see Share, 2008 for discussion); yet, the variety of ways in which orthographic systems reflect representations of words are likely to have profound consequences for how reading is acquired and the effect that orthography has on the cognitive mechanisms recruited for reading.

In this article we present a series of models of reading that implement the inherent differences between orthographic systems across the world's major orthographic systems. We first provide a review of the literature that describes the effect that orthographic variation has on the trajectory of reading acquisition, the impact of orthographic systems on the processes involved in the mature reading system, and the differential effects of literacy on cognitive processing more broadly. We then examine potential systematic variation across languages in relationships between orthographic system and the phonological and semantic structure of languages 
(Frost, 2012; Taylor \& Olson, 1995), providing a typological analysis of orthographic and phonological structure of languages. Finally, we present our implemented model of reading, based on the triangle modeling tradition in simulating the reading process (e.g., Harm \& Seidenberg, 2004), and demonstrate how the variation found in the world's orthographic systems affects computations in both the manner in which reading is acquired and how the reading system operates after extended experience of reading. The modeling demonstrates that a comprehensive understanding of the acquisition and operation of reading requires a full consideration of variation in orthographies. Our modeling enables an explicit test of theoretical views on how orthographic variation affects the reading system, and provides an explanation for how behavioral distinctions in reading development emerge as a consequence of these variations in the way in which sound and meaning distinctions are conveyed in the orthographic system.

\section{Orthographic Diversity}

Five categories of orthographic system are typically defined to describe the range of extant orthographic systems found globally: alphabetic, consonantal, syllabic, alphasyllabic, and logographic (Comrie, 2013). The extent to which the written form reflects the phonology of the word-the transparency of the orthographyvaries greatly over these systems, and we describe them in order of transparency, from greatest to least.

Alphabetic systems are the most frequent orthographic system in use in the 21st Century world, existing throughout Europe, the Americas, Australasia and many portions of Asia and sub-Saharan Africa. Within such systems the basic unit of representation are graphemes (grapheme: a letter or set of letters) that correspond (more or less) closely to individual segment-sized, speech soundbased units (phonemes), that is, orthography contains detailed information regarding the fine grained phonological structure of the language. Alphabetic systems vary in the granularity and regularity of mappings between orthography and phonology. Within shallow alphabetic systems, such as Finnish or SerboCroatian, there is close to perfect one to one correspondence between individual phonemes and graphemes, whereas in deep alphabetic systems such as English there are deviations in regularity (i.e., the extent to which a letter or set of letters maps onto the same phoneme or set of phonemes, e.g., the "I" in flint or pint) and granularity (that is, the number of letters that combine to relate to sounds in the word, e.g., cot, yacht) of mappings.

Consonantal systems possess very similar structural properties to alphabetic systems yet with the defining feature that they represent only consonants, not vowels. Such systems (e.g., Arabic, Hebrew) are prevalent in the Middle East and northern Africa. It should be noted, however, that many of today's consonantal systems also exist in alphabetic or alphasyllabic form because of the addition of diacritics that are used to indicate the presence of a particular vowel, inclusion of diacritics is particularly common during early stages of literacy training in such orthographic systems (Abu-Rabia \& Taha, 2006; Ravid, 2006; Share, 2008). Nevertheless, languages such as Arabic and Hebrew are typically written without diacritics.

The basic grapheme within alphasyllabic systems indicates a consonant; however, information regarding the vowel is also encoded in such systems either in the form of diacritics added to the consonant preceding or after the vowel or through a predefined transformation of the preceding or subsequent consonant's representation as a grapheme. Such systems are widespread (Comrie, 2013) but concentrated most intensely in India (e.g., Devanagari used for languages such as Hindi and Bengali) and South East Asia (e.g., Thai).

Syllabic systems provide a fourth category, within which the functional unit is the syllable. In its idealized form a single grapheme corresponds to each syllable within the phonology. Japanese hiragana and katakana are examples of such systems, however, pure syllabic systems are rare, with contemporary Japanese largely communicated in a mixed logographic-syllabic form (the syllabic hiragana and katakana, and the logographic kanji of Chinese origin; Smith, 1996).

The basic representational unit in logographic systems is the morpheme and, therefore, contrasts with other orthographic systems in which representational units are related to phonological properties. Chinese is the only logographic script in widespread use today (Treiman \& Kessler, 2005). Though some Chinese characters are pictograms or ideograms, most (82\%; Zhou, 1978) Chinese words are compounds of two (or more) characters that contain phonetic radicals and semantic radicals that, respectively, provide some (but rarely fully reliable) information regarding the word's phonetic or semantic properties.

\section{Effects of Orthographic Transparency}

In all these orthographic types, readers can evidently learn to map from written to spoken and meaning representations for words. However, this diversity in orthographic structure can have quantitative and qualitative effects on acquisition and processing of the reading system and may also have wider cognitive implications for the way in which the reading system integrates with preexisting language processing networks.

\section{Effects on Acquisition}

Probably the domain in which there is greatest understanding and consensus regarding the impact of orthographic transparency on reading is in the rate of acquisition of phonological decoding abilities (Snowling \& Hulme, 2005). A consistent problem faced by researchers who aim to compare the effects of orthographic structure across contrasting systems, is that systems and populations also vary across many other dimensions, such as language factors, for example, phonological complexity of the language, visual complexity of the orthography, word order, morphological complexity, and syntactic structure; or sociocultural factors, for example, teaching methods, educational background and student motivation (see Ziegler \& Goswami, 2005, for discussion). Nevertheless, there have been attempts to characterize differences that are a consequence only of the orthography (see Seidenberg, 2011, 2013, 2017, for reviews). It has been found that children learning a deep alphabetic system such as English require 4-5 years of literacy training to reach $90 \%$ accuracy on nonword reading tasks (Goswami, Gombert, \& De Barrera, 1998) whereas children learning to read a shallow system such as Finnish reach this level of attainment after their first year of tuition (Seymour, Aro, \& Erskine, 2003).

Although it is very difficult to control for factors beyond the orthographic system variation, studies that aim to minimize the 
impact of such factors have consistently shown that increased phonological transparency coincides with increased phonological decoding acquisition rates. For example, Bruck, Genesee, and Caravolas (1997) compared groups of English speaking and French speaking children from the same region of Canada on monosyllabic nonword and word reading performance following a year of literacy tuition. They found that the English speaking population displayed $24 \%$ lower performance on word reading tasks and $27 \%$ lower performance on nonword reading tasks compared with their French speaking counterparts, which was suggested to be because of the greater orthographic transparency of French than English. A similar study conducted between children from the same region of the United Kingdom learning to read either Welsh or English found that children learning Welsh, a shallower orthographic system than English, similarly displayed increased performance on nonword and word reading when controlling for training exposure (Hanley, Masterson, Spencer, \& Evans, 2004).

Direct comparisons across orthographic types are less common still, and prone to increased confounds of linguistic and sociocultural factors; however, the time required to reach similar levels of reading proficiency across populations is highly suggestive of transparency leading to faster rates of decoding acquisition. Asfaha, Kurvers, and Kroon (2009) compared reading acquisition rates over the first year of literacy acquisition across four populations learning to read one of four African languages in either a syllabic (Ge'ez) or alphabetic (Latin) script, and observed an increased rate of acquisition of the syllabic orthographies, which contrasts somewhat with other studies on effects of orthographic transparency. However, for acquisition of a logographic system, the results are again consistent with transparency affecting learning to read. Chinese was found to result in a slow acquisition rate as it requires intensive training over the first 6 years of schooling for children to learn the 2,500 foundational characters required to support proficient Chinese reading (Cheung \& Ng, 2003). This point is further supported by the fact that Chinese children on mainland China nowadays rapidly (within a few months) acquire the alphabetic Hanyu Pinyin system that is taught at the same time as the traditional logographic system in PR China. In other words, children in mainland China become fully alphabetic literate a long time before they become literate logographic readers.

Studies of reading acquisition that have examined predictors of reading proficiency that extend beyond phonological decoding (e.g., reading fluency) demonstrate variation in the influence of phonological, semantic, and morphological factors across orthographies. Ziegler et al. (2010) showed that across alphabetic systems of varying orthographic depth, a set of predictors of reading performance were universally informative; yet, varied systematically in their relative influence as a function of orthographic transparency. Specifically, phonological awareness was a stronger predictor in less transparent alphabetic scripts. A similar study (Vaessen et al., 2010) also examined predictors of proficiency, in this case of reading fluency, over the course of literacy training across three orthographies that differed in their orthographic depth (Hungarian, Dutch, and Portuguese). Vaessen et al. also found a consistency across alphabetic systems with phonological awareness and rapid naming contributing across all orthographies at similar stages of development yet with orthographic depth affecting the rate of development. Together these studies suggest a quantitative rather than qualitative difference in the cognitive processes engaged in reading acquisition across alphabetic systems that differ in orthographic depth. Whether such a statement extends to differences that may exist across orthographic systems of differing categories appears less clear. Results presented in CohenMimran (2009) show that phonological awareness did not predict reading fluency in less transparent orthographies such as pointed (alphasyllabic) or unpointed (consonantal) Hebrew scripts, whereas morphological measures were good predictors for both, and semantic measures for unpointed performance. ${ }^{1}$ However, Bar-Kochva and Breznitz (2014) showed that phonological awareness can predict reading fluency in alphasyllabic and consonantal readers. Furthermore, Tong and McBride-Chang (2010) tested reading performance for children learning to read in Chinese and English concurrently. Their results show that predictors of variation in reading performance in Chinese and English were stable across age groups yet differed across scripts, with morphological measures predicting variation in Chinese but not English reading, while phonological awareness predicted reading in English but not Chinese (see also Tong, McBride-Chang, Shu, \& Wong, 2009). Zhou et al. (2018) observed in Chinese-speaking children in Hong Kong that phonological awareness was an important predictor for both learning to read English and Chinese but that a wider range of skills such as morphological awareness and visual skills were important for Chinese reading. It is possible, therefore, that qualitative in addition to quantitative differences in the cognitive processes recruited when learning to read may emerge from differences in the constraints placed on the cognitive system by different orthographic structures.

Although, many studies that examine the effects of orthographic transparency on reading acquisition report a delay in decoding ability in less transparent systems, the extent to which transparency impacts on comprehension skills is less clear as comprehension measures are often not included in such studies (Seidenberg, 2011, 2013). For example, in Turkish, a shallow orthographic system, a high proficiency in decoding is achieved very early in reading development; however, comprehension ability is delayed (Durgunoglu, 2006). Similarly, English speaking children have been shown to regularly understand the meaning of written words they are unable to decode accurately (Nation, 2009; Nation \& Cocksey, 2009). This is potentially reflected in the Welsh-English study previously described which also found that English readers outperformed Welsh readers in their comprehension abilities (Hanley et al., 2004).

In summary, the data regarding effects of orthographic transparency on the ability to learn orthographic to phonological mappings generally demonstrates that transparency aids acquisition. However, the effect of transparency on comprehension remains an underexplored issue.

\section{Effects on Processing}

There are two theoretically motivated paths via which orthographic information could activate semantic and phonological representations, which are consistent with most implemented models

\footnotetext{
${ }^{1}$ It should be noted that pointed Hebrew script is used for the first year of formal literacy training in Israeli schools (Frost, 2012; Shany, Bar-On, \& Katzir, 2012; Weiss, Katzir, \& Bitan, 2015).
} 
of word reading (Chang \& Monaghan, 2019; Taylor, Duff, Woollams, Monaghan, \& Ricketts, 2015). Activations can be either direct, where correspondences between orthographic and phonological forms and between orthographic and semantic forms are acquired with resources dedicated to forming these mappings. Alternatively, activations could be indirect, where correspondences between orthography and phonology are mediated by the words' semantics, where phonology to semantic representations are acquired before literacy, or from orthography to semantics, via phonological representations. The orthographic depth hypothesis (ODH; Frost, Katz, \& Bentin, 1987; Katz \& Feldman, 1981) states that the transparency of the orthography will dictate the extent to which direct and indirect paths are engaged for reading, and that this will be determined by the degree of systematicity between orthographic and phonological or semantic representations.

The strongest interpretation of the ODH contends that readers of shallow alphabetic systems will rapidly acquire word naming fluency - the orthographic to phonological mappings-along a direct route because of the regularity of the grapheme to phoneme correspondences. However, reading comprehension-so mapping from orthography to semantics - will largely depend on the indirect route via phonology, because orthography to semantics is a largely arbitrary mapping, which is hard to learn, and so activation of meaning will derive from the more systematic orthography to phonology combining with the pretrained phonology to semantics system. Equally, a strong ODH position would also argue that readers of opaque orthographies such as logographic systems will depend on a direct route from orthography to semantics as the complexities of the orthographic to phonological mappings mean that learning such direct mappings no longer provides an advantage. The triangle model of reading (Seidenberg \& McClelland, 1989), implements a weaker version of ODH, in that it demonstrates how both direct and indirect routes are likely to be actively recruited during reading but to differing degrees depending on the systematicity of the mapping from orthography to phonology and to semantics (Harm \& Seidenberg, 2004; Plaut, McClelland, Seidenberg, \& Patterson, 1996).

Computational models have been developed to investigate division of labor within the reading system, but these have largely been limited to alphabetic systems (Harm \& Seidenberg, 2004; though see Chang, Welbourne, \& Lee, 2016; and Yang, Shu, McCandliss, \& Zevin, 2013). Harm and Seidenberg (2004) observed in their connectionist computational implementation of the triangle model that at earlier stages of training an advantage was observed for word comprehension tasks by processing via the indirect phonological pathway (so from orthography to phonology to semantics, compared with from orthography directly to semantics). However, this advantage reduced over the course of training such that some words could be processed only by the direct orthography to semantic route, and by the end of training approximately half the corpus could be read by either route. Thus, this computational study suggests that even for alphabetic (but deep) systems such as English both routes are likely to be recruited.

The question remains, as to whether the triangle model is an adequate framework to explain reading development regardless of the orthographic system, and if so what does it reveal about how orthographic systems affect processing within the reading system? The computational modeling literature is divided on this issue with distinct architectures often devised for alphabetic and logographic systems (e.g., Perfetti, Liu, \& Tan, 2005). Proponents of the dual route cascaded model (DRC), initially developed for processing the deep alphabetic system of English, for example, have suggested that because of the scale of structural differences between alphabetic, syllabic, and logographic systems the architecture they propose to support reading in alphabetic systems would not be applicable for syllabic or logographic systems (Coltheart et al., 2001). More recent computational modeling studies conducted by Yang, Zevin, Shu, McCandliss, and Li (2006, 2009, 2013) have made a substantial contribution to our understanding of the viability of the triangle model architecture to support Chinese reading and the effects of such an orthographic structure on processing. Yang, McCandliss, Shu, and Zevin (2009) focused on the emergent properties of networks trained purely on direct orthographic to phonological Chinese mappings. They observed that this single path is able to develop internal representations that take advantage of the small degree of systematicity carried in the logographic orthography regarding the phonological properties of the word in terms of the phonetic radicals. Furthermore, Yang et al. (2006, 2013) constructed a computational implementation of the triangle model similar to that of Harm and Seidenberg (2004) that was found to be able to support Chinese reading, and that in comparison to English it displayed a distinct developmental profile relying more heavily on orthographic to semantic mappings and learning these mappings more rapidly even than orthographic to phonological mappings. These models provide support for the position that reading, regardless of orthographic structure, can be supported by the same computational system operating over distributed representations of phonological, semantic, and orthographic information with orthographic systems affecting how those representations interact within the reading system.

Cognitive neuroscience studies have shown that, irrespective of the orthographic system, the neural architecture supporting reading across populations of different languages spans many of the same key brain regions (Bolger, Perfetti, \& Schneider, 2005; Das, Padakannaya, Pugh, \& Singh, 2011; Hervais-Adelman et al., 2019; Nakamura et al., 2012; Rueckl et al., 2015; Tan, Laird, Li, \& Fox, 2005; Taylor, Rastle, \& Davis, 2013) that is, the left lateralized brain networks shown to support spoken language processing (Devauchelle et al., 2009) that are largely in place from 2 months of age (Dehaene-Lambertz et al., 2010). There is, however, also neuroscientific evidence to support the existence of two distinct paths in the reading system (e.g., Cohen, Dehaene, Vinckier, Jobert, \& Montavont, 2008; Cummine et al., 2015; Dehaene, 2009; Jobard, Crivello, \& Tzourio-Mazoyer, 2003; Price, 2010; Richardson, Seghier, Leff, Thomas, \& Price, 2011; Taylor et al., 2013). Largely derived from studies conducted on alphabetic literate participants, neuroimaging data demonstrates that skilled reading recruits both a dorsal (orthography to phonology) path and a ventral (orthography to semantics) path and models of this dual stream have been implemented for alternative orthographies, such as Ueno \& Lambon Ralph's (2013) Japanese model.

Neuroimaging studies have also investigated changes to processing over the course of literacy training, although this research is again dominated by studies of alphabetic literates (but see Rueckl et al., 2015). Current data indicate a progression over the course of training in English literates from an initial bias toward use of the dorsal (orthographic to phonological) path to later dominance of the ventral (orthographic to semantic) path in pro- 
ficient readers (Chyl et al., 2018; Malins et al., 2018; Pugh et al., 2001; Shaywitz et al., 2002). These data have motivated contrasting interpretations. For example, Pugh et al. (2000, 2001) suggested that in proficient readers the dorsal path is only recruited for slow analytic mapping from orthography to phonology, while Levy et al. (2008, 2009) claimed that the dorsal path is primarily recruited for nonword reading, as in the DRC model (Coltheart et al., 2001). The latter perspective, however, seems unlikely given more recent data showing that the dorsal path is involved at both early and late stages of written word processing (Richardson et al., 2011).

Studies that have compared neural activation in literate populations that differ in the transparency of the orthography on which they were trained have revealed differences in activation of regions associated with orthography to phonology and orthography to semantics pathways. A study by Rueckl et al. (2015) examined cerebral blood flow (using functional magnetic resonance imaging, fMRI) as Spanish, English, Hebrew, and Chinese literates performed semantic categorization tasks given either spoken or written word stimuli. They observed greater overlap between speech and print processing networks in regions associated with phonological processing (supramarginal gyrus and supplementary motor area) in the most transparent system (Spanish) than the more opaque alphabetic and alphasyllabic/consonantal systems (English, Hebrew). In contrast, the less transparent orthographies of English and Hebrew generated greater overlap between regions activated by both speech and written words in regions associated with semantic processing (angular gyrus, middle temporal gyrus, and inferior temporal gyri). This pattern replicates earlier findings (Buchweitz, Mason, Hasegawa, \& Just, 2009; Kiyosawa, Itoh, Nakagawa, Kobayashi, \& Tamai, 1995; Paulesu et al., 2000; Thuy et al., 2004) that support the claim that more transparent systems are more reliant on the dorsal path, suggesting greater processing from orthographic to phonological mapping, while less transparent systems are more reliant on the ventral path, suggesting greater processing from orthographic to semantic mappings. However, it should be noted that in Rueckl et al. (2015) comparisons of cerebral blood flow between logographic (Chinese) literates and literates in the other more transparent systems (English, Spanish, and Hebrew) failed to support this trend, with only small difference observed in regions not typically associated with either semantic or phonological processing.

\section{Effects of Literacy on Representational Structure}

There is substantial debate regarding the impact of literacy acquisition on the language processing system, particularly the effect of orthography to phonology mappings influencing phonological representations (Araújo et al., 2019; Huettig \& Mishra, 2014; Morais \& Kolinsky, 2001; Petersson, Ingvar, \& Reis, 2009; Rastle, McCormick, Bayliss, \& Davis, 2011). Different orthographies may affect this restructuring. Two issues have hampered progress on this issue, first the difficulty of isolating any behavioral effects that result from implicit online processing as opposed to resulting from explicit metalinguistic operations, and second problems in isolating behavioral effects of orthographies from linguistic or sociocultural factors.

Learning to read an alphabetic language leads to increased performance in detecting and manipulating individual phonemes in speech (phonological awareness tasks), in both child (Alcock, Ngorosho, Deus, \& Jukes, 2010; De Jong \& Van der Leij, 1999; Hulme, Snowling, Caravolas, \& Carroll, 2005; Treiman \& Zukowski, 1991) and adult (Adrián, Alegria, \& Morais, 1995; Loureiro, Braga, Souza, Nunes Filho, Queiroz, \& Dellatolas, 2004; Morais, Cary, Alegria, \& Bertelson, 1979; Scliar-Cabral, Morais, Nepomuceno, \& Kolinsky, 1997) populations. Such tasks require participants to perform complex metalinguistic operations and, therefore, may change the thinking about the speech rather than change the perception of speech (e.g., see Mitterer \& Reinisch, 2015). There is evidence from computational models (Harm \& Seidenberg, 1999; Smith, Monaghan, \& Huettig, 2014a, 2014b); however, that supports the notion that changes in processing to finer-grained phonological processing occur during online speech processing as a consequence of alphabetic orthography literacy (see also Hoonhorst et al., 2011; Huettig \& Mishra, 2014; Huettig, Singh, \& Mishra, 2011; Reis \& Castro-Caldas, 1997; Serniclaes, Ventura, Morais, \& Kolinsky, 2005).

Three theoretical models have been proposed for how learning orthographic mappings may affect phonological processing. First, it could be that orthographic representations are activated online during speech processing, and interconnections to and from orthography then influence phonological processing (online activation hypothesis; e.g., Ziegler \& Ferrand, 1998), consistent with Price and Devlin's (2011) interactive account of ventral processing in reading. Second, learning to map between orthographic and phonological representations may lead to a restructuring of phonological processing (e.g., Muneaux \& Ziegler, 2004; Ziegler \& Goswami, 2005). Third, it could be that literacy results in both online activation of orthography and phonological restructuring (e.g., Dehaene et al., 2010). Dehaene et al. (2010) used fMRI to compare brain activity in illiterate and alphabetic literate populations when processing spoken words. In literate, but not illiterate, populations they observed activation of brain regions associated with orthographic processing (left occipital temporal cortex), as well as a near doubling of activation in the planum temporale, a region associated with phonological processing (see also Monzalvo \& Dehaene-Lambertz, 2013). This supports the hypothesis that orthographic representations are activated online and that there are changes to phonological representations as a consequence of literacy training. However, recent fMRI studies (HervaisAdelman et al., 2019; Skeide et al., 2017) compared neural activation in an illiterate population, an alphasyllabic literate population and a late alphasyllabic literate population, with control over the cultural backgrounds of these groups, over the course of exposure to literacy training. They found no evidence for differences in activation in auditory cortex when listening to speech in either cross-sectional or longitudinal comparisons, although differences could be observed in auditory cortex when processing written text. Therefore, this suggests that either restructuring of auditory processing regions does not occur or that it does not lead to differences in the levels of activation in such regions when processing continuous speech. There is also additional complexity in making inferences from such imaging data as the temporal resolution of fMRI may not be reflecting only online language processing of the few hundred milliseconds of the spoken word but also potentially longer-term side effects not critically involved in early stages of phonological encoding. Using electroencephalogram (EEG), which offers 
greater temporal resolution, Perre, Pattamadilok, Montant, and Ziegler (2009) demonstrated that orthographic effects in lexical decision were localized to phonological processing areas (left BA40), and Pattamadilok et al. (2010), using transcranial magnetic stimulation (TMS), found that orthographic consistency effects were eliminated when phonological regions were manipulated; yet, no effect was observed when TMS was applied to orthographic processing regions. These studies are consistent with the theory that phonological representations are restructured as a consequence of literacy training. Existing cognitive neuroscientific data are far from conclusive; yet, suggest that exposure to alphabetic literacy training can affect the structure of phonological processing regions involved in online speech processing. This raises the question as to whether development of literacy in other orthographic systems exerts the same effects on phonological processing. Psycholinguistic grain size theory (Ziegler \& Goswami, 2005) predicts that the nature of the correspondences between graphemes and phonological units for a given orthographic system will define the impact of literacy training on phonological processing. On this basis, alphabetic literates will exhibit finer grain effects on phonological processing than logographic literates because of the regular systematic relations between individual graphemes and phonemes in alphabetic systems, hence the phonological restructuring proposed to result in phoneme awareness abilities and finergrained processing of speech in visual world processing tasks is likely to be observed primarily as a consequence of literacy for alphabetic orthographies. Indeed, it is a possibility that differences in transparency between Portuguese (the participants in Dehaene et al., 2010, were from Brazil and Portugal) and Devanagari (the participants in Hervais-Adelman et al., 2019, were from Uttar Pradesh, India) explain the different fMRI findings in their studies.

It is becoming more difficult to test literate populations that are not exposed to alphabetic systems because of the widespread use of English, and also because of the increasing simultaneous use of alphabetical or alphasyllabic orthographic systems alongside deeper orthographies, for example, the increasing use of Pinyin accompanying traditional writing for Mandarin Chinese (Cheung \& Ng, 2003). There is, however, some evidence to support the predictions of psycholinguistic grain size theory and the predictions of the computational work described above. Brennan, Cao, Pedroarena-Leal, McNorgan, and Booth (2013), for example, used fMRI to compare the brain activity of Chinese and English literate adults and children when performing rhyme judgment tasks. They observed differences over the course of development in phonological processing regions (superior temporal gyrus) only in comparisons between English speaking adults and children. An earlier behavioral study by Read, Zhang, Nie, and Ding (1986) tested adults who were literate either only in logographic Chinese or in both alphabetic and logographic scripts. Their results showed that only the group literate in both the alphabetic and logographic scripts were able to add or delete individual consonants in spoken Chinese words. In a similar study, De Gelder and Vroomen (1992) compared a group of Dutch literates, a group of Chinese literates and a group literate in both Dutch and Chinese on their ability to distinguish between /ba/ and /da/ drawn from a nine step continuum. They showed that alphabetic literates (Dutch) and literates of two scripts (Chinese [logographic] and Dutch [alphabetic]) displayed sharper phonological boundary precision than logographic (Chinese) literates. Further, Cheung and Chen (2004) examined participants' ability to perform sound matching and primed shadowing tasks, while Shu, Peng, and McBride-Chang (2008) tested phoneme onset awareness, and both demonstrated that performance on these phonological awareness tasks in Chinese literates coincided with exposure to Pinyin tuition. However, in contrast to such findings, Kidd, Shum, Ho, and Au (2015) showed that logographic literates can show sensitivity to phonological structure in speech gating and nonword repetition tasks; thus, any effect of orthography appears to be one of degree rather than a qualitative change in processing, though it was unclear the extent to which these participants were exposed to Pinyin.

Thus, there is substantial theoretical and empirical support in the literature for an effect of orthographic transparency on phonological processing. However, questions remain as to the extent to which observed effects result from a restructuring of the phonological processing regions engaged during online speech processing or from coactivation of orthographic codes.

In summary, then, the key behavioral phenomena to address for a model implementing a variety of orthographies in reading are:

- Orthographic transparency aids phonological decoding, while effects of orthographic transparency on reading comprehension are less clear (see Effects on acquisition);

- Orthographic transparency interacts with exposure to affect differences in processing over the course of reading development such that, at least at earlier stages of reading development, more transparent systems bias processing along dorsal pathways associated with orthographic to phonological mappings (see Effects on processing); and

- Effects of orthographic transparency on phonological processing, with increased transparency generating stronger activation of phonological representations for both naming and comprehension tasks (Effects of literacy on representational structure)

Before presenting the computational model of orthographic systems, we first investigate the extent to which there is dependence between orthographic system and language properties. It has been argued that "languages get the writing system they deserve" (Seidenberg, 2011; see also Frost, 2012; Rogers, 1995), which expresses the idea that observed variation in orthographic complexity is driven by a requirement to maintain efficient reading compression given variation in the complexity of spoken forms in the language. Hence, the pairing of a language and orthographic system is proposed to not be arbitrary. However, the level to which such pairings are arbitrary, and which linguistic factors determine the orthographic selection, have remained, for decades, hotly debated issues (see Rogers, 1995, for review), but there is a lack of typological studies testing these claims in the literature. In the following section, we address this lack of typological analysis. If orthographic systems are closely related to phonological properties of the language, then this means that an endeavor to examine the cognitive implications of orthographic systems independent of language variation is in vain. However, if a variety of orthographic systems are shown to apply across variation in phonological structure of languages, then this means that it is a prerogative to 
examine how orthographic system affects reading when language structure is kept constant.

\section{The Influence of Language Structure on Orthography: Do Spoken Languages Get the Orthographic Systems They Deserve?}

Earlier views, originating predominantly from countries in which alphabetic systems are dominant, conceived of the role of orthography as primarily for coding phonological forms of a language. Thus, the "best" orthography was one in which the phonology was richly coded (conveniently being the alphabetic systems already in use). Over recent decades, however, a growing acknowledgment has emerged of the role of other properties of language in determining orthographic structure (e.g., phonotactics, morphology, and syntax), and the fundamental role played by orthography in facilitating mapping onto the semantic structure of a language (Frost, 2012; Rogers, 1995; Venetzky, 1970). For example, irregularities in correspondences between orthography and phonology in English have long been given as evidence for the language's underlying representation being prioritized over its phonological form (Chomsky, 1970; Klima, 1972; although also see Sampson, 1985 and Francis, 1970).

Rogers (1995) argues that languages containing many fused forms favor an alphabetic system, while languages that contain many homophones favor logographic systems, because they permit heterographs (same phonology, different spelling and meaning). Seidenberg (2011) identified the complexity of the inflectional morphology as a determining factor arguing that shallow orthographies are consistently paired with languages with more complex inflectional morphologies. A number of scholars have ventured beyond identifying individual factors to posit that each orthographic system represents an optimal solution to the unique set of constraints the given language poses (Frost, 2012; Olson, 1988; see Rogers, 1995 for discussion). Frost supports this view by providing five examples: the extensive homophony within Chinese is best accommodated by a logographic system; Japanese, a multisyllabic morae-timed language with a relatively small syllabary, is ideally suited to a syllabic system; the agglutinative nature of Finnish predisposes it to its extremely transparent alphabetic orthography; the large and complex English syllabary gives rise to its deep alphabetic system; and the highly constraining root-derived language Hebrew results in its consonantal orthography.

Although these specific examples arguing for tight relationships between orthographic system and language structure seem compelling, pairings of languages with orthographic systems might also be affected by historical, geographical, and/or cultural factors (Behme, 2012; Beveridge \& Bak, 2012; Norris \& Kinoshita, 2012; Rogers, 1995; Seidenberg, 2011; Share, 2012). For example, a change in Turkish from Arabic to Latin script in 1928, or a change in Romanian from Cyrillic to Latin script (Ghetie, 1978; see Beveridge \& Bak, 2012 for discussion) were driven by political decisions. Further, as Share (2012) identifies, of the approximately 500 African languages to possess a written form the vast majority use a European Romanized alphabet largely devised by missionaries who assumed such scripts to be inherently superior (see Gelb, 1952; Havelock, 1982). Historical reasons appear to be a main determinant with which orthographic system a given language is written. All Indo-Aryan languages in Northern India are written in scripts that evolved from Brahmi script. Tamil, like all South Indian Dravidian scripts, for purely historical reasons also derives from Brahmi script even though Dravidian and Indo-Aryan languages are unrelated (the curved forms of the South Indian scripts originate from the constraint that they used to be written on palm leaves that split if a straight line is drawn on them). Additionally, there are many examples of a single language being paired with multiple scripts (e.g., Arabic [Abjad], Cyrillic [Alphasyllabic] in Kazakh; Shahmukhi [Consonantal], Gurmukhi [Alphasyllabic] and Devanagari [Alphasyllabic] in Punjabi); therefore, although it is feasible one system may be better suited, it is apparent that there are multiple cases where different categories of orthographic system can function effectively for the same language, sometimes simultaneously.

\section{A Typological Analysis of Phonological Features of Orthographic Systems}

To examine the linguistic space occupied by different categories of orthographic system we examined five phonological properties that have been proposed to bias a language toward a given orthographic system: The size of a language's consonant inventory (Maddieson, 2013a), the number of vowel contrasts in a language's inventory (Maddieson, 2013b), the ratio of consonants to vowels within a language (Maddieson, 2013c), a measure of syllable structure capturing the phonotactic constraints within which consonants and vowels can be combined to form syllables (Maddieson, 2013d), and complexity of tonal structure (Maddieson, 2013e). ${ }^{2}$ For each language in the World Atlas of Language Structures (Dryer \& Haspelmath, 2013) for which all the above measures were available we cross-referenced with the Ethnologue: Languages of the world database (Simons \& Fennig, 2018) to obtain the script in which each given language is frequently written. Scripts were then assigned to one of five categories of orthographic system (alphabetic, abugida, abjad, syllabic, and logo-syllabic) based on definitions retrieved from ScriptSource (http://www.scriptsource.org). ${ }^{3}$ Thirty-six languages were recorded as encoded in more than one orthographic system. In such cases if it was unclear in which system the language was most frequently encoded, then the globally least common system was assigned. ${ }^{4}$ This method was used in the case of 12 abjad systems, 14 abugida systems, three syllabic systems, and seven logosyllabic systems. This resulted in a total of 357 languages each with their attributed orthographic system(s), comprising 287 alphabetic, 35 abugida alphasyllabic, 21 abjad (consonantal), four syllabary, and 10 logo-syllabary languages.

\footnotetext{
${ }^{2}$ As reviewed above, morphological properties have also been proposed to significantly influence selection of orthographic systems, our analysis focuses on phonological language properties. This is because of the greater availability of comprehensive descriptions of phonological properties, but not morphological features, in databases for cross-referencing with orthographic systems. Future studies analyzing rich morphological measurements will be a valuable line of further investigation.

${ }^{3}$ Note that in the Scriptsource database, logographic systems are classified as logo-syllabic.

${ }^{4}$ Analyses were also performed on the subset of languages for which a more frequent system was identifiable. Results of these analyses were similar to those reported for the full data set. See online Supplemental Materials Section E for further details of these additional analyses.
} 
Consonant inventory (Maddieson, 2013a). Size of a languages consonant inventory is categorized at one of five levels in the World Atlas of Language Structures (WALs) database. Languages with an inventory of $22 \pm 3$ consonants are categorized as average, those with $6-14$ as small, $15-18$ as moderately small, $26-33$ as moderately large, and more than 34 as large. Though analyses with a more fine-grained characterization of phonological properties (such as exact consonant inventory size) would provide greater precision in the analyses, this information is unfortunately not available within the WALs database. Figure 1 shows the proportion of languages of each orthographic system distributed across varying consonant inventory sizes. The Figure shows that alphabetic, abugida, and abjad orthographic systems are in use for languages with both small (or moderately small) and large consonant inventories, syllabic orthographic systems are in use for languages with small and average consonant inventories but not large consonant inventories, and logo-syllabic orthographic systems are in use for average and large consonant inventories, but not small consonant inventories. However, the distribution was not significantly different than chance, $\chi^{2}(16)=22.418, p=.130$ (though the categories contain small values, so the $\chi^{2}$ value should be interpreted with caution).

Vowel quality inventory (Maddieson, 2013b). Languages with five or six vowels were classed as average, four or less were categorized as small, and seven or more classed as large. Figure 2 shows the proportion of languages for each orthographic system by vowel quality inventory. The distribution of orthographic systems by vowel quality inventory size was not significantly different than chance, $\chi^{2}(6)=11.429, p=179$. As with the results from consonant inventory size, this indicates that vowel quality inventories of all sizes are represented across the orthographic systems.

Consonant-vowel ratio (Maddieson, 2013c). Consonant-vowel ratio was determined by dividing the number of consonants in a language by the number of vowels. A value of 2.00 or below was classed as a low ratio, 2.00-2.75 was categorized as moderately low, 2.75-4.5 classed as average, 4.5-6.5 as moderately high and greater than 6.5 as high. Figure 3 shows that though syllabic orthographic systems tend not to be used for languages with high consonant-vowel ratios, but the distribution was not significantly different than chance, $\chi^{2}(16)=14.274, p=.578$.

Syllable structure (Maddieson, 2013d). This measure reflects the variability with which the consonants and vowels of a

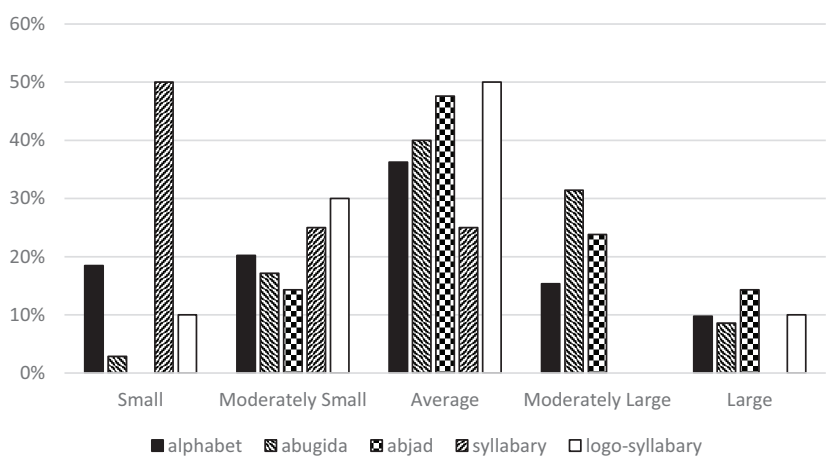

Figure 1. Proportion of languages with each type of script for each consonant inventory size.

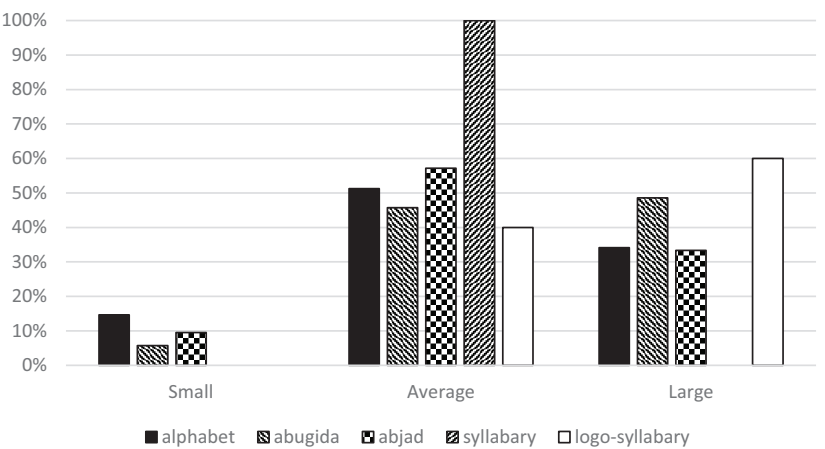

Figure 2. Proportion of languages by writing system for each vowel quality inventory size.

given language can be combined to form syllables. Languages that only permit syllables of the form $\mathrm{C}(\mathrm{V})$ are characterized as possessing simple syllable structure, languages who permit syllables of a complexity up to CCVC were classed as moderately complex, while those that allowed syllables of greater complexity were categorized as having complex syllable structure. Figure 4 indicates that abjad systems only represent languages that possess moderately complex or complex syllable structure, and alphabetic languages are more likely than other orthographic systems to represent simple syllable structure languages, and the distribution was significant, $\chi^{2}(6)=38.227$, $p<.001$.

Tone (Maddieson, 2013e). Languages were grouped into three categories: No tone systems, a simple tone system for languages with only a two-way basic contrast, and complex tone systems. Figure 5 indicates that logographic systems tend to represent languages that possess complex tonal structure, $\chi^{2}(6)=26.018, p=.001$.

Geographical location. Languages were classified according to six continental regions. Figure 6 shows a strong correspondence between orthographic system and location with abjad, abugida, and logographic languages tending to be located in Eurasia and alphabetic languages distributed more widely across the world, $\chi^{2}(20)=94.624, p<.001$.

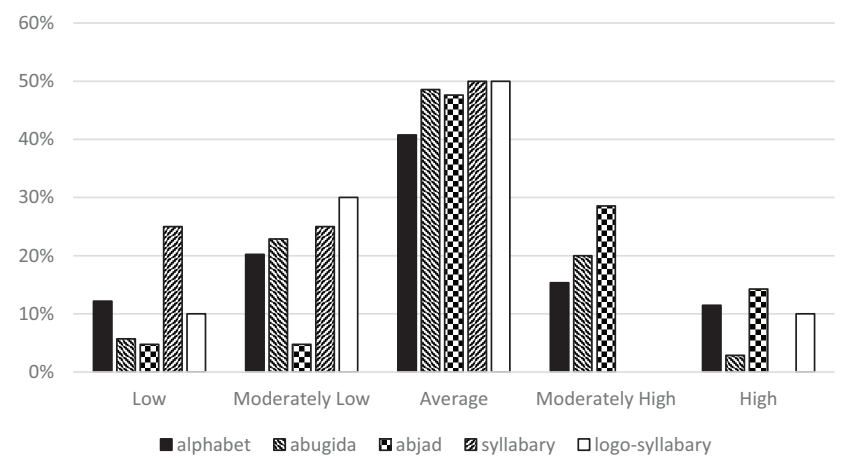

Figure 3. Proportion of languages by writing system for each category of consonant-vowel ratio. 


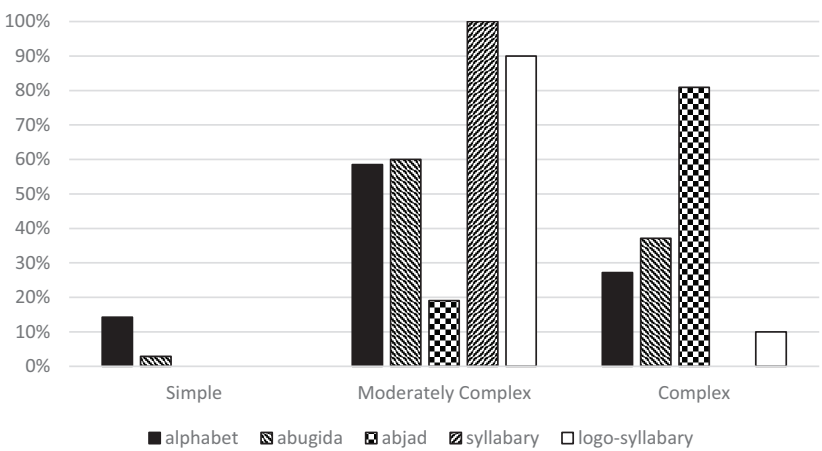

Figure 4. Proportion of languages by writing system for each category of syllable structure.

\section{Combinations of Phonological Features and Geographical Location}

To determine the relations between multiple phonological properties of the language and the orthographic system it uses and to pitch this against predictions from the geographical proximity of languages, we employed the multinom function in the nnet package (Venables \& Ripley, 2002) in R to perform multinomial logistic regression. To determine a more fine-grained assessment of the role of geographical location on orthographic system, geographical distance between each language and its closest neighbor for each category of orthographic system was calculated, generating five additional measures (distance to nearest alphabetic language, distance to nearest abugida language, distance to nearest abjad language, distance to nearest syllabic language, and distance to nearest logo-syllabic language). This distance measure was derived from the latitude and longitude attributed to each language within the WALs (Dryer \& Haspelmath, 2013) database using the geodist function in the $\mathrm{R}$ ( $\mathrm{R}$ Core Team, 2018) package gmt (Magnusson, 2017). All distances were log transformed before analysis.

These analyses predicted the orthographic system (alphabetic, abugida, abjad, syllabic, and logosyllabic) given the phonological structure of the language and/or its geographical proximity to other orthographic systems. Alphabetic systems were implemented as the reference level for the orthographic system.

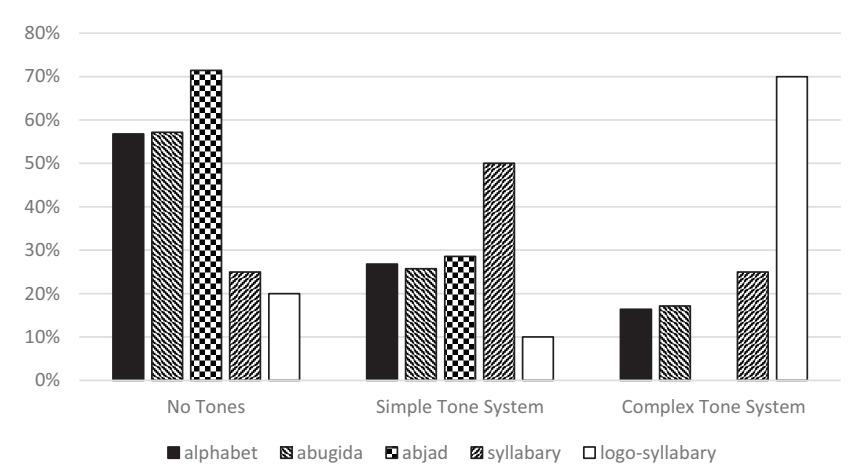

Figure 5. Proportion of languages for each writing system by tone system.

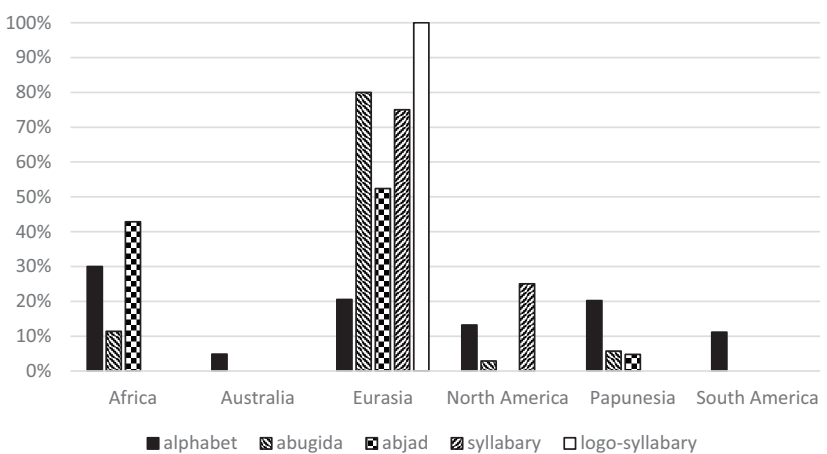

Figure 6. Proportion of languages by writing system in each geographical region.

Combined phonological structure analysis. The first model containing only measures of phonological structure (Akaike's Information Criterion, AIC $=495.54$ ) indicated that a larger vowel quality inventory is more probable in abugida systems than alphabetic systems $(\beta=0.617, z=2.05, p=.041)$, increased syllabic complexity is more probable in abjad systems than alphabetic systems $(\beta=2.11, z=3.54, p<.001)$, while increased tone complexity is more likely in logo-syllabic systems than alphabetic systems $(\beta=1.48, z=2.96, p=.003)$. There was also a marginal difference of consonant inventory size for abugida $(\beta=0.326, z=$ $1.90, p=.058)$ and syllabic $(\beta=-0.987, z=-1.717, p=.086)$ systems relative to alphabetic systems. The performance of this model to predict the correct orthographic system was tested using leave-one-out cross-validation. This was performed by fitting the model on all bar one of the available languages and then testing whether the fitted model was able to correctly predict the system of the language not included in the training set. Figure 7 displays the proportion of languages in each category of orthographic system that were correctly assigned. The phonological structure only model correctly assigned all alphabetic languages to the alphabetic category but was unable to assign any other system correctly.

A second model that also included second-order interactions between measures of phonological structure significantly improved the model $($ AIC $=531.72)$. The model showed a significantly greater level of syllabic complexity in abjad languages relative to alphabetic languages $(\beta=2.32, z=2.87, p=.004)$ and greater tone complexity in logo-syllabic systems relative to alphabetic systems $(\beta=2.22, z=2.19, p=.029)$. Finally, there was a marginal interaction between the size of the consonant inventory and vowel inventory when comparing abugida systems to alphabetic systems $(\beta=0.471, z=1.71, p=.088)$. Leave-one-out cross-validation was again performed, this model with interactions included performed at a similar level to the previous model predicting $99 \%$ of alphabetic systems however performance on nonalphabetic systems was very weak identifying no other system correctly other than one abugida language.

Geographical proximity analysis. A model containing only measures of geographical proximity was implemented, and provided a better fit compared with the models containing the combined phonological features $(\mathrm{AIC}=303.1)$. The model showed differences in the geographical proximity to alphabetic systems for 


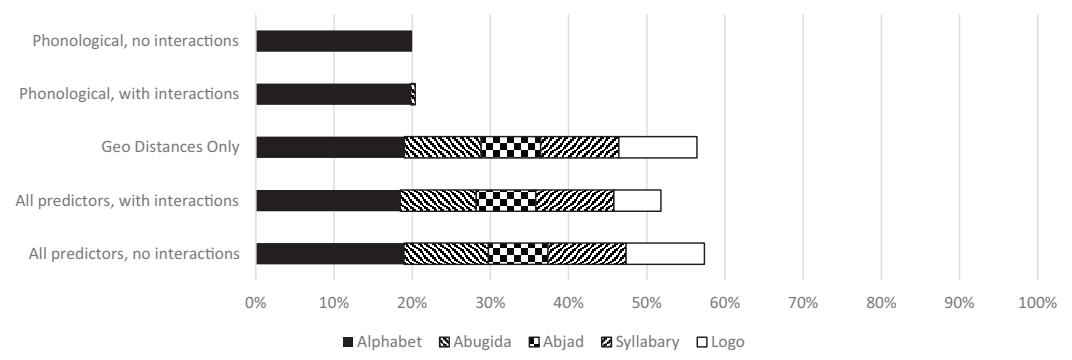

Figure 7. Proportion of writing systems correctly predicted from phonological structure and/or geographic distance measures using multinomial logistic regression and leave-one-out cross validation (note maximum value for any one writing system is $20 \%$ ).

abjad $(\beta=1.92, z=4.78, p<.001)$, abugida $(\beta=0.630, z=$ $2.21, p=.027)$, and logo-syllabic $(\beta=2.56, z=2.91, p=.004)$ languages relative to alphabetic. Abugida $(\beta=-1.17, z=-2.93$, $p=.003), \log 0$-syllabic $(\beta=-1.67, z=-2.58, p=.010)$, and syllabic $(\beta=118.7, z=27.2, p<.001)$ all differed in their proximity to syllabic systems relative to alphabetic. Abugida $(\beta=-1.02, z=-4.35, p<.001)$ and syllabic $(\beta=-10.1$, $z=-24.3, p<.001)$ languages also differed in their proximity to abugida systems relative to alphabetic languages. Abjad $(\beta=-1.71, z=-5.11, p<.001)$ and abugida $(\beta=-0.714$, $z=-2.34, p=.019)$ languages also differed in their proximity to abjad systems relative to alphabetic languages. Further, logosyllabic $(\beta=-2.03, z=-3.10, p=.002)$ and syllabic $(\beta=-51.1, z=-24.3, p<.001)$ systems differed in their proximity to logo-syllabic systems relative to alphabetic languages. Leave-one-out cross-validation performance exceeded that of the model including all phonological predictors. A model containing only geographical proximity information was able to identify $95 \%$ of alphabetic systems, $49 \%$ of abugida systems, $38 \%$ of abjad systems, $50 \%$ of syllabic systems, and $50 \%$ of logographic systems.

Combined geographical proximity and phonological structure analysis. A fourth model included all measures of phonological structure with second-order interactions, in addition to all measures of geographical proximity. This model outperformed the phonological structure only models but not the geographical proximity only model (AIC $=350.30)$. Leave-one-out cross-validation demonstrated that such a model was able to identify $92 \%$ of alphabetic systems, $49 \%$ of abugida systems, $38 \%$ of abjad systems, $50 \%$ of syllabic systems, and $30 \%$ of logographic systems.

A fifth model including all measures of phonological structure and geographical proximity; yet, no interactions, again did not improve on the model containing only geographical proximity $(\mathrm{AIC}=307.52)$. Leave-one-out cross-validation showed a small improvement on the model that included interactions, the reduced model identified $95 \%$ of alphabetic systems, $54 \%$ of abugida systems, $38 \%$ of abjad systems, $50 \%$ of syllabic systems, and $50 \%$ of logo-syllabic systems.

\section{Summary of Orthographic System by Phonological and Geographic Features}

This quantitative analysis suggests that although certain characteristics of a language may favor a particular orthographic system, there appear to be many exceptions in which instead historical, geographical, and/or cultural factors have determined the orthographic system in use. ${ }^{5}$ Indeed, once geographical proximity is taken into account, the language structure features do not predict the orthographic system significantly. Thus, the language does not (always) get the orthographic system it deserves, and so exploring the role of orthographic systems in affecting processing within the reading system, when controlling the phonology and meaning space of the language, will provide insight into a broad range of literate cultures. We do, however, return to this issue, in section Examining the Effects of Orthographic Transparency: Disyllabic Simulations, when we provide further computational investigations into the effect of adjusting the phonology of the language and the cognitive consequences of different orthographic systems.

\section{Modeling the Effects of Orthographic Systems}

The goals of this study are as follows. First, we determined the range of the triangle model as a universal architecture able to implement reading across each of the world's major orthographic systems. Dual path models have been questioned as to their relevance beyond their origins in the alphabetic literature (e.g., Share, 2008); however, previous studies have demonstrated the triangle model's ability to support logographic and alphabetic systems. In this study we extend the model with implementations of syllabic, alphasyllabic, and consonantal systems in addition to alphabetic and logographic systems.

Second, after assessing the triangle model's adequacy in simulating reading across orthographic systems, we investigate the effect of different orthographic systems on the way in which the reading system develops as a consequence of literacy. Previous neuroimaging and computational modeling studies, as discussed in sections Effects on acquisition and Effects on processing, suggest that although literates trained on differing orthographic systems activate very similar neural populations when reading, transparency affects the nature of the computations performed and more specifically it likely alters the division of labor across processing

\footnotetext{
${ }^{5}$ Given multinomial models can be biased to fit the largest category, weighted analyses were also performed with each orthographic system contributing equally to the categorization. Results of these weighted multinomial models were similar to those of unweighted model, further supporting above conclusions and can be found in online Supplemental Materials Section E.
} 
pathways. Further, such studies also suggest that the nature of such differences in processing are likely to change over the course of literacy training. Previous studies have, however, struggled to isolate the specific contribution of orthographic transparency because of covarying linguistic or socioeconomic factors. We hypothesized, based on the available data, that varying systematicity between orthography and phonology and orthography and semantics would result in distinct patterns of division of labor along pathways between orthographic, phonological, and semantic representations, and that these are likely to vary during the course of reading development (Chang et al., 2019; Harm \& Seidenberg, 2004). Specifically, orthographic transparency will, at least during early stages of literacy training, increase reliance on the direct orthographic to phonological mapping (dorsal) path, while increased semantic transparency would increase reliance on the direct orthographic to semantic mapping (ventral) path. Implementation in a computational model allows us to isolate the extent to which any observed effects could result purely from differences in orthographic structure, without reference to broader cultural, social, or educational differences.

Third, as discussed in section Effects of literacy on representational structure previous neuroimaging and behavioral studies have demonstrated effects of orthographic structure on phonological processing. However, the extent to which these effects are because of a restructuring of phonological processing regions and/or activation (online or offline) of orthographic codes remains hotly debated (Dehaene et al., 2010; Perre et al., 2009; Huettig et al., 2015; Mitterer \& Reinisch, 2015). Thus, we tested whether training on orthographic mappings generate restructuring effects in the triangle model and whether the nature of restructuring is influenced by orthographic transparency. We also tested whether the triangle model predicts distinct effects of orthography on phonological processing in the presence or absence of orthographic activation. We hypothesized that the model trained on an alphabetic orthography would demonstrate finer-grained phonological processing than the model trained on logographic systems. Alphasyllabic orthographies were predicted to lie somewhere in between.

To examine each of these issues we trained a connectionist neural network model based on Harm and Seidenberg's (2004) implementation of the triangle model of reading, but using artificial corpora consisting of orthographic, phonological, and semantic representations. Constructing artificial corpora ensures that relations both within and between representational domains are controlled (see Hirshorn and Fiez (2014) and Plaut et al. (1996) for similar justification). Consequently, controlling semantic and phonological representations and mappings across orthographic systems ensures any observed differences in behavior or processing are driven by differences in the orthographic structure.

The orthographies selected for modeling represented each of seven orthographic structures: shallow alphabetic, deep alphabetic, alphasyllabic, consonantal, syllabic, logographic semantically opaque, and logographic semantically transparent. Critically, identical semantic and phonological representations and mappings were used across all orthographic systems, and all training and testing parameters of the model were controlled to ensure that observed behavioral effects of the model stemmed only from effects of the orthographic representations and did not reflect aspects of phonological and semantic structure. Simulations were run separately on a monosyllabic language with a complex syllabary (see Examining the Effects of Orthographic Transparency: Mono-Syllabic Simulations) and a disyllabic language with a simple syllabary (see Examining the Effects of Orthographic Transparency: Disyllabic Simulations) to test the generalizability of model behavior across languages structures. Syllabic complexity was selected as the dimension of manipulation as it is frequently cited as a biasing factor in determining orthographic system, and our survey of orthographic systems supported such claims.

This close control and comparison of models of multiple orthographies enable a test of theoretical and neuroscientific accounts of division of learning rates of reading fluency and reading comprehension affected by orthography, and the extent to which the architecture of the reading system in terms of direct and indirect routes and division of labor can explain apparent behavioral distinctions in reading performance.

\section{Model Design}

Architecture. Model architecture (see Figure 8) was closely matched to Harm and Seidenberg's (2004) implementation of the triangle model of reading, differing only in terms of the number of units within each of the layers. The illiterate/preliterate network consisted of an interconnected phonological and semantic layer, to simulate spoken language processing. The semantic layer comprised 150 units. These units were fully connected to a set of 25 semantic clean-up units, which were fully connected back to the semantic layer. These clean-up units were included to ensure that stable semantic representations could be formed in the semantic

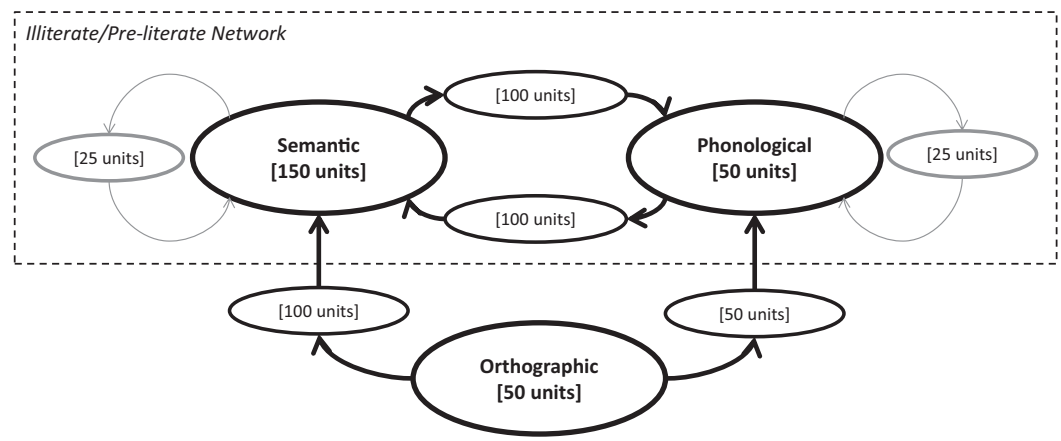

Figure 8. Model architecture. 
layer. The semantic layer was fully connected to a hidden layer of 100 units that was in turn fully connected to a phonological layer consisting of 50 units. The phonological layer was also connected to a set of 25 phonological clean-up units that were connected back to the phonological layer. The phonological layer was also connected to the semantic layer via another hidden layer consisting of 100 units. The reading model built on the preliterate/illiterate network with the addition of an interconnected orthographic layer. The orthographic layer consisted of 50 units, which was connected to the phonological and semantic layers via two hidden layers of 50 and 100 units, respectively. Numbers of units in the hidden layers were determined by pilot studies to determine the minimum number of units required to acquire accurate mappings between representations, with the exception that in all pilot simulations the number of hidden units in the orthography to phonology path was half that of the number in the hidden layer connecting orthography to semantics, which reflected the architectural constraint implemented in Harm and Seidenberg (2004). A bias unit was fully connected to each layer within the network. Simulation files and data are archived at http://osf.io/vudhk.

Representations of words. Artificial corpora of words were constructed consisting of 500 unique items. Each item represented a unique monosyllabic word and was assigned a phonological, semantic, and orthographic form. For each item, the semantic representation was unique, but there was some overlap between the phonological and orthographic representations, to simulate homophony in the corpus. Homophones were included to ensure a distinction between syllabic and logographic systems at the monosyllabic level (see Orthographic representations). ${ }^{6}$ Eight different artificial corpora were generated for each of the seven orthographic systems to be simulated.

Semantic representations. Semantic representations were encoded by a 150 unit binary feature vector, with each unit representing a distinct semantic feature (Dilkina, McClelland, \& Plaut, 2008, 2010; O’Connor, Cree, \& McRae, 2009; Rogers et al., 2004). Ten prototype patterns similar to those used in Dilkina et al. $(2008,2010)$ were used to construct an artificial semantic taxonomy; consisting of two high-level semantic categories, each with five subcategories (see Figure 9). Fifty items were generated per sub category (per prototype pattern). Each prototype pattern vector was divided into 10 subsets of features, each containing 15 units, with each feature within a given subset more likely to be shared between items within the same semantic category. For each item, the 15 features assigned to its given subcategory were $\mathrm{p}$ (active) $=$ 0.267 , the remaining 60 features assigned to its given higher level category were $\mathrm{p}$ (active) $=0.133$, and the remaining 75 features assigned to the higher level semantic category to which it was not assigned were $\mathrm{p}$ (active) $=0.040$.

Phonological representations. Phonological representations were five phonemes in length and of the form CCVCC. ${ }^{7}$ The phonological layer consisted of five phoneme slots, organized CCVCC, with each slot 10 units in length. A phoneme inventory consisting of five vowels and 10 consonants was constructed. Each phoneme was encoded by a unique 10 unit phonological feature vector with $\mathrm{p}($ active $)=0.5$. Phonemes were pseudo randomly sampled from the phoneme inventory to construct words while ensuring across all words each phoneme was used an equal number of times in each phoneme slot (ignoring homophones). Four hundred and 50 distinct phonological representations were con- structed, and then a further 25 , distinct from the 450 , were included twice, mapping to different orthographic and semantic representations, to simulate presence of 25 homophones in the language.

The phonological representations of 50 nonwords were also constructed by randomly sampling phonemes from the phoneme inventory. Nonwords were not used in training but used to test the model posttraining to examine the model's ability to generalize to novel forms for alphabetic, alphasyllabic, and consonantal orthographies (see online Supplemental Materials Section A).

Orthographic representations. Seven forms of orthographic structure were implemented, based on the descriptions of different orthographic systems provided in Comrie (2013). All orthographic representations consisted of a 50 unit binary feature vector where each feature had $\mathrm{p}$ (active) $=0.5$. For all orthographic types, apart from the logographic semantic transparent system, the mapping between orthography and semantics was arbitrary-with a random linking from orthographic and phonological representations to the semantics. This was used to reflect the quasi-arbitrary mapping between spoken and meaning forms (Monaghan, Shillcock, Christiansen, \& Kirby, 2014), and between written and meaning forms (Aronoff, Berg, \& Heyer, 2016; Ulicheva, Harvey, Aronoff, \& Rastle, 2020).

Alphabetic shallow. In alphabetic systems the basic unit of representation is the phoneme. The orthographic layer was defined in terms of five letter slots, organized in a CCVCC structure to match that of the phonology, where each slot contained 10 units. All words consisted of five letters taken from an alphabet of 15 letters, where five letters represented vowels and 10 represented consonants.

In the shallow alphabetic system there was perfect correspondence between occurrence of a letter and a phoneme. Each letter within the alphabet was assigned to a phoneme. Orthographic representations were constructed using this regular, transparent

\footnotetext{
${ }^{6}$ Homophones refer to words with the same phonology, but different semantics. These can be heterographs (where the orthography is different) or homonyms (where the orthography is the same). For alphabetic, alphasyllabic, and syllabic orthographies, the implementation of homophones in our simulations are as homonyms. For the consonantal orthography, the simulation contains the same set of homonyms but in addition a number of heteronyms (same orthography, different phonology, and semantics) because words with the same consonants but different vowels are written in the same way. Finally, for logographic orthographies, the simulations contain heterographs (same phonology, different orthography, and semantics). Prevalence of homophones and homographs has been proposed to vary across orthographic systems (e.g., Frost, 2012), but we were unable to find any databases to determine this, and so we focus in the current article on investigating the effect of different orthographies when the set of phonological and semantic representations are kept constant across orthographic systems.

${ }^{7}$ Languages vary in terms of whether or not they contain consonant clusters, and also regarding word length in terms of how representative a monosyllabic subset of the language would be. In this respect, we are abstracting away from the properties of particular languages, but incorporating sufficient complexity within the phonological forms to permit overlap and distinctiveness in the representations, reminiscent of the patterns found across a wide set of languages. Fixing phonological characteristics across orthographic types enables us to keep the semantic and phonological representations the same, to isolate the contribution of variation in the mappings from orthographic representations to reading performance and language processing. However, Simulation 4 varies the phonological characteristics to explore the influence of word length and syllabic complexity on orthographic types.
} 


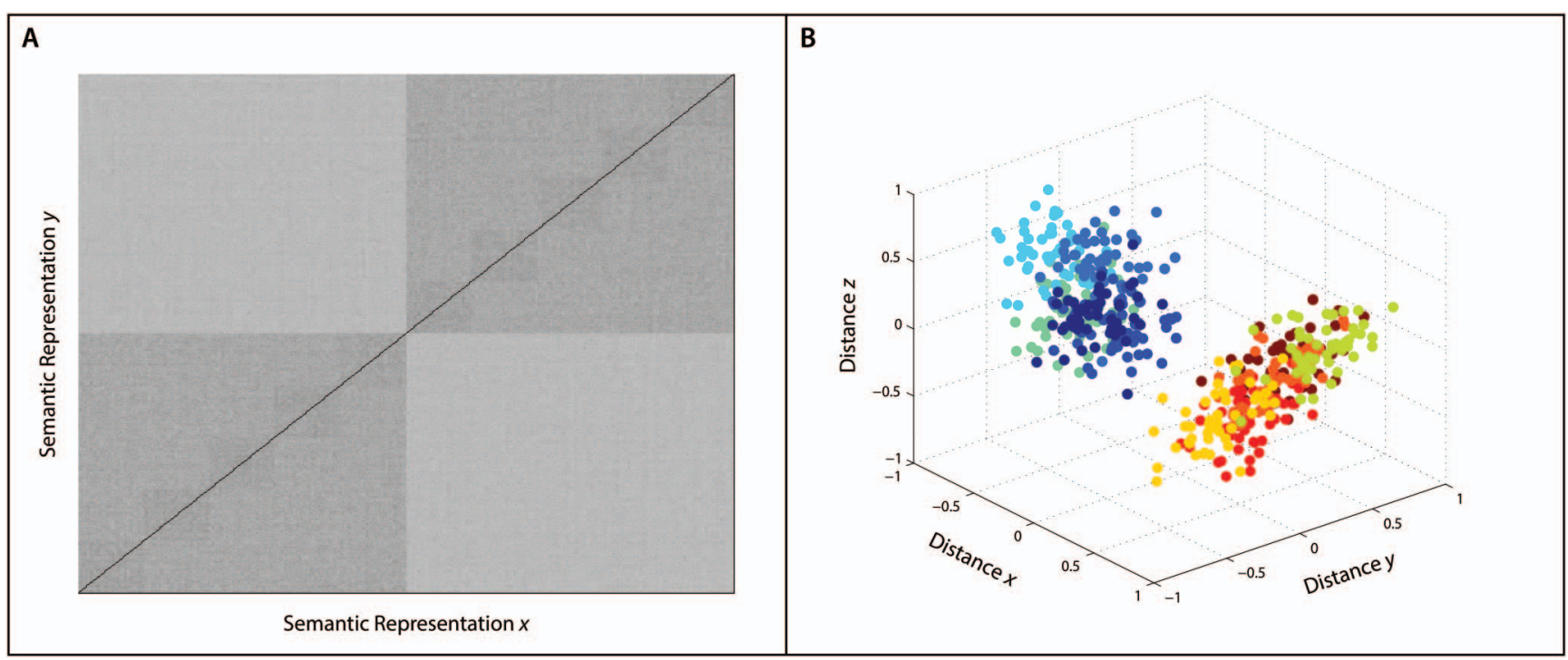

Figure 9. Figures A and B display the hierarchical structure embedded within semantic representations implemented in the reading model. (A) displays the mean cosine distance calculated between semantic representations for all possible pairings of the 500 items in each corpus, averaged over all corpora; darker shading indicates greater similarity between representations. (B) Plots the first three eigenvectors calculated as a product of principal coordinates analysis applied to the cosine distance matrix depicted in A; distinct colors (shading) are assigned to each semantic subcategory. See the online article for the color version of this figure.

mapping. Twenty-five homophonic homographs were included in the alphabetic shallow corpus reflecting the presence of 25 homophones in the phonology. Orthographic representations for each of the 50 nonwords in the phonology were constructed using corresponding phoneme to letter mappings.

Alphabetic deep. In the deep alphabetic system orthographic representations were constructed using the same procedure as outlined for the shallow alphabetic system with one variation, where $20 \%$ of mappings from orthography to phonology for each vowel were irregular. This was implemented by replacing within the alphabetic shallow representations on $20 \%$ of occasions for each vowel the letter assigned to the given vowel with one of the other four letters representing vowels. Pseudorandomization ensured that irregular mappings occurred an equal number of times for each vowel, with each alternative vowel used as the replacement an equal number of times, enabling the occurrence of letters representing each vowel to be controlled. Also, reflecting controls on consonants in the phonology each letter representing each consonant occurred an equal number of times within the corpus. Controls ensured that irregular mappings did not increase the number of homographs embedded within alphabetic deep corpora compared with alphabetic shallow systems. Note that introducing the deep alphabetic orthography in this way is an abstraction from natural orthographies, where irregular pronunciations (e.g., pint, compared with hint, tint, mint) overlap with pronunciation of other words containing similar letters (e.g., pine). In the current language this overlap was minimal. In total 25 homophonic homographs were embedded in each alphabetic deep corpus. Orthographic representations of nonwords were constructed using the regular phoneme to letter mappings defined for each alphabetic deep corpus.
Consonantal. Within a strictly consonantal system only consonants are represented (although in many consonantal scripts it is possible to add diacritics to indicate vowels). Within our implementation of the consonantal system the orthographic layer had four letter slots, organized as CCCC. An alphabet of 10 letters was constructed, each representing one of the 10 consonants in the phoneme inventory, and each letter was represented in terms of 12 features instead of 10 , in order that the input complexity of the consonantal system was similar to the other orthographic systems (but meaning that two of the 50 units in the orthographic input layer were always inactive for the consonantal orthographic system). As for the alphabetic simulations, the number of occurrences of each letter in each position was balanced. As vowels are not represented in this system the number of homographs is larger than the number of homophones, consonantal corpora contained on average 34 homophonic homographs $(M=33.6, S D=2.91)$. Orthographic representations were also created for the nonwords using the consonant to letter mappings as defined above. ${ }^{8}$

Alphasyllabic. Within alphasyllabic systems a basic grapheme indicates a consonant, with a diacritic added to indicate its combination with a particular vowel (in contrast to consonantal languages vowels must be indicated). In our implementation of the alphasyllabic system the orthographic layer consisted of four slots, organized $\mathrm{C}[\mathrm{CV}] \mathrm{CC}$, with $\mathrm{C}$ slots defined by 12 units and the $[\mathrm{CV}]$ slot defined by 14 units, to simulate a diacritic added to a conso-

\footnotetext{
${ }^{8}$ In some languages with consonantal orthographic systems words with similar meanings are expressed with consonantal roots where the vowels vary (e.g. Hebrew). However, this is not present in all languages with consonantal orthographic systems and, therefore, we do not represent that aspect of the language structure here.
} 
nantal character. Prototypes were produced for each consonant and used to create five unique 14 unit vectors for each consonant, each of which represented the consonant in combination with one of the five possible vowels. Prototypes ensured that 12 features were shared between representations of the same consonant with $p=.8$, while the remaining two features were shared with $p=.5$. This ensured that each representation of a given consonant was more similar to other representations of the same consonant and the signal indicating the vowel was distributed across the entire consonant-vowel slot. Because of the regularity of the mappings within this system the 25 homophones embedded in the orthography result in 25 homophonic homographs within each alphasyllabic corpus. Orthographic representations were also created for all 50 nonwords using the phoneme to grapheme mappings specific to each alphasyllabic corpus.

Syllabic. In syllabic systems a distinct grapheme encodes each syllable. No subcomponent of the orthography can be identified as denoting distinct subsyllabic segments. In our implementation of a syllabic system the orthographic layer consisted of single slot defined by 50 units. A unique 50 unit binary feature vector $(\mathrm{p}($ active $)=0.5)$ was created to form the orthographic representation of each syllable (CCVCC) within the corpus. There were 25 pairs of homophonic homographs in this orthographic system, reflecting the 25 homophone pairs in the phonology.

Logographic semantically transparent. In many logographic systems components of the orthography provide probabilistic information regarding the semantic category of a word. To explore the effects of this property we constructed a logographic system in which there was greater overlap of orthographic features between words within the same semantic subcategory than words within the same semantic higher level category yet not within the same subcategory, and greater overlap of orthographic features between words within the same higher level category than between words in different high level semantic categories. Thus, aspects of the orthography approximated the semantic structure of the items. Logographic semantically transparent orthographic representations consisted of a unique 50 unit binary feature vector $(\mathrm{p}$ (active) $=$ 0.5 ). The first 30 features were more likely to be shared with items within the same semantic category. These 30 features were split into two sets of 15 features, each subdivided in into five sets of three features, with each subset assigned a given semantic subcategory. The probability that a given orthographic feature was active was dependent on the words distance from the semantic category it was attributed to: $\mathrm{p}$ (active) $=0.8$, for a feature assigned to the same semantic subcategory as the given word; $\mathrm{p}$ (active) $=0.6$ for a feature assigned to the same high-level semantic category as the given word; and $\mathrm{p}$ (active) $=0.36$ for a feature assigned to a high-level semantic category that differed from that of the given word.

Logographic semantically opaque. To isolate the effects of semantic transparency from logographic structure we also constructed logographic semantically opaque systems. In the case of monosyllabic words semantically opaque logographic systems would differ from syllabic systems only in that two items with identical phonological representations that differ in their meanings will have distinct orthographic representations in the logographic language, but would be identical in phonology and orthography in the syllabic system. Within our implementation of logographic and syllabic systems the orthographic layer consisted of a single 50 unit slot. For logographic semantically opaque systems a unique 50 unit binary feature $(\mathrm{p}($ active $)=0.5$ ) vector encoded each word within the corpus, thus, there were no homographs in this orthographic system.

\section{Training}

The model was trained to map between phonological, semantic, and orthographic representations for all words in the training set. Each training trial ran for 12 time steps, with an integration constant of 0.33 , which meant that activation passed from one layer to another gradually and accumulatively, with a full pass of activation every three time steps.

The training regimes were identical across each orthographic system. There were two stages to the training. During preliteracy training the model was exposed to phonological and semantic representations of words, to simulate the child's exposure to spoken language before learning to read (Harm \& Seidenberg, 1999). Literacy training comprised learning to map orthographic forms of words onto phonological and semantic representations while also maintaining performance on the preliterate tasks. This was to simulate the interleaving of exposure to reading tasks and spoken language while children are learning to read.

Preliteracy training consisted of four tasks that varied in their probability of occurrence: phonological retention task, $p=.1$; semantic retention task, $p=.1$; speech comprehension, $p=.4$; and speech production, $p=.4$.

For phonological retention trials, the model was given the word's phonological representation, and was trained to stably maintain that representation over the 12 time steps. Words were randomly selected from the corpus. The phonological representation was clamped to the phonological layer for time steps $0-7$. The target (phonological representation of the selected word) was provided from time step 8-12. For this and the following tasks the presentation of the target was only made at the point when activation had passed fully from the layer provided with the input to the layer representing the required output (i.e., eight time steps).

Semantic retention trials required the model to sustain semantic representations in the semantic layer over time. A randomly selected semantic representation from the training corpus was clamped to the semantic layer for time steps $0-7$. The target was then provided from time steps $8-12$.

Speech production trials trained the network to map from semantics to phonology, to simulate spoken word production. A randomly selected word was taken from the corpus for each trial. Its semantic representation was clamped to the semantic layer for time steps $0-7$. For time steps $8-12$ the word's phonological representation was provided as target to the phonological layer.

Speech comprehension trials trained the network to map from phonology to semantics. A randomly selected word's phonological representation was clamped to the phonological layer for time steps $0-7$. For time steps $8-12$ the word's semantic representation was presented to the semantic layer as a target.

Note that the preliteracy training was identical for all simulations of the distinct orthographies, because the phonological and semantic representations were identical, just the orthographic forms varied. Networks were trained on a total of 150,000 preliteracy trials before the onset of literacy training. This ensured that networks were able to perform all tasks to a high degree of 
accuracy before the onset of literacy training: for the phonological retention and semantic retention tasks, accuracy was 100\%; for the speech comprehension task (phonology to semantics mappings), accuracy was $95 \%$ (this was the maximum possible because $5 \%$ of the training patterns were indistinguishable without additional implementation of contextual information for meaning, e.g., Chang et al., 2019, because of the inclusion of homophones in the training set); and for speech production (semantics to phonology mappings) accuracy was $100 \%$. In each case, an accurate response was when the output at the final, 12th step was closest to the target than to any other pattern in the training set, using cosine distance.

Literacy training consisted of the four pretraining tasks (phonological retention task, $p=.05$; semantic retention task, $p=.05$; speech comprehension, $p=.25$; speech production, $p=.25)$ and an additional reading task $(p=.4)$.

Reading trials trained the network to map from orthography to simultaneously produced phonological and semantic representations. For time steps $0-7$ the orthographic representation of a randomly selected word was clamped to the orthographic layer. During time steps $8-12$, the phonological representation of the word and the semantic representation of the word were presented to the phonological layer and semantic layer of the network, respectively, as a target and error back propagated. Networks were trained on a further 100,000 training trials with pretraining and reading trials randomly interleaved. At the end of literacy training all simulations performed pretraining tasks to the same level of accuracy as displayed before literacy training. Performance on reading tasks is detailed in later sections of this article (see Examining the Effects of Orthographic Transparency: Mono-Syllabic Simulations). Words were randomly selected from the training corpus with equal probability for use on training trials. Continuous recurrent backpropagation (Pearlmutter, 1989; Rumelhart, Hinton, $\&$ Williams, 1986) was used to train the model with a learning rate of 0.1 . Connection weights within the network were initiated with random weights in a uniform distribution in the range $(-1,1)$. Eight simulation runs, each with different randomizations of order of patterns selected and initial weight states, were trained for each orthographic system. All training and testing parameters were the same across orthographic systems.

\section{Examining the Effects of Orthographic Transparency: Mono-Syllabic Simulations}

The preliterate training environment was controlled across simulations of each orthographic system because phonological and semantic representations of words were identical across each orthography; thus, there was no variation according to orthographic system in the preliteracy training results. Furthermore, all simulations attained maximal levels of performance on all preliteracy training tasks before the onset of literacy training.

We first report performance on the model's acquisition of reading from the different orthographic systems. A key issue in computational modeling of alphabetic orthographies is the ability of the model to generalize to previously unseen items, such as in a nonword pronunciation task. Original criticisms of connectionist models of reading focused on poor generalization performance (e.g., Coltheart et al., 2001; Seidenberg \& McClelland, 1989), though subsequent connectionist models have effected this generalization (e.g., Harm \& Seidenberg, 1999; Plaut et al., 1996).
Nevertheless, nonword reading is a benchmark effect for any simulation of trained reading. Therefore, we also tested the model's ability to generalize pronunciation to previously unseen words (see online Supplemental Materials Section A: nonword reading). Then, we investigate the division of labor that emerges across the two pathways (orthography to phonology and orthography to semantics) in the model for reading pronunciation and comprehension. Finally, we provide an analysis of the differential effects of the orthographic systems on changes to the way in which the model represents structure in the phonological (and semantic, see online Supplemental Materials Section C) system. These analyses demonstrate the computational consequences of literacy on phonological and semantic processing across orthographic systems.

\section{Reading Acquisition}

For the reading acquisition tasks, we report performance on reading pronunciation, where the model's performance on phonological output given orthographic input is assessed. Then, we describe the model's performance on the reading comprehension task, where for given orthographic input, the model's semantic output is appraised. These results test the extent to which orthographic systems affect the trajectory of reading development, and the relative speed of learning to read for pronunciation and for comprehension.

Acquiring phonological decoding abilities under different orthographies. Each simulation was tested on its ability to accurately produce the phonological representation of a word when presented with its orthographic representation during training at 5,000 training patterns intervals. As for the measurement of accuracy for the speech production and speech comprehension tasks in the preliteracy model performance was recorded at the final 12th time step in the phonological layer of the network. The cosine distance between the output activation and all phonological representations in the training corpus was calculated. A word was recorded as accurately produced if its phonological representation was closest to the activation recorded in the phonological layer. Figure 10 displays the accuracy for all words in the set of eight simulation runs for each orthographic system during the first 100,000 literacy training trials. A clear distinction emerges from the onset of literacy training between subsyllabically transparent systems and the remaining systems in decoding acquisition rate with subsyllabically transparent systems rapidly reaching accuracy levels exceeding 70\% after 5,000 trials, whereas logographic and syllabic systems require approximately 40,000 trials to reach similar levels of performance.

Because of the greater number of homographs within the consonantal corpora some of the consonantal networks were only capable of achieving $92 \%$ accuracy in reading comprehension. A threshold of $90 \%$ accuracy was chosen to ensure comparisons across systems were conducted at a level of proficiency attainable for all networks. An alternative approach would have been to omit homographs from the analysis, but we wanted to ensure that we compared orthographies for the entire set of representations, including different effects of homography resulting from the orthographic system. A one-way analysis of variance (ANOVA) confirmed that networks differed in their reading for production acquisition rates, $F(6,49)=445.5, \eta^{2}=0.982, p<.001$. Six 


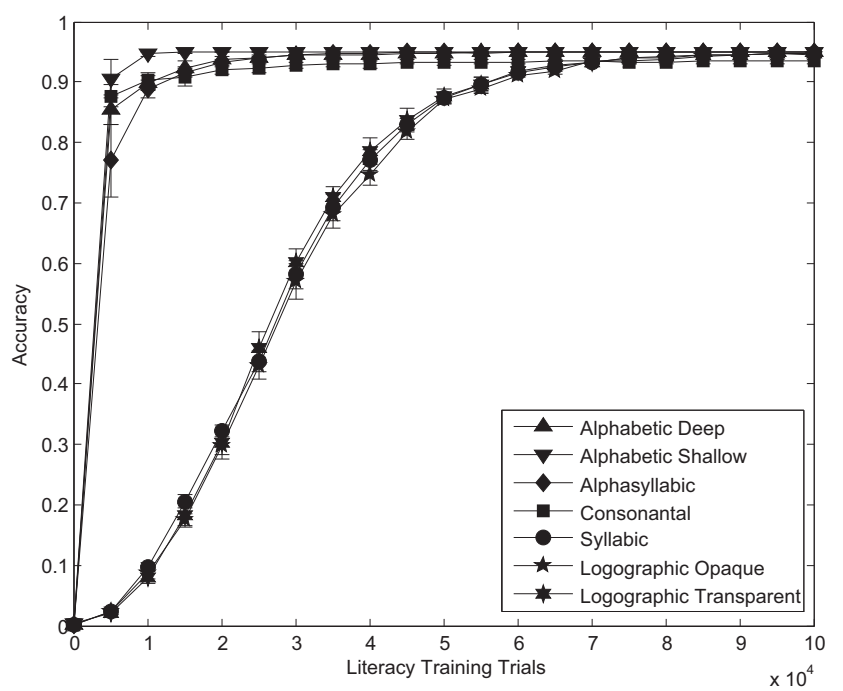

Figure 10. Phonological decoding accuracy during literacy training for each orthographic system.

two-sample $t$ tests were performed (Alphabetic Shallow, Alphabetic Deep; Alphabetic Deep, Alphasyllabic; Alphasyllabic, Consonantal; Consonantal, Syllabic; Syllabic, Logographic Transparent; Logographic Transparent, Logographic Opaque) to examine how individual orthographic systems differed from one another in their reading for production acquisition rates. This analysis revealed that shallow alphabetic networks reached 90\% accuracy before alphabetic deep networks $(M=-6,250, S D=3,952.8$, $t(14)=-3.162, p=.007)$. Alphabetic deep networks did not differ from alphasyllabic $(M=-2,500, S D=4,381.3$, $t(14)=-1.141, p=.272$ ), while alphasyllabic did not differ from consonantal networks $(M=3,125, S D=3,867.2, t(14)=1.616$, $p=.128)$. However, consonantal networks achieved $90 \%$ accuracy in orthographic to phonological mappings sooner than syllabic networks $(M=-46,250, S D=3,659.6, t(14)=-25.28$, $p<.001)$. There was no difference in acquisition rates displayed between syllabic and logographic transparent $(M=1,250, S D=$ $3,133.9, t(14)=0.797, p=.438)$. There was, however, a marginal difference between logographic transparent and logographic opaque networks $(M=-3,750, S D=2,500, t(14)=-3, p=$ $.010)$, although this was not significant when correcting for multiple comparisons.

The model replicated known findings that orthographic transparency increases the rate of phonological decoding acquisition. The increased acquisition rate of alphabetic shallow networks over alphabetic deep networks supports such conclusions from studies that control for exposure to training while witnessing reduced performance on word and nonword reading tasks in populations learning deeper alphabetic systems (Finnish vs. English: Goswami et al., 1998; Seymour et al., 2003; French vs. English: Bruck et al., 1997; Welsh vs. English: Hanley et al., 2004). Although comparing the number of trials required to achieve $90 \%$ accuracy on decoding tasks did not distinguish between alphasyllabic and alphabetic deep trials, there is a suggestion of a difference as indicated by Figure 10 that alphasyllabic networks were delayed in comparison with alphabetic deep networks at earlier stages of training. This aligns with the empirical data reported in Nag (2007) that describes delayed decoding acquisition in populations learning Kannada (alphasyllabic) in comparison with populations learning English (deep alphabetic). Also, as expected given the empirical data, logographic systems displayed the slowest decoding acquisition rates. In contrast, however, to data reported in Asfaha et al. (2009) that showed increased acquisition rates in populations learning Ge'ez scripts (alphasyllabic) compared with those learning Latin scripts (alphabetic), in our simulations syllabic systems displayed no decoding advantage over any other system. The model's failure to capture the observations reported in this study are likely because of the limitations of modeling only monosyllabic words and assumptions regarding the structure of phonological input to the system, we return to this issue in the General Discussion. Logographic transparent networks were marginally distinguishable from logographic opaque networks in their ability to learn orthographic to phonological mappings, although we are unaware of any existing empirical data examining the effects of semantic transparency on phonological decoding in logographic systems, the current model suggests that systematic relations between orthography and semantics may increase the rate of acquisition of phonological decoding abilities. We presume that this is because of systematic relationships between orthography and semantics increasing the rate at which orthographic to semantic mappings are learnt in logographic transparent networks; thus, decoding acquisition can benefit from early access to information from the orthographic to phonology via semantics. We will return to this issue in our examination of the division of labor within networks in section Division of Labor.

Acquiring reading comprehension abilities under different orthographies. The trajectory of learning orthography to semantic mappings was also examined (reading comprehension). To test accuracy on these mappings the model's output in the semantic layer was analyzed in the final time step (time step $=12$ ) for a reading trial and was compared with all semantic representations within the corpus. If activation in the semantic layer was closest to the target word then the item was recorded as read accurately. Figure 11 displays the accuracy of networks on this reading for comprehension task, as a proportion of items read within the corpus, over the course of literacy training. The model successfully learns to accurately map from orthographic to semantic representations for all words in the training corpus (allowing for variation in the number of homographs across systems) for all orthographic systems. In this respect it is comparable with previous models of reading and semantics (Harm \& Seidenberg, 2004; Monaghan, Shillcock, \& McDonald, 2004; Plaut et al., 1996).

Figure 11 shows, as was apparent in rates of phonological decoding acquisition, that a distinction can be made between reading comprehension acquisition rates displayed by systems that possess subsyllabic phonological structure in their orthography and those that do not, with subsyllabic phonological transparency leading to faster rates of acquisition in reading comprehension. The system with greatest phonological transparency (alphabetic shallow) appears to demonstrate the fastest rate of acquisition. As can be observed from Figure 11, phonologically transparent networks do not reach $100 \%$ accuracy in reading comprehension, this is because of the presence of homographs for which the semantic target cannot be determined from the orthography. 


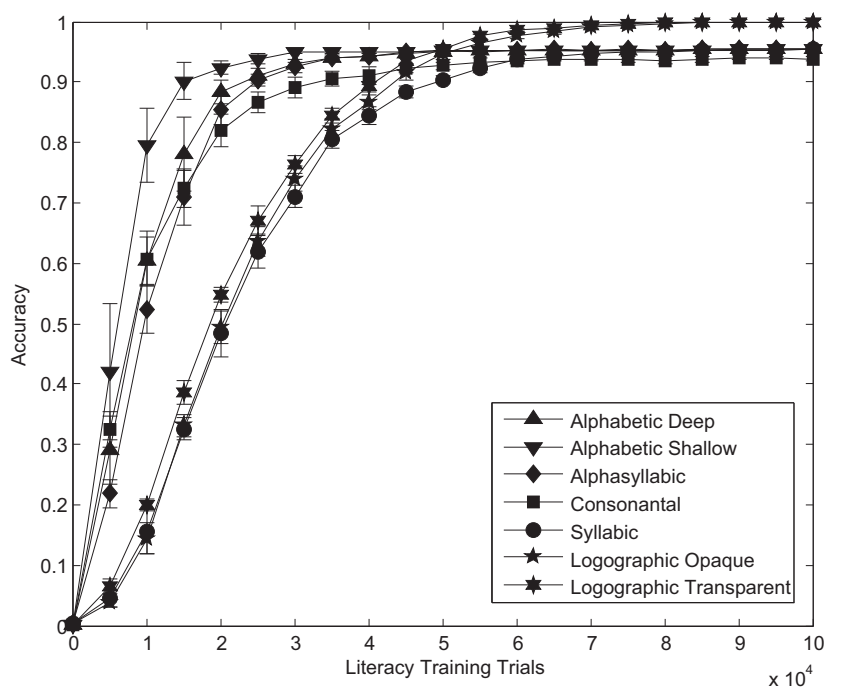

Figure 11. Reading comprehension accuracy across training.

To examine whether systems differed in the amount of training required to achieve $90 \%$ accuracy on the reading comprehension task a one-way ANOVA was performed. 90\% accuracy was selected as an appropriate threshold for comparison as it reflects a level of proficiency attainable for all networks. Orthographic systems differed significantly in this measure, $F(6,49)=78.19, \eta^{2}=$ $0.905, p<.001$.

To examine which individual orthographic systems differed in the training required to reach $90 \%$ accuracy on orthographic to semantic mappings we compared the number of training trials required to reach this level of proficiency using six two-sample $t$ tests (Alphabetic Shallow, Alphabetic Deep; Alphabetic Deep, Alphasyllabic; Alphasyllabic, Consonantal; Consonantal, Syllabic; Syllabic, Logographic Transparent; Logographic Transparent, Logographic Opaque). This analysis revealed that alphabetic shallow networks reached $90 \%$ accuracy before alphabetic deep networks $(M=-8,750, S D=3,720, t(14)=-4.704, p<.001)$. There was no difference in the number of trials required to reach this level of proficiency between networks trained on alphabetic deep and alphasyllabic systems $(M=-2,500, S D=3,953$, $t(14)=-1.265, p=.227)$. Consonantal systems achieved $90 \%$ accuracy before syllabic systems $(M=-16,250, S D=5,901$, $t(14)=-5.508, p<.001)$ as did logographic transparent systems $(M=7,500, S D=2,988, t(14)=5.020, p<.001)$. Differences between alphasyllabic networks and consonantal networks $(M=-8,125, S D=5,957, t(14)=-2.728, p=.016)$ and between logographic transparent networks and logographic opaque networks $(M=-2,500, S D=2,315, t(14)=-2.160, p=.049)$ were marginal, suggesting faster acquisition in alphasyllabic and logographic transparent systems, respectively, yet these differences were not significant after correcting for multiple comparisons.

For acquisition of reading comprehension abilities the level of semantic transparency embedded in the logographic transparent system had only a marginal effect on reading comprehension acquisition rate, this level of semantic transparency was also insufficient to override the advantage gained by the level of phonological transparency embedded in alphabetic, alphasyllabic and consonantal systems in learning orthographic to semantic mappings. Our modeling shows that within such an interactive system, phonological transparency significantly increased the rate of reading comprehension acquisition. This prediction does not fit with empirical data highlighted in Seidenberg (2011, 2013), such as increased comprehension abilities in English literates over Welsh literates (Hanley et al., 2004), which suggests a more complex relation between comprehension and phonological transparency. As raised by Seidenberg $(2011,2013)$ our modeling data support the position that should these more complex relations exist they are likely to be driven by factors beyond the monosyllabic word level, such as an effect of increased spoken language exposure, or result from systems not having full phonological and semantic knowledge of a language before literacy training (see, e.g., Monaghan and Ellis (2010) for connectionist simulations of reading where prior experience of words is related to partial vocabulary knowledge at the point of literacy training). We next tested this hypothesis.

Comparing learning trajectories for phonological decoding and reading comprehension. Our analysis shows that transparency modulates both phonological decoding and reading comprehension acquisition rates. As an initial step to examine and raise predictions for how transparency may affect the role of comprehension and decoding abilities in literacy acquisition we compared across systems and development the difference between the proportion of words networks were able to read for production and the proportion they were able to read for comprehension. A positive difference indicates a network is able to access the phonological form of a word from its orthography before it has learnt to access its semantic form, it is likely that the system makes use of the phonological information it is able to access from the orthography to support learning of orthographic semantic mappings. Conversely, should a network display a negative production-comprehension difference, this may suggest that the system recruits semantic information it is already able to extract from a given orthographic representation to support learning of orthographic to phonological mappings.

Figure 12 presents the difference between phonological decoding and reading comprehension accuracy for each orthographic system over the course of literacy training. As systems converge toward similar levels of accuracy on both tasks come the end of training, we examined whether there were differences between systems early on in training, in the first 250,000 training trials. A one-way ANOVA compared the difference between phonological decoding accuracy and reading comprehension accuracy summed over the initial 250,000 training trials. This revealed that orthographic systems differed significantly in this measure, $F(6,49)=$ $250.41, \eta^{2}=0.968, p<.001$. To examine how individual systems differed from one another systems were compared on this measure using six two-sample $t$ tests (Alphabetic Shallow, Alphabetic Deep; Alphabetic Deep, Alphasyllabic; Alphasyllabic, Consonantal; Consonantal, Syllabic; Syllabic, Logographic Transparent; Logographic Transparent, Logographic Opaque). Alphabetic shallow networks displayed greater accuracy in decoding relative to comprehension than alphabetic deep networks at this early stage of training $(M=0.359, S D=0.221, t(14)=3.246, p=.006)$. Alphasyllabic systems also displayed greater accuracy in decoding than comprehension relative to alphabetic deep networks $(M=$ 


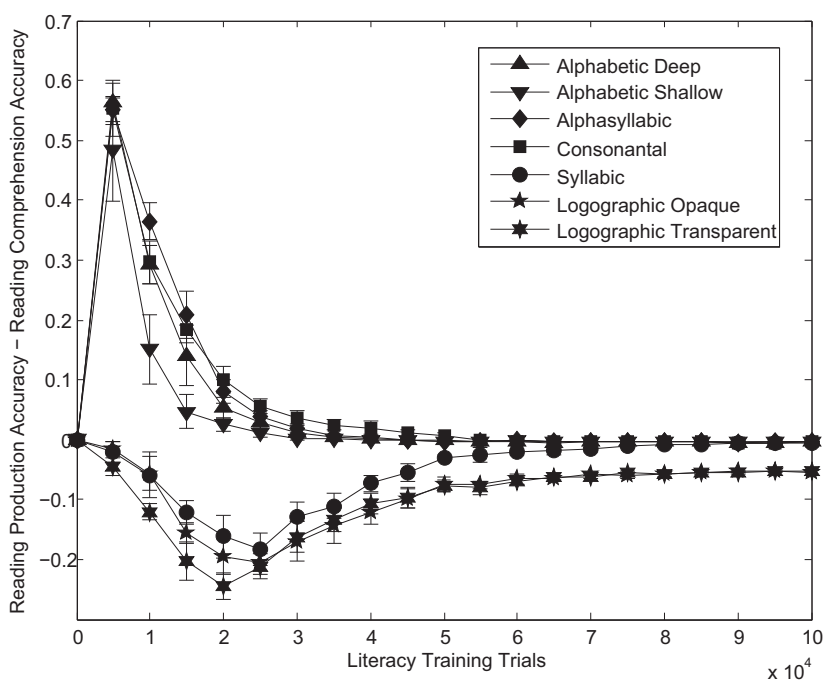

Figure 12. Difference between phonological decoding accuracy and reading comprehension accuracy across training.

$-0.520, S D=0.217, t(14)=-4.786, p<.001)$, although there was no difference between alphasyllabic and consonantal networks in this measure $(M=0.051, S D=0.154, t(14)=0.659, p=.520)$. Consonantal networks displayed substantially greater accuracy in decoding relative to comprehension than syllabic networks $(M=$ $1.737, S D=0.163, t(14)=21.28, p<.001)$. Whereas logographic transparent networks displayed greater comprehension accuracy relative to production accuracy compared with syllabic $(M=0.277, S D=0.144, t(14)=3.838, p=.002)$ and logographic opaque networks $(M=-0.194, S D=0.098$, $t(14)=-3.960, p=.001)$.

This analysis reveals that logographic and syllabic systems display better performance on orthographic to semantic mappings during the initial stages of literacy training, this bias is greatest in the logographic networks, with the inclusion of semantic transparency in the orthography increasing the comprehension bias further at early stages of training.

By contrast, systems that encode subsyllabic phonological structure display a strong decoding advantage at early stages of literacy training. All such systems are able at early stages of literacy training to decode the written form of a word yet not comprehend it for a large proportion of the training corpus. This decoding advantage reduces rapidly such that all subsyllabic phonologically transparent systems are able to both decode and comprehend written words with $90 \%$ accuracy before logographic and syllabic systems. The slot based structure of orthographic and phonological layers makes it easier for the model to identify grapheme to phoneme level correspondence. This implementation ignores the additional complexity faced by readers of alphabetic languages that must not only identify the letter sound mappings, but also learn how to blend sounds together to produce a given word's correct pronunciation (Hudson, Torgesen, Lane, \& Turner, 2012). The comprehension advantage in the transparent systems is likely because of the earlier generation of accurate phonological representations during training from orthographic input, which can then be used to support semantic representations using resources available in the direct orthography to phonology and the indirect orthography-phonology-semantics mappings. The extent to which activated phonological information via orthography is recruited to assist learning of orthographic to semantic mappings is explored in further detail in section Division of Labor. However, the model makes a clear prediction that in transparent orthographies decoding abilities should precede comprehension and that the increased ease with which such mappings are learnt should aid reading comprehension acquisition. As raised by Seidenberg $(2011,2013)$ there are few studies that directly compare phonological decoding and reading comprehension abilities and, therefore, whether transparency necessarily leads to increased rates of reading comprehension is still to be examined.

In contrast to the above pattern of development, logographic and syllabic systems display an early comprehension advantage, this advantage develops and recedes more gradually than the decoding advantage displayed by alphabetic, alphasyllabic, and consonantal systems. Our analysis shows that semantic transparency increases the comprehension bias with logographic transparent networks displaying a greater comprehension advantage in comparison to logographic opaque networks at earlier stages of training. The fact that syllabic and both logographic systems display a comprehension bias indicates that without systematic componential relations between orthography and phonology, orthographic to phonological mappings are more difficult for networks to learn than orthographic to semantic mappings given the architecture of the implemented reading network. Within this study the hidden layer connecting orthographic and semantic layers contains twice the resources of the hidden layer connecting orthographic and phonological layers (an assumption implemented in Harm \& Seidenberg, 2004). Therefore, given equal complexity of mappings, orthographic to semantic mappings should be learnt quicker in this system. This highlights further complexities of deducing the mechanisms driving observed behavior. Previous studies have argued that a greater spelling to meaning bias in Chinese compared with English is driven by the sublexical semantic structure embedded in Chinese orthographic representations (e.g., Yang et al., 2006). Yang et al. (2006) observed such a bias when training an implementation of the triangle model similar to that used in this article trained on 103 phonological, semantic, and orthographic patterns derived from Mandarin Chinese. Within our study using abstract logographic representations we replicate this bias, however, as in their study such a bias may also result from differences in the resources available for learning spelling to sound or spelling to meaning mappings within the system, or inherent differences in the complexity of these mappings irrespective of the additional sublexical semantic systematicity.

Conclusions: Reading acquisition. The simulations demonstrate a graded effect of both phonological and semantic transparency on both the acquisition of reading for production and reading for comprehension abilities. Orthographies that encode componential subsyllabic structure quickly learned orthography to phonology mappings while learning of orthographic to semantic mappings was delayed in such networks. However, learning orthographic to semantic mappings was still faster than systems that were not subsyllabically componential. This suggests that such networks may utilize phonological information they are able to extract from the orthography to aid mapping between orthography and semantics at least in early stages of training. Our data also suggest that semantic transparency may aid acquisition of reading comprehension and reading for production. In contrast to subsyllabically componential systems logographic and syllabic displayed greater rates of reading comprehension acquisition than reading for production, this comprehension bias be- 
ing greatest in logographic semantically transparent networks. This suggests that acquisition of decoding abilities in logographic and syllabic networks may conversely be assisted by activation of semantic information via the orthography, particularly if there are systematic relationships between orthography and semantics that aid learning of such mappings.

\section{Division of Labor}

In Reading Acquisition we have already seen that orthographic transparency leads to differences in reading comprehension and phonological decoding acquisition rates that suggest that transparency is likely to influence the division of labor across processing paths. Understanding the meaning of a written word can be solved either by mapping from orthography directly to semantics or via its phonological representation. Within this section we examine how the structure of the orthographic systems affects how the model solves the task of learning to read using the two routes available for each of the tasks. As previously mentioned, the model can learn to map from orthography to phonology via the direct route (O-P), or indirectly via orthography to semantics, and then semantics to phonology (O-S-P). Similarly, the model can learn the orthography to semantics mappings directly $(\mathrm{O}-\mathrm{S})$, or via the indirect orthography to phonology to semantics pathways (O-P-S). Note that the phonology to semantics and semantics to phonology pathways are already operating at a high degree of accuracy before the model is trained to learn mappings from orthography, so depending on the ease of learning the mappings from orthography to phonology and semantics can determine the extent to which the indirect pathways contribute to learning, and the degree to which they contribute changes as the reading system matures. Our analysis was interested in how each network successfully solves the reading task; therefore, the following analyses includes data only for words that at a given stage of training the network was able to read accurately. ${ }^{9}$

In this section we directly measured the activation passing along the different pathways in the model to determine the extent to which the indirect pathways were contributing to division of labor differently for each orthographic system, both through the course of learning to read, and also in the mature reading system, during the course of a single reading trial. Harm and Seidenberg (2004) examined the distribution of labor within a model of English reading using a similar approach. They observed that activation entering the semantic layer via the direct path $(\mathrm{O}-\mathrm{S})$ increased most rapidly, whereas activation entering the layer via the phonological path increased more slowly as phonological units needed to be activated first by the orthography before they could begin to exert an influence via the indirect path (O-P-S).

To examine how orthographic structure affects the flow of activation throughout the model during reading comprehension and phonological decoding, we recorded activity passing into the semantic layer and phonological layer via either the direct orthographic pathway or the indirect pathway during reading. An orthographic representation was clamped to the orthographic layer while the rest of the network was left to cycle freely for 11 further time steps. The log ratio between activation entering the semantic and phonological layer via the indirect path and activation entering via the direct path was calculated at each time step ( $\log [$ indirect path/direct path]). A log ratio of zero indicates the level of activation entering the layer via each path is equal, a positive value indicates increased activation via the indirect path, while a negative value indicates increased activation via the direct path. Simulations were tested on all words in the corpus and at each stage of literacy training.

Distribution of activation during reading production. Figure 13 displays the log ratio of activation entering the phonological layer via the indirect path (orthography to phonology via semantics, O-S-P) compared with activation entering the phonological layer via the direct path (orthography to phonology, O-P) calculated at time step 12 of reading trials at every 50,000 training patterns for each orthographic system averaged over all items and simulations. All systems display an initial negative ratio indicating that at the onset of literacy training there are greater levels of activation entering the phonological layer via the O-P path than the O-S-P path.

To understand the dynamics of activation entering the phonological layer over the course of reading trial in mature networks the $\log$ ratio between activation entering the layer via the O-S-P path and activation entering the phonological layer via the O-P path was also calculated at each time step of a reading trial once networks were able to perform both reading comprehension and phonological decoding tasks for $90 \%$ of words in the training corpus (a 90\% threshold ensures all systems are able to achieve this level of performance and maturity is comparable across systems). This measure is plotted in Figure 14 showing the average ratio for each system at each time step averaged across all items and simulations.

To test whether differences in orthographic structure generate differences in the ratio of activation entering the phonological layer in mature systems (90\% accuracy on phonological decoding and reading comprehension measures) the mean ratio across all items was summed across all time steps for each simulation. Systems were then compared by simulation on this measure using a one-way ANOVA. This revealed a difference between systems in the ratio of activation entering the phonological layer via O-S-P and O-P paths, $F(6,49)=4.11, \eta^{2}=0.335, p=.002$. Six two-sample $t$ tests were performed to examine how individual systems differed from one another (Alphabetic Shallow, Alphabetic Deep; Alphabetic Deep, Alphasyllabic; Alphasyllabic, Consonantal; Consonantal, Syllabic; Syllabic, Logographic Transparent; Logographic Transparent, Logographic Opaque), these tests showed that less activation entered the phonological layer via the O-S-P path in alphasyllabic systems compared with consonantal systems $(M=-5.516, S D=4.005, t(14)=-2.754, p=.016)$, although this was no longer significant with Bonferroni correction. No other comparison proved significant $(|\mathrm{t}|<1, p>.35$ ).

Although the analysis conducted on mature networks reveals moderate differences between systems in their use of O-P and O-S-P paths during phonological decoding, data presented in Figure 13 suggests that systems encoding subsyllabic structure display a substantially different developmental trajectory to those systems that only encode structure at the syllabic level and beyond. There is a suggestion of a difference as indicated by the Figure at earlier stages of training with greater O-P route bias in subsyllabic transparent systems whereas at later stages of training this trend is reversed, with a suggestion of

\footnotetext{
${ }^{9}$ In the online Supplemental Materials (Section B), we also report the model's activation of the phonological and semantic representations of each word over the 12 time steps of a reading trial, to demonstrate how the model gradually accumulates information about the word. Direct pathways provide rapid information, whereas slower activations indicate a greater role for activation contributed by the indirect pathways.
} 


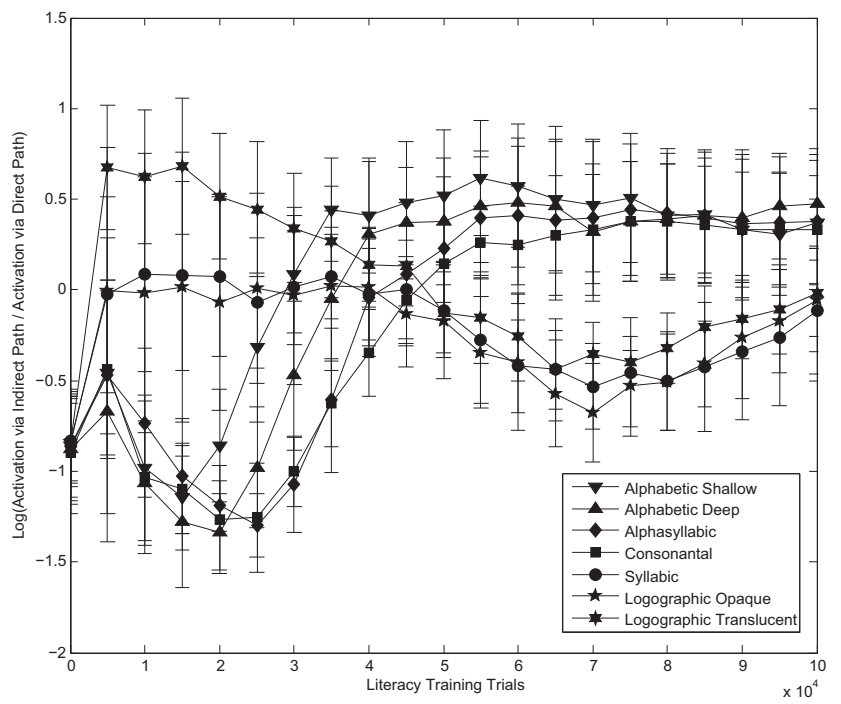

Figure 13. Log ratio of activation entering phonological layer via indirect path/via indirect path during reading trials across training averaged over all items and simulations.

greater O-S-P bias in systems that encode phonological information at subsyllabic levels. This likely reflects the fact that subsyllabic transparent networks are able to rapidly learn orthographic to phonological mappings relative to orthographic semantic mappings and, therefore, rely heavily on the direct orthography to phonology route at early stages on training, then as orthographic to semantic mappings become more effective information via the indirect orthographic to phonology via semantics route becomes increasingly influential such that by the end of training activation entering the phonological layer from both routes is approximately equal. By contrast syllabic and logographic networks do not learn orthographic to phonological mappings as rapidly; therefore, at early stages of training activation entering the phonological layer is approximately equal from both O-S-P and O-P paths, as accuracy on orthographic to phonological mappings in-

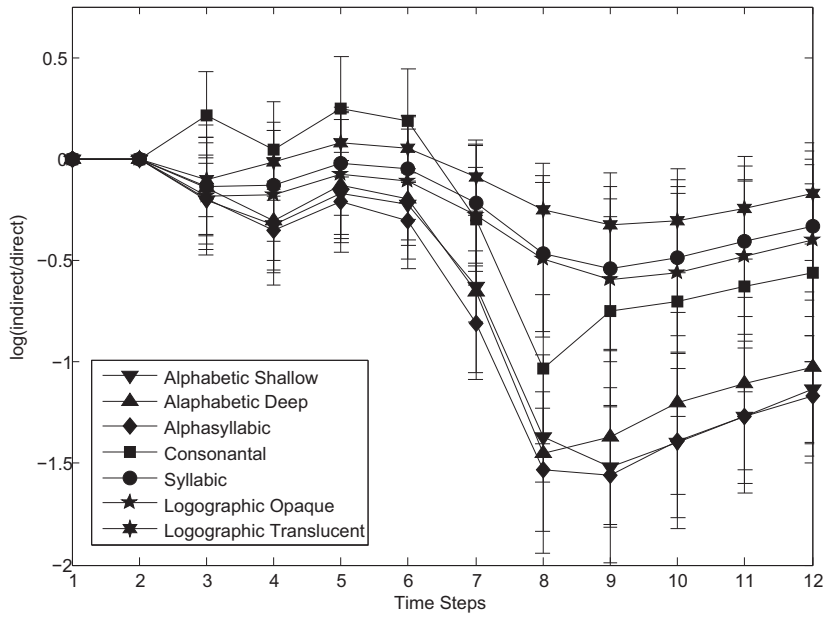

Figure 14. Log ratio of activation entering phonological layer via indirect path/via direct path over the course of a reading trial in trained $(90 \%$ reading comprehension and phonological decoding accuracy) networks. creases the magnitude of activation via the direct orthography to phonological path increases, although by the end of training there appears a trend toward an equivalent influence from both O-P and O-S-P paths.

Distribution of activation during reading comprehension. To examine how orthographic structure affects the flow of activation throughout the model during reading comprehension, we performed the same analysis on measures of activation entering the semantic layer via direct (orthography to semantics, O-S) and indirect paths (orthography to semantics via phonology, O-P-S).

The log ratio between activation entering the semantic layer via the indirect phonological path and activation entering the semantic layer via the direct path was calculated at time step 12 of reading trials for all items and simulations every 50,000 training patterns. Figure 15 shows how this ratio averaged over all items and simulations changed over the course of literacy training for each orthographic system. The dynamics of activation entering the semantic layer via O-S and O-P-S paths were also examined over the course of a single reading trial in networks once they were able to perform reading comprehension and phonological decoding tasks for $90 \%$ of words in the training corpus (90\% threshold chosen to ensure comparisons across systems could be conducted at equivalent levels of performance), the results of which are shown in Figure 16.

To examine whether the structure of the orthographic system affected these dynamics a one-way ANOVA compared orthographic systems by simulation the log ratio of activation entering the semantic layer via the O-P-S path over the O-S path summed across the entire reading trial (time steps $1-12$ ) in mature networks. This revealed an effect of orthographic structure, $F(6$, 49) $=76.51, \eta^{2}=0.904, p<.001$. Six two-sample $t$ tests were also performed to examine how individual systems differed from one another (Alphabetic Shallow, Alphabetic Deep; Alphabetic Deep, Alphasyllabic; Alphasyllabic, Consonantal; Consonantal,

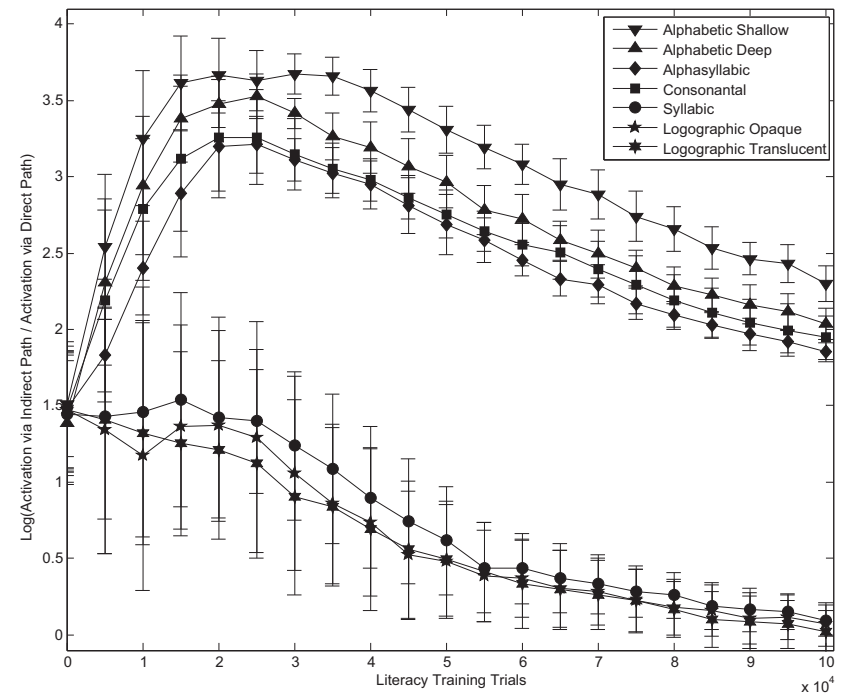

Figure 15. Log ratio of activation entering semantic layer via indirect path/via direct path during reading trials across training averaged over all items and simulations. $\mathrm{AS}=$ alphabetic shallow; $\mathrm{AD}=$ alphabetic deep; $\mathrm{AlSl}=$ alphasyllabic; Con $=$ consonantal; Syll $=$ syllabic; $\mathrm{LO}=\operatorname{logo}-$ graphic semantically opaque; LT = logographic semantically transparent. 


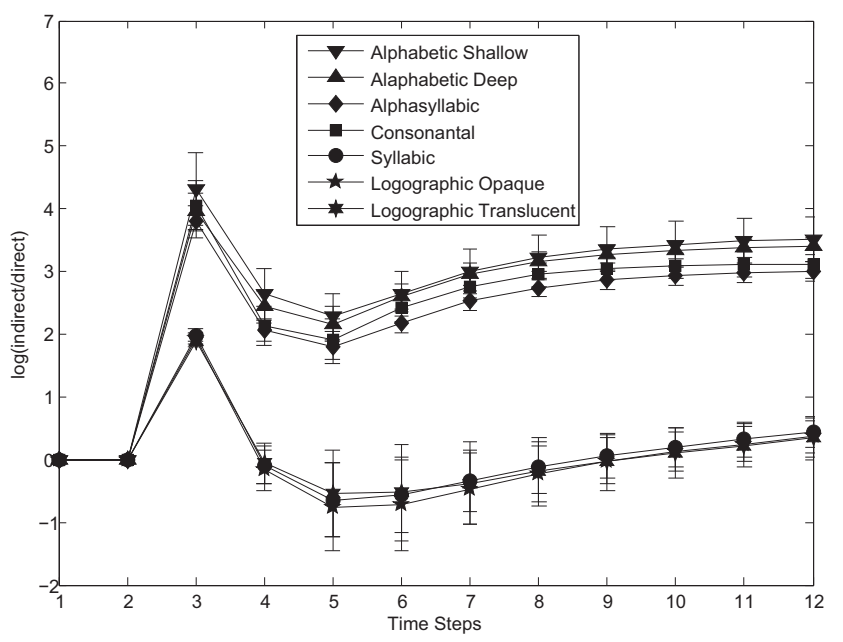

Figure 16. Log ratio of activation entering semantic layer via indirect path/via direct path over the course of a reading trial in trained $(90 \%$ reading comprehension and phonological decoding accuracy) networks.

Syllabic; Syllabic, Logographic Transparent; Logographic Transparent, Logographic Opaque). These tests suggest that alphabetic deep networks displayed a greater bias toward activation entering the semantic layer via the O-P-S phonological path than alphasyllabic networks $(M=3.765, S D=2.535, t(14)=2.970, p=.010)$, although this difference was not significant after correcting for multiple comparisons. Consonantal networks displayed a far greater bias toward activation entering the semantic layer via the O-P-S path compared with the O-S path when compared with ratios displayed by syllabic networks $(M=27.371, S D=4.576$, $t(14)=11.963, p<.001)$. Remaining comparisons did not indicate a difference between systems ( $|\mathrm{t}|<1.2, p>.25)$.

Data presented in Figure 15 suggest that all orthographic systems displayed increased activation flowing into the semantic layer via the O-P-S path at early stages of training; yet, this bias decreased over the course of literacy training. The bias displayed by all networks before literacy training of increased activation entering the semantic layer via the O-P-S path is likely to reflect the preestablished learning of phonological to semantic mappings. Given that this bias reduces from the onset of literacy training in logographic and syllabic simulations, this indicates a growing influence of activation from the O-S path. Logographic and syllabic systems displayed approximately equal activation flowing into the semantic layer from each route by the end of literacy training. The bias toward greater activation via the O-P-S path is much greater in alphabetic, alphasyllabic, and consonantal networks at all stages of development. This difference between networks emerges rapidly once literacy training commences and is greatest at earlier stages of training. In direct contrast to the pattern of development displayed by logographic and syllabic systems, alphabetic, alphasyllabic, and consonantal networks display an immediate and rapid increase in O-P-S bias from the onset of literacy training. This bias peaks after approximately 30,000 literacy training trials, before decreasing for the remainder of training. This pattern indicates an increasing influence of the O-P-S path at early stages of literacy training, with the O-S path becoming more influential as training increases after this point. By the end of training alphabetic, alphasyllabic, and consonantal networks continue to display a far greater
O-P-S bias compared with logographic and syllabic networks. This contrasts with some arguments in the literature that suggest the O-P-S path is likely to become largely redundant in proficient readers (see Levy et al., 2008, 2009) However, it is not possible to rule out this result arising should the model be exposed to extensive literacy training with pressure to activate semantic knowledge rapidly, therefore, favoring O-S path activation.

Figure 16 shows that the ratio of activation entering the semantic layer via the indirect path and direct path varies over the course of a single reading comprehension trial. All orthographic systems displayed an initial increase in activation into the semantic layer via the O-P-S path early in the reading trial. In logographic and syllabic simulations this increased activation via the O-P-S path declined rapidly and remained low for the remainder of the trial. In contrast alphabetic, alphasyllabic, and consonantal simulations displayed a greater initial increase in activation via the O-P-S path. This also declined rapidly, however, in contrast to syllabic and logographic simulations, the O-P-S path contribution remained greater in such networks for the remainder of the trial and increased steadily from time step 3 onward.

All networks displayed an initial spike in activation from the phonological path at time step 3, although this spike is far larger in subsyllabic transparent networks. This coincides with activity in the semantic layer becoming more distant from the target (see online Supplemental Materials Section B), suggesting that this initial spike may reflect noise coming from the phonological layer before it begins to settle on the word's phonological form. Following the initial spike in activation from the O-P-S path, logographic and syllabic networks displayed an approximately equivalent level of activation entering the semantic layer from both O-S and O-P-S paths. Although mean ratios indicate a small bias toward O-S path activation at earlier stages of the trial, activation from the O-P-S path steadily increases over the course of the trial such that activation through both paths are equivalent by the end of the reading trial. Subsyllabic transparent systems display a similar trough in O-P-S bias at time step 5 with the O-P-S bias increasing for the remainder of the reading trial. Richardson et al. (2011) observed activation of the O-P-S path at both early and late stages of word reading, and it is possible that this is captured in our model by the two peaks we observe in O-P-S path activation. Richardson et al. (2011) interpreted this activation as potentially reflecting mapping at multiple grain sizes; however, given that we observe similar peaks in activation for both transparent and nontransparent systems, so for some systems finer grain-size than the whole word would not assist the mapping between orthography and phonology, this explanation would not account for this pattern in our computational model. Instead, the initial peak is because of initial noise coming from the phonological layer before it settling on the phonological representation of the target, the later peak emerges as a consequence of increasing activation from the phonological layer as an increased number of phonological features of the target word are activated.

Discussion: Distribution of activation during reading. These results describe how the activation of different routes within the reading system may be affected by orthographic transparency both over the course of development and in mature systems. Analysis of ratios capturing differences in activation of indirect and direct paths in mature networks during phonological decoding shows that alphabetic and alphasyllabic systems display greater levels of activation passing through the direct orthography to phonology path than the indirect 
semantic path in comparison to syllabic and logographic systems. Though, as indicated by Figure 13, this difference may alter if networks are exposed to further literacy training. By contrast a more stable difference over development in use of direct and indirect paths is displayed during reading comprehension between systems that encode subsyllabic structure in their orthography and those that do not. Mature logographic and syllabic systems display greater activation via $\mathrm{O}-\mathrm{S}$ paths than O-P-S paths during reading comprehension in comparison to systems that encode phonological information at subsyllabic levels. Further, as suggested by Figure 15, this difference emerges early in development and remains present over the course of development. In contrast to our predictions about the model's performance, there was no significant influence of semantic transparency on the distribution of activation across direct and indirect paths during reading comprehension or phonological decoding.

Together these data argue for a graded effect of phonological transparency on the distribution of activation across indirect and direct paths during both phonological decoding and reading comprehension. This aligns with cognitive neuroscientific studies comparing activation through ventral (direct) and dorsal (indirect) paths across populations that differ in the orthographic transparency of the system on which they were trained. As captured by the model, Paulesu et al. (2000) observed a dorsal bias in activation in a shallow orthographic system (Italian) compared with a deeper orthographic system (English). Further, Kiyosawa et al. (1995) observed a dorsal bias for individuals when they read a transparent orthography (Kana) compared with a nontransparent orthography (Kanji; see also Thuy et al., 2004). More recent data reported in Rueckl et al. (2015) is also largely consistent with the results of our simulations, although the measures recorded in their study limit direct comparisons as they examined the level of overlap between networks activated when processing speech compared with networks activated when processing written words whereas within our model connectivity between phonological, semantic and orthographic processing components is controlled across orthographic systems. However, they observed stronger coupling between speech and text processing networks in regions generally associated with phonological processing for the most transparent system under investigation (Spanish) relative to two less transparent systems (English and Hebrew), whereas such deeper orthographic systems generated greater coupling in regions more closely associated with semantic processing. In our simulations networks processing transparency increased activity entering the phonological layer via direct connections between orthography and phonology than activation entering via indirect routes from semantic regions, while the converse is true for information entering the semantic layer, with less transparent systems displaying greater activity entering via direct orthographic to semantic connections. It is possible such properties of the system are replicating the results observed in Rueckl et al. (2015) should this greater activity displayed by the model reflect increased likelihood of a region being observed as active both when processing spoken and written words. However, in contrast to the predictions of the triangle model implemented in this study comparisons between opaque (Chinese) and transparent (Hebrew, English, and Spanish) systems in Rueckl et al. (2015) reveal only minor differences in activation profiles.

As the model used in this study was a learning model it was also possible to examine how the division of labor in networks developed over the course of literacy training. Previous neuroimaging studies (Pugh et al., 2000; Shaywitz et al., 2002) and computational modeling studies (Harm \& Seidenberg, 2004) have demonstrated increased activation of the indirect path during reading comprehension in early stages of literacy training on a deep alphabetic system such as English. Our modeling replicates this finding and indicates that phonological transparency leads to distinct patterns of development. By contrast, semantic transparency (as implemented in this study) appears to have little impact on the development of the distribution of labor.

The division of labor is not predefined but instead develops as the statistical learning algorithm applied attempts to find the most efficient means of mapping between orthography and semantics given the constraints imposed by the architecture and learning environment. As orthographic structure is the only factor to differ between simulations we can be confident that differences in the division of labor observed between simulations are driven by this variable and not semantic or phonological structure, factors that were not controlled in previous modeling studies (Yang et al., 2013). The above data provides an explicit description of how differences in processing observed in neuroimaging studies may emerge as a consequence of the same underlying architecture and learning mechanisms configuring around orthographic systems that differ in their semantic and phonological transparency.

\section{Literacy Effects on Phonological Processing}

As discussed in section Effects of literacy on representational structure there are existing behavioral (e.g., De Gelder \& Vroomen, 1992), neural (e.g., Brennan et al., 2013), and computational (e.g., Smith, Monaghan, \& Huettig, 2014b) data supported by theoretical models (e.g., Ziegler \& Goswami, 2005) that argues that phonological processing is affected differentially by orthographic transparency, with transparency leading to finer-grain processing. In this section we, therefore, examine whether differences in the structure of phonological representations can be observed across orthographic systems. This is possible as phonological representations are controlled across orthographic systems and, therefore, differences that emerge will result entirely from constraints imposed by a given orthography on mappings from orthography to phonology. ${ }^{10}$

In the following sections, we first examined effects on the structure of representations activated during trials in which orthographic representations are also directly activated. We then move on to examining effects on representations during phonological retention trials in which orthographic representations are not active.

\footnotetext{
${ }^{10}$ Given that orthographic effects have been observed on phonological processing, which it has been argued (see section Effects of literacy on representational structure) are driven by the extent to which systematic relations are embedded in the orthography to phonological representations, it is possible that analogous effects of literacy on semantic processing could be observed for orthographic systems in which systematic relations exist between orthography and semantics, such as are represented in the semantic radicals of traditional Chinese characters. Although, this may be less likely in the semantic domain than phonological domain given semantics is multidimensional and multimodal (a property absent from the current implementation). We tested this by examining whether the structure of semantic processing displayed by the model post literacy training is influenced by semantic transparency either when orthographic information is activated explicitly (orthographic representation activated in the orthographic layer) or in the absence of explicit activation of orthographic representations (no orthographic activation in the orthographic layer). To our knowledge, there are as yet no such studies that have investigated this issue. These analyses can be found in the online Supplemental Materials (Section C).
} 
Measures were recorded only for words that the networks were able to read accurately in terms of activating the correct phonology and semantic representations when presented with their corresponding orthographic form; thus, ensuring that our results only reflect changes to the representation of words for which the network possesses functional knowledge.

To ensure systems were comparable at equivalent levels of reading proficiency networks were tested once they were able to map from orthography to phonology and semantics accurately for $90 \%$ of words in the training corpus. A threshold of $90 \%$ was chosen as this was a level of performance attainable for all systems.

To examine whether the orthographic system on which the model received training altered the componential nature of phonological processing we determined the extent to which similarities between words were best reflected at the phoneme level (thus, reflecting phoneme-level segmentation) or whether similarities were just in terms of global similarity across the whole word, regardless of phoneme-level similarities. This was done by testing the model on a set of 400 word triplets that were controlled to be similar in terms of the number of phonological features that they shared over the whole word, but differed in terms of the number of phonemes that they shared. Each of the 400 sets was composed of a control word (Control word), a word that shared overlapping phonemes with the control word (Phoneme overlapping word), and a word (Feature overlapping word) that shared an equal number of phonological features with the control word as the phoneme overlapping word $(M=35.25, S D=2.39)$ but fewer overlapping phonemes than the phoneme overlapping word (Number of phonemes shared between: Phoneme overlapping words and Control words: $M=2.04, S D=0.20$; Feature overlapping words and Control words: $M=0.82, S D=0.45$ ).

Simulations were tested on their processing of these 400 sets of words during both reading and phonological retention tasks. Activation in phonological layers was recorded while processing each word on each task. For each word pair (i.e., Control word, Feature overlapping word; Control word Phoneme overlapping word) the cosine distance (1-cosine angle between vectors) between activation in the phonological layer was calculated. The following results report the ratio (Pdist/Fdist) of these two distances (i.e., Distance between Control word and Phoneme overlapping word/Distance between Control word and Feature overlapping word). Lower Pdist/Fdist ratios indicate that phonological representations with shared phonemes are more similar than those that only share the same number of phonological features, indicating phoneme-level processing.

Granularity of phonological processing during reading. Figure 17 displays the Pdist/Fdist ratio based on activation in the phonological layer at time step 12 of reading tasks displayed by networks able to accurately map from orthographic representations to phonological and semantic representations for $90 \%$ of words within the training corpus. A one-way ANOVA conducted on this measure showed that systems differed in Pdist/Fdist ratios at time step 12 of reading tasks, $F(6,49)=16.92, \eta^{2}=0.674, p<.001$. Six two-sample $t$ tests examined differences between individual orthographic systems (Alphabetic Shallow, Alphabetic Deep; Alphabetic Deep, Alphasyllabic; Alphasyllabic, Consonantal; Consonantal, Syllabic; Syllabic, Logographic Transparent; Logographic Transparent, Logographic Opaque). This analysis revealed

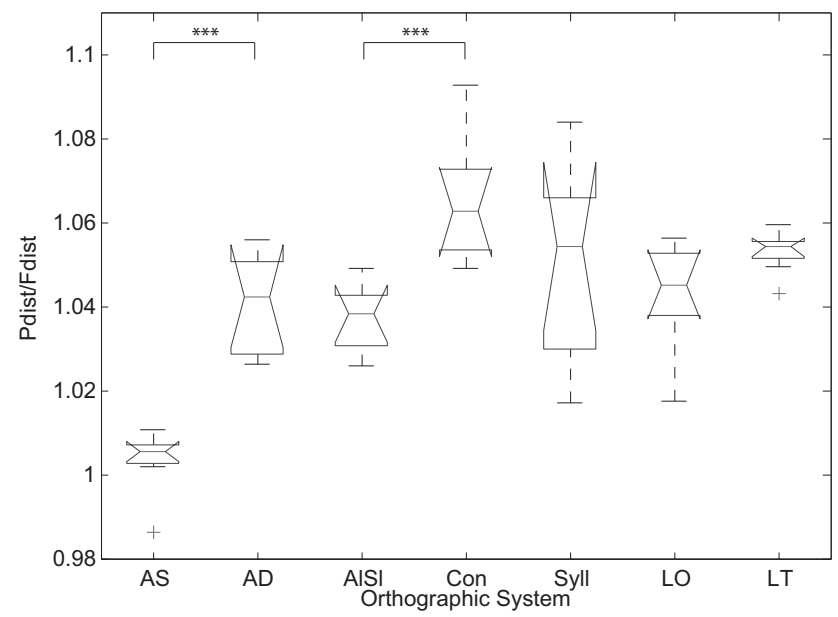

Figure 17. Pdist/Fdist displayed by trained networks (90\% accuracy on reading comprehension task) recorded at time step 12 of reading task. $\mathrm{AS}=$ alphabetic shallow; $\mathrm{AD}=$ alphabetic deep; AlS1 = alphasyllabic; Con $=$ consonantal; Syll $=$ syllabic; LO = logographic semantically opaque; $\mathrm{LT}=$ logographic semantically transparent.

a lower Pdist/Fdist ratio for alphabetic shallow networks compared with alphabetic deep $(M=-0.037, S D=0.010, t(14)=-7.581$, $p<.001)$, and for alphasyllabic networks compared with consonantal networks $(M=-0.028, S D=0.011, t(14)=-4.627, p<$ .001 ). No other comparison proved significant $(|\mathrm{t}|<2.02, p\rangle$ $.05)$.

Literacy effects on spoken language processing. Pdist/Fdist ratios were also examined at time step 12 of phonological retention trials performed by networks able to map from orthography to phonology and semantics accurately for $90 \%$ of words in the training corpus. Figure 18 presents the Pdist/Fdist ratio displayed by each system on retention tasks averaged across all items and simulations. A one-way ANOVA compared systems by simulation on this ratio revealing that systems differed in this measure, $F(6$, 49) $=7.64, \eta^{2}=0.485, p<.001$. Six two-sample $t$ tests were also performed to test differences between individual orthographic systems (Alphabetic Shallow, Alphabetic Deep; Alphabetic Deep, Alphasyllabic; Alphasyllabic, Consonantal; Consonantal, Syllabic; Syllabic, Logographic Transparent; Logographic Transparent, Logographic Opaque). These tests revealed that consonantal networks displayed a greater Pdist/Fdist ratio than syllabic networks $(M=0.012, S D=0.006, t(14)=4.194, p<.001)$, while no other test revealed a significant difference between systems $(|\mathrm{t}|<1.55$, $p>.15)$.

Discussion: Phonological effects. Cognitive neuroscientific evidence (see section Effects of literacy on representational structure) suggests that effects of literacy on phonological processing may be observed as a consequence of a restructuring of phonological processing regions used for spoken word processing or because of online activation of orthographic representations during speech processing (although see Huettig et al., 2015). Results reported in section Literacy effects on phonological processing predict that the orthography on which the reading system is trained is likely to determine the nature of the effect on processing and further that the nature of the effect observed may depend on 


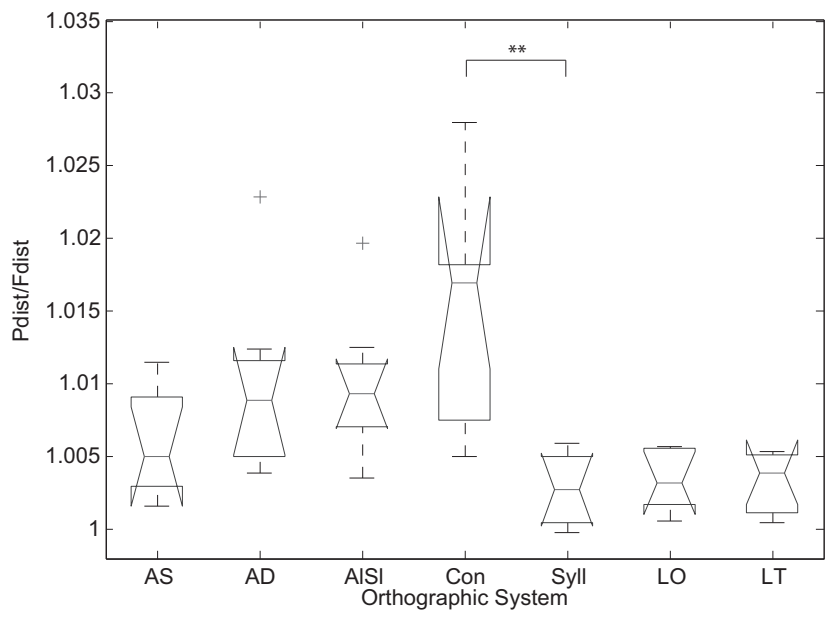

Figure 18. Pdist/Fdist displayed by trained simulations (90\% accuracy on reading comprehension and phonological decoding tasks) recorded at time step 12 of phonological retention task. $\mathrm{AS}=$ alphabetic shallow; $\mathrm{AD}=$ alphabetic deep; AlSl = alphasyllabic; Con = consonantal; Syll = syllabic; LO = logographic semantically opaque; LT = logographic semantically transparent.

whether orthographic information is also active. Within the current study, although systems displayed identical preliterate phonological processing behavior differences emerged in processing as a consequence of literacy training. Literacy training affected both the structure of the phonological representation of a word when activated by its orthographic form (online activation) and the structure of phonological representations activated in the absence of orthographic activation (phonological restructuring).

The Pdist/Fdist ratio reported above permits an examination of the extent to which systems displayed componential phonological processing. A lower ratio indicates that items that share phonemes are processed more similarly than items that simply share the same number of phonological features. We had predicted based on the existing behavioral evidence, theoretical (Psycholinguistic Grain Size Theory), and computational models (see section Effects of literacy on representational structure) that orthographic training should affect phonological processing such that processing reflects the systematic relations between orthography and phonology within the given orthographic system. Therefore, shallow alphabetic systems, in which there is one to one correspondence between graphemes and phonemes, should develop stronger componential phonemic level processing than logographic systems in which correspondence between orthography and phonology only exists at the word level. Therefore, transparent systems should display lower Pdist/Fdist ratios.

Examining phonological processing when a word's orthographic representation was also active (section Granularity of phonological processing during reading) revealed a graded effect of transparency on Pdist/Fdist ratios. This fitted with our predictions of coarser grained processing in less transparent systems. Over the course of training on all orthographies there is increasing phoneme-level processing that is a consequence of recognition of phonemes comprising the spoken forms of words. However, the extent to which this affects processing is modulated by the instantiation of this phoneme-level granular- ity in the orthographic system. This is reflected in behavioral data that shows there are early and relatively stable effects of phoneme awareness in literacy training (Alcock et al., 2010; De Jong \& Van der Leij, 1999; Hulme et al., 2005; Treiman \& Zukowski, 1991).

The simulations also reveal differences in phonological representations activated during phonological retention tasks (section Literacy effects on spoken language processing), in tasks without orthographic input. The restructuring effects observed in this analysis are, although limited, consistent with observed orthographic consistency effects reported in the literature showing that phonological processing regions become restructured as a result of literacy training (e.g., Perre et al., 2009) and, therefore, display effects of orthographic knowledge in the absence of activation of orthographic representations. This data offers an explicit description of the mechanisms that may be driving these effects.

In contrast to our predictions, logographic and syllabic systems displayed little effect of literacy on phonological processing in the absence of orthographic activation. In such systems increased phoneme overlap had little effect on the similarity of representations. These data suggest that should restructuring occur within logographic or syllabic systems it has little effect on phoneme level processing in the absence of orthographic activation. As indicated by comparisons of Figures 17 and 18, as transparency decreases systems appear to display greater variation in effects between conditions in which orthographic activation is either present or absent. A potential explanation for this observed difference is that when orthography is present there is less systematic similarity between orthography and phonology; thus, the system must learn to draw phonological representations with shared phonemes into the same representational space given distinct orthographic activation. In the absence of orthographic activation this pressure is likely to still be present in the network; thus, we see an increase in similarity of items with shared phonemes when orthographic input is absence relative to when orthographic input is present as transparency decreases.

Our simulations provide evidence in support of arguments (e.g., Dehaene et al., 2010) that orthographic effects on phonological processing can result from both phonological restructuring and online orthographic activation. Further, the simulations suggest that effects generated by restructuring are likely to be distinct from those generated by online activation especially in the case of opaque orthographies. Effects on phonological processing generated in the presence of orthographic activation are also predicted to be greater than restructuring effects. Should orthographic activation only occur at later stages of processing or require explicit or deeper processing of the phonological structure of spoken words, this may offer an explanation for why.

\section{Examining the Effects of Orthographic Transparency: Disyllabic Simulations}

Our analysis of relationships between orthographic system and phonological structure (see section The Influence of Language Structure on Orthography: Do Spoken Languages Get the Orthographic Systems They Deserve?) suggests that although some 
biases exist, orthographic system frequently varies across all dimensions of spoken language structure. However, the simulations in section Examining the Effects of Orthographic Transparency: Mono-Syllabic Simulations utilize only monosyllabic words with a large range of possible syllables. To examine the extent to which the results of our simulations across orthographic systems may be generalizable to other phonological systems, we explored whether differences in syllable structure may significantly alter our reported effects of orthographic transparency. Although other language characteristics have been proposed to influence selection of orthographic system (see section The Influence of Language Structure on Orthography: Do Spoken Languages Get the Orthographic Systems They Deserve?), the size and complexity of the syllabary varies greatly across languages and is frequently proposed as a determining factor.

In this second set of simulations, we ran exactly the same analyses as in section Examining the Effects of Orthographic Transparency: Mono-Syllabic Simulations, but on networks trained on artificial disyllabic languages with simpler syllabic structure $(\mathrm{CVCV})$. This reduced the phoneme syllable ratio by a factor of 10 from 15:500 to 15:50. In the interests of conciseness we implemented only the two extremes of the transparency spectrum namely alphabetic shallow and logographic opaque systems, in addition to a syllabic orthographic system. In the monosyllabic language of section Examining the Effects of Orthographic Transparency: Mono-Syllabic Simulations, syllabic systems were very similar to logographic trained networks because they only differed in their representation of the homophones. Implementing a syllabic orthography in a multisyllabic language ensures that graphemes change from encoding largely one-to-one mappings to one-tomany mappings and, thus, the syllabic and logographic orthographic systems should be more distinct for these simulations.

\section{Architecture}

In the disyllabic simulations reported below, the size of the phonological and orthographic layers was reduced from 50 units to 40 units per layer. This was a consequence of all words consisting of four rather than five phonemes (as used in the monosyllabic simulations). All other aspects of the model's architecture were identical to those used in monosyllabic simulations.

\section{Representations}

Semantic representations. Semantic representations were identical to those used in the monosyllabic simulations.

Phonological representations. All phonological representations were four phonemes in length, disyllabic, and of the form CVCV. The phonological layer consisted of four phoneme slots organized CVCV, with each slot 10 units in length. Replicating the monosyllabic simulations, a phoneme inventory was constructed consisting of five vowels and 10 consonants. Further, each phoneme was again encoded by a unique 10 unit phonological feature vector with $\mathrm{p}$ (active) $=0.5$. Each vowel was paired with each consonant to construct 50 unique syllables. 500 unique disyllabic words were constructed by randomly pairing syllables, while controls ensured that syllables, consonants and vowels were repeated equally within each 500 word corpus. To generate homophones a random set of 25 phonological forms were selected and duplicated, replacing 25 of the remaining 475 existing words.
Orthographic representations. Orthographic forms were generated for all words within each corpus for alphabetic shallow, syllabic, and logographic opaque systems. Each orthographic representation consisted of a 40 unit binary feature vector with $\mathrm{p}(\mathrm{ac}-$ tive) $=0.5$.

Alphabetic shallow. Alphabetic shallow representations were generated using a method almost identical to the monosyllabic simulations. The exception being that for disyllabic simulations the orthographic layer was defined in terms of four letter slots organized in a CVCV structure.

Syllabic. To generate syllabic orthographic representations a grapheme, consisting of a distinct 20 unit binary feature vector $[\mathrm{p}($ active $)=0.5]$ was created to encode each syllable. The orthographic layer consisted of two grapheme slots.

Logographic opaque. Logographic representations were created using a process almost identical to that used in monosyllabic simulations the only difference being that binary feature vectors were 40 units in length.

\section{Training}

Training of networks followed a procedure identical to that performed for monosyllabic simulations. Eight networks were trained per orthographic system, each with different randomizations of order of patterns selected, initial weight states, and trained on a distinct artificially generated corpora.

\section{Disyllabic Simulation Results}

The same analyses performed during monosyllabic simulations were also conducted during disyllabic simulations to examine effects of orthographic system on reading acquisition and the division of labor within the reading system.

Reading acquisition. At 5,000 training trial intervals, networks were tested on their ability to map from orthography to both phonology and semantics for all words in the training corpus. Figure 19A presents performance on orthographic to phonological mappings for networks trained on each orthographic system averaged over eight simulation runs per system. A one-way ANOVA compared across orthographic systems the number of training trials required to achieve $90 \%$ accuracy on the phonological decoding task revealing a significant effect of orthographic system, $F(2,23)=622.5, \eta^{2}=0.98, p<.001$. Two two-sample $t$ tests showed that networks trained on alphabetic shallow scripts required fewer training trials than networks trained on syllabic scripts to reach equivalent levels of phonological decoding ability $(M=-12,500, S D=2,216, t(14)=-11, p<.001)$, while networks trained on syllabic scripts required fewer training trials than those trained on logographic scripts $(M=-58,750, S D=$ $4,955, t(14)=-23.7, p<.001)$.

Figure 19B shows the mean accuracy of networks reading comprehension ability across literacy training. A one-way ANOVA revealed a significant effect of orthographic system on the number of training trials required to reach $90 \%$ accuracy on this task, $F(2,23)=46.12, \eta^{2}=0.81, p<.001$. Again, two two-way $t$ tests were performed which showed that networks trained on alphabetic scripts required fewer training trials to reach $90 \%$ accuracy than networks trained on syllabic scripts $(M=-8,750, S D=6,748, t(14)=-2.593, p=.021)$, whereas 
A

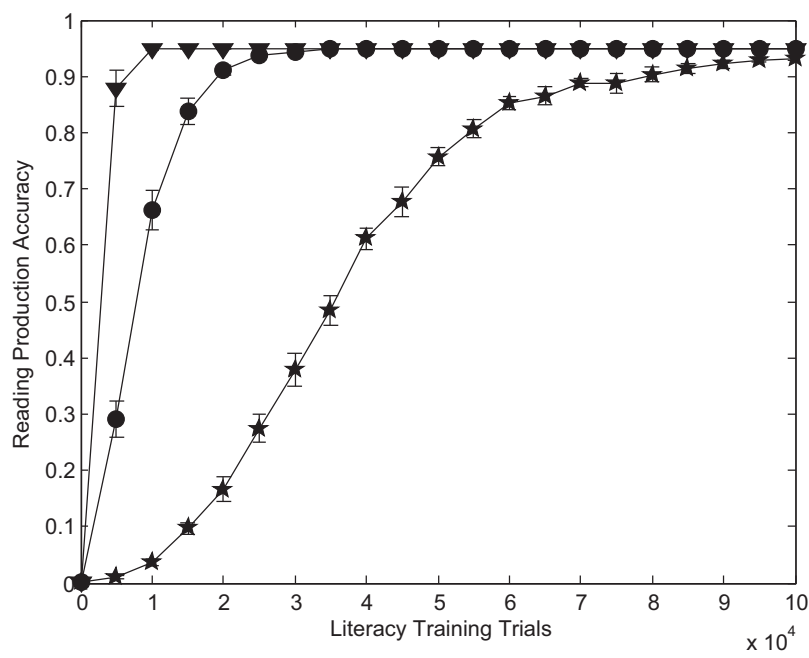

B

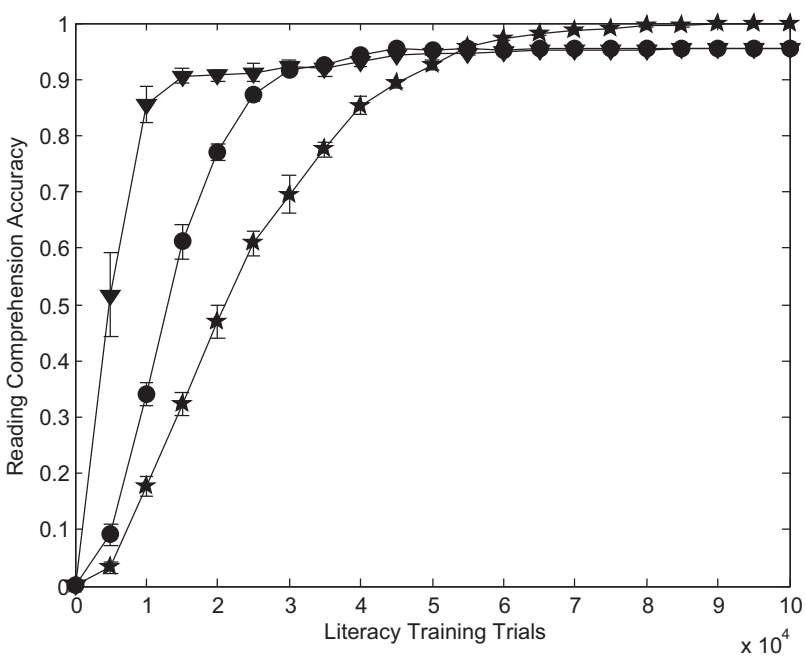

C

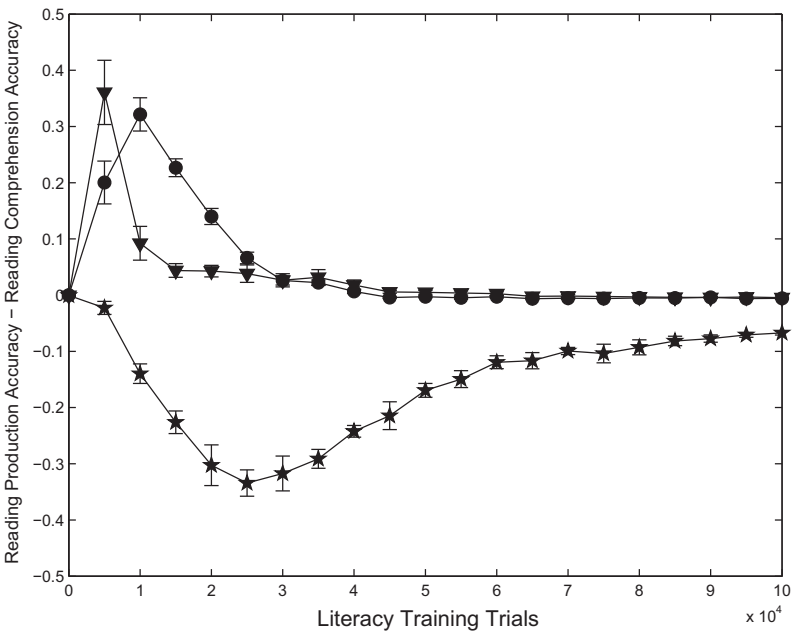

$\nabla$ Alphabetic Shallow Syllabic

$\star \quad$ Logographic Opaque

Figure 19. Literacy acquisition for networks trained on disyllabic language. (A) Phonological decoding accuracy; (B) reading comprehension accuracy; and (C) difference between phonological decoding accuracy and reading comprehension accuracy.

networks trained on syllabic scripts required fewer training trials to reach equal proficiency to networks trained on logographic scripts $(M=-18,125, S D=2,630, t(14)=-13.78, p<.001)$.

A comparison of the learning trajectories for phonological decoding and reading comprehension was also performed. The difference between phonological decoding accuracy and reading comprehension accuracy is plotted for each orthographic system across literacy training in Figure 19C. The summed difference over the initial 250,000 training trials was compared using a one-way ANOVA revealing an effect of orthographic system, $F(2,23)=$ $1,622, \eta^{2}=0.99, p<.001$. Two two-way $t$ tests revealed that decoding accuracy exceeded reading comprehension for syllabic systems to a greater extent than alphabetic shallow systems $(M=-0.284, S D=0.17, t(14)=-3.437, p=.004)$ and logographic systems $(M=4.682, S D=0.18, t(14)=51.750, p<$ $.001)$.
In summary, networks trained on alphabetic and logographic scripts produce qualitatively the same behavior during acquisition irrespective of whether the language is mono-syllabic with complex syllable structure or disyllabic with simple syllable structure. Alphabetic trained networks rapidly learn orthographic to phonology mappings, followed by orthographic to semantic mappings which are learnt quicker than logographic networks, potentially assisted by their superior phonological decoding ability. Whereas logographic systems learn orthographic to semantic mappings at a faster rate than orthographic to phonological mappings. By contrast the behavior of syllabic trained networks differs qualitatively between simulations run on monosyllabic versus disyllabic languages. When trained on monosyllabic words the networks displayed behavior similar to that of logographic networks, whereas the behavior when trained on disyllabic words was qualitatively similar to an alphabetic trained network, though with slightly 
delayed acquisition. Our explanation for this change is discussed below.

Division of labor. We repeated the division of labor analyses performed for monosyllabic simulations for networks trained on disyllabic input, examining effects of orthographic structure on the flow of activation across direct and indirect paths during reading comprehension and phonological decoding.

Figure 20A presents, for each orthographic system, the log ratio of activation entering the phonological layer via the indirect (orthography $\rightarrow$ semantics $\rightarrow$ phonology) path and activation entering the phonological layer via the direct (orthography $\rightarrow$ phonology) path during a reading task, performed at each stage of literacy training. The same measure was also calculated at each time step of a reading trial performed by networks once they attained $90 \%$ accuracy on both phonological decoding and reading comprehension tasks. To test for an effect of orthographic system on the distribution of labor across processing paths during phonological decoding the summed ratio across all time steps within a reading trial was calculated. A one-way ANOVA performed on this measure revealed an effect of orthographic system, $F(2,23)=8.29$, $\eta^{2}=0.44, p=.002$. Two two-way $t$ tests further revealed no difference between networks trained on alphabetic shallow or syllabic systems $(M=-2.842, S D=3.87, t(14)=-1.468, p=$ $.164)$, however networks trained on logographic systems displayed a greater indirect route bias than networks trained on syllabic systems $(M=-4.892, S D=4.33, t(14)=-2.261, p=.040)$.

The same analyses were performed on the ratio of activation entering the semantic layer from indirect (orthography $\rightarrow$ phonology $\rightarrow$ semantics) and direct (orthography $\rightarrow$ semantics) paths. Figure 20B displays the mean log ratio of activation, for networks trained on each orthographic system, across literacy training. The sum of the ratio calculated at each time step within a reading trial for networks that have attained $90 \%$ accuracy on both reading comprehension and phonological decoding tasks was calculated across reading trials and again submitted to a one-way ANOVA to test for effects of orthographic system revealing a significant effect, $F(2,23)=361.44, \eta^{2}=0.97, p<.001$. Further, two two-way $t$ tests showed a greater indirect bias for networks trained on alphabetic shallow systems than syllabic systems $(M=17.333$, $S D=3.34, t(14)=10.37, p<.001)$, while networks trained on syllabic systems displayed a greater indirect bias than networks trained on logographic systems $(M=25.176, S D=3.01, t(14)=$ $16.744, p<.001)$.

To summarize the alphabetic and syllabic networks displayed qualitatively similar patterns of behavior in the distribution of labor across processing paths with greater activation passing along the orthographic to phonological path and further indirectly to the semantic processing layer particularly at early stages of literacy training. By contrast at earlier stages of literacy training logographic trained networks displayed greater activation along the orthographic to semantics path and the further indirect route to the phonological layer.

\section{Disyllabic Simulations Summary}

The disyllabic language simulations largely replicate effects of orthographic transparency on both acquisition and processing observed in simulations performed on monosyllabic languages with larger, more complex syllable inventories. The exception was for networks trained on syllabic orthographies. Moving from a monosyllabic language with a large syllable inventory to use of a disyllabic language possessing a small syllable inventory dramatically altered the mappings of the syllabic system, increasing the granularity from operating largely at the word level (one-toone mappings) to a componential sublexical system operating at the syllable (one-to-many mappings) level. Although we acknowledge that a weakness of these simulations is that representations of CV syllables are not shared across locations within the word, this does not impede the model's ability to generate the properties observed, in distinction to the results of the first simulations of the
A

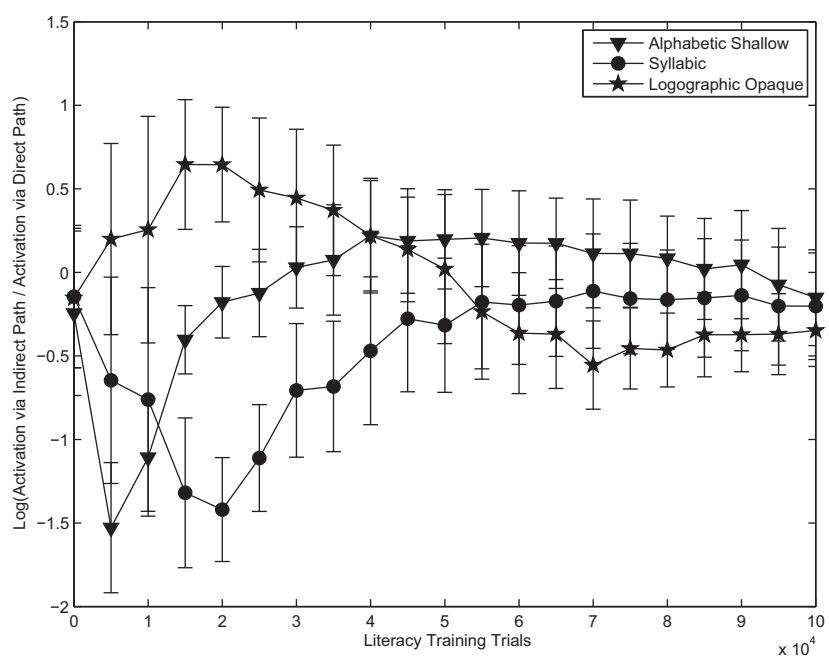

B

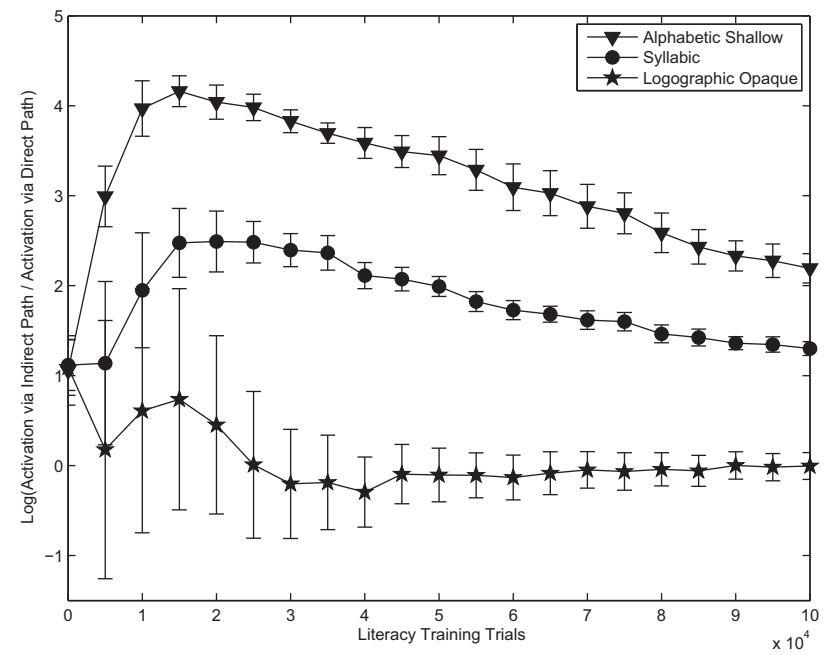

Figure 20. Log ratio of activation entering phonological layer (A), semantic layer (B) via indirect path/via indirect path during reading trials across training averaged over all items and simulations (B). 
model trained on a language comprising a larger number of syllables. Thus, it is the differentiation in the componential structure of words that the model can harness that leads to the contrast in results between monosyllabic and disyllabic simulations. Our combined results across monosyllabic and disyllabic simulations suggest that for orthographic systems that possess regular componential mappings between phonology and orthography at the syllable level or lower, phonological decoding precedes comprehension. Further, increased phonological transparency leads to increased rates of phonological decoding acquisition, which in turn leads to accelerated acquisition of reading comprehension abilities and results in networks quicker to achieve equilibrium on the distribution of labor across processing paths. Phonological transparency also leads to, at least at earlier stages of literacy training, greater reliance on initial activation of phonological representations, faster activation of phonological representations, and greater activation entering semantic processing layers indirect phonological path. Syllabic networks trained on the disyllabic language display a pattern of behavior qualitatively similar to alphabetic shallow networks yet slightly delayed in acquisition.

\section{General Discussion}

This study examined the scope of the triangle model of reading as a framework able to test reading in each of the world's major orthographic systems. Below we summarize the range of key empirical findings such a universal model of reading is able to replicate and describe the computational mechanisms within the model that generate these behavioral effects, while also discussing insights that can be derived from limitations of the current implementation.

Our model provides an explicit description of how contrasts in processing can emerge as a consequence of differences in the statistical structure of the reading environment imposed by alternative orthographic systems. Previous experimental, imaging, and computational attempts to isolate the effects of orthographic transparency have been limited by linguistic factors such as differences in semantic or phonological structure, or sociocultural factors such as language exposure, teaching methods or student motivation. By assuming that reading across orthographic systems is supported by the same underlying architecture and statistical learning mechanisms we can isolate the effects of orthographic transparency by manipulating the extent to which phonological or semantic structure is encoded within the orthography while holding phonological and semantic structure fixed. This approach allows us to demonstrate how orthographic transparency alone affects processing across literacy development.

On the issue of acquisition our study replicates and offers explicit explanation for behavioral findings that show that phonological transparency aids phonological decoding acquisition. Because of the componential phonological information encoded in the orthography, networks trained on orthographies with sublexical regularities reached proficiency in phonological decoding before networks trained on logographic systems. The modeling further offers an empirically verifiable prediction of a positive effect of semantic transparency on decoding acquisition, with a marginal difference observed between networks trained on logographic opaque compared with logographic semantically transparent systems. Our simulations also predict a positive influence of trans- parency on reading comprehension acquisition. However, the few extant studies systematically examining the effects of transparency on reading comprehension suggest that transparency may reduce reading comprehension acquisition rates (see Seidenberg, 2011, 2013, for review). Further behavioral studies are required to thoroughly test this relationship, however should such a relationship be found to exist our modeling constrains explanations to factors beyond the level of monosyllabic or disyllabic transparency to explanations relating to immaturity of phonological and semantic knowledge of a language before literacy training (Chang \& Monaghan, 2019).

The current study also provides an explicit explanation of how processing differences can emerge as a consequence of orthographic transparency. We demonstrate how differences in activation of dorsal and ventral paths of the reading network both across development and in mature systems can arise as emergent consequences of differences in orthographic transparency. Networks trained on orthographic systems that encoded sublexical phonological structure displayed greater activation entering semantic processing regions via indirect paths (orthography to semantics via phonology) during word reading comprehension relative to activation entering via direct paths (orthography to semantics) compared with logographic systems. By contrast, networks trained on alphabetic and alphasyllabic systems displayed a greater level of activation entering phonological processing regions via direct paths (orthography to phonology) relative to activation entering such regions via indirect paths (orthography to phonology via semantics) during phonological decoding compared with networks trained on consonantal and logographic systems. Our simulations replicate and offer explanation for previous neuroimaging results (e.g., Kiyosawa et al., 1995; Paulesu et al., 2000; Rueckl et al., 2015; Thuy et al., 2004) and generate the as yet untested prediction that alphasyllabic systems should result in a dorsal bias during reading comprehension in processing and also that differences in orthographic transparency should lead to distinctions, at least at early stages of reading acquisition, in the time-course of activation of phonological and semantic information within the reading system (see also online Supplemental Materials Section B).

Finally, the current work indicates that should such an interactive activation architecture as the triangle model support reading, then effects of orthographic transparency should be observed on phonological (and semantic) processing irrespective of whether orthographic information is active. ${ }^{11}$ Our simulations demonstrate that learning mappings between orthography and phonology affects processing in phonological processing networks with effects on phonological representations modulated both by the manner in which phonological information is encoded in the orthography (Brennan et al., 2013) as well as the presence or absence of orthographic activation during processing (Dehaene et al., 2010).

In a theoretical position piece, Frost (2012) argued that a universal theory of reading should isolate what is invariant in orthographic processing across systems. This should entail being able to describe what characterizes human orthographic systems and the

\footnotetext{
${ }^{11}$ Similarly, the model generates novel predictions with semantic processing also shown to be modulated by learning mappings between orthography and semantics when sublexical semantic information is embedded within the orthography (see online Supplemental Materials Section C).
} 
cognitive system that supports them. This set of universals should be small, general, and abstract to fit all orthographic systems. Our study examines the viability of the triangle model of reading as such a universal model and moves us closer toward isolating how orthographic structure may influence the reading process providing us with a baseline as to the initial biases a given system brings to bear on acquisition. By building in further language specific features to the model we can explore how each feature specifically affects processing. Beginning with our initial investigation at this level of abstraction of orthographic properties, this enables us to isolate then the contribution of unique language-specific features to reading development within that language, independent of the computational properties of the mapping induced by the general orthographic system itself. Having outlined the successes of the current implementation we will next examine what can be learnt from its limitations.

The orthographic system that proved most difficult to implement given the limitations of our computational approach was the consonantal system. Because of the underspecification of the orthography, consonantal systems generate a larger number of homographs. Without a top-down influence of semantic context the model was unable to distinguish between such items. This is not an issue for the broad theoretical framework, however it does place limitations on the validity of our results for consonantal systems, as the increased importance of preactivated semantic information in consonantal systems from contextual information during reading is likely to have significant implications for the processing dynamics within the reading system. Exploring such an influence of preactivated semantic information on the dynamics of processing in a consonantal system is a potential line of future investigation that could be explored using the modeling framework presented in this article (see Chang et al., 2019).

In addition, processing in the model diverged somewhat from neuroimaging studies for the syllabic system. For example, we know that an individual reading Japanese in Kana (syllabic) displays increased activation of the dorsal path compared with when they are processing Kanji (logographic). Although we observed a modulation of dorsal versus ventral processing as a function of transparency (as reflected in the division of labor analyses) this was not observed for syllabic systems when trained on monosyllabic languages. Further, Asfaha et al. (2009) provided evidence to suggest that some syllabic systems may lead to faster decoding abilities than alphabetic systems; however, our modeling results showed slower rates of acquisition for syllabic systems when trained on both mono-syllabic and disyllabic languages.

We believe there are two factors that likely underlie these contrasting results. In the monosyllabic simulations, all that defines differences between syllabic and logographic systems is the set of 25 homophones. Therefore, there is minimal difference between the complexity of learning an orthographic representation for every syllable in the language, opposed to learning a representation individually for every word in the language. The syllabic structure of Japanese however consists of approximately 100 distinct phonological units for each of which in a syllabic system there will be a distinct orthographic representation. Therefore, there is likely to be a significant decoding acquisition advantage in learning a transparent orthographic form of Japanese as only 100 distinct units are required to be learnt to decode all words in the language. As is demonstrated by the disyllabic simulations in a multisyllabic version of the current model, syllabic networks display behavior closer to that of alphabetic and alphasyllabic systems than logographic systems both in terms of literacy acquisition rate and the division of labor, bringing behavior closer to empirical findings when the phonological structure of the language is more closely approximated. Critical to these predictions, however, is likely to be the syllabic diversity of the language, for example encoding at the phoneme-level may prove more efficient in a language such as English in which there are approximately 11,492 different syllables (count derived from CELEX English Database; Baayen, Pipenbrock, \& Gulikers, 1995), compared with a language such as Japanese in which Kana scripts can represent Japanese morae with 51 symbols (Seeley, 1991). It remains an empirical question whether such properties of a language can lead to a decoding advantage for syllabic systems over alphabetic systems as has been observed (Asfaha et al., 2009).

A second factor that may influence predictions for syllabic systems are assumptions that the current model shares with many existing computational models of reading (e.g., Coltheart et al., 2001; Harm \& Seidenberg, 1999, 2004; Houghton \& Zorzi, 2003) regarding the structure and acquisition of phonological representations. Within such a class of models, phonology is represented in terms of phonological properties in which phoneme boundaries are clearly defined and variation of phonemes within types is minimal and independent of context. We know, however, that the natural speech signal is noisy and endemic with features such as coarticulation, elision, and reductions. Such features of the input are likely to have profound consequences for the emergent structure of phonological representations that are not currently captured within existing models of reading. A large body of empirical data from phonological awareness studies (see Morais \& Kolinsky, 2001) indicates that literacy in alphabetic systems significantly alters at least explicit awareness of subsyllabic phonological structure within the speech signal. Capturing an accurate depiction of the structure of emergent phonological representations is likely to greatly enhance the accuracy of predictions regarding the influence of transparency on reading acquisition and the impact of literacy acquisition on phonological processing. In Smith et al.'s (2014) model of literacy effects on phonological processing, the introduction of noise in the phonological representations in the model emphasized differences in phonological granularity relating to literacy. We anticipate that implementing noise in the environment of the current model would also elaborate differences between orthographic systems in the model's representation of phonology and semantics. Both rates of acquisition and effects of literacy on phonological processing predicted by the model are likely to alter significantly should emergent phonological representations not contain the fine grained phonetic detail present in natural language processing. One possible avenue for exploring such questions may be offered through development of models such as those used in Sibley, Kello, Plaut, and Elman (2008) that encode words from sequences of letters or sequences of phonemes. Such sequential models avoid the disadvantages of slot-based coding, but they increase the difficulty of determining how the model constructs mappings between representations.

As the typological results presented in section The Influence of Language Structure on Orthography: Do Spoken Languages Get the Orthographic Systems They Deserve? demonstrate, properties of the spoken language are somewhat related to specific ortho- 
graphic systems. Though there was no statistical effect of consonant or vowel inventory size, syllable structure, and tone system were predictors of orthographic system. However, geographical location was a far better predictor than a combination of all these phonological properties of languages, suggesting that orthographic system selection is partially a consequence of cultural or social mores, and for nearly any point in the phonological feature space, there exists a language associated with each orthographic system.

Regardless of the extent to which languages are matched to an optimal orthographic system, with our mono-syllabic and disyllabic simulations we have shown that phonological language properties (such as size of phoneme and syllable inventories) may affect the efficiency with which an orthographic system is learnt and processed. Although our simulations generate many of the prominent behavioral and neural phenomena proposed to originate from variations in orthographic transparency, many further subtle modulations of relationships likely exist beyond the scope of the current article. The framework used here however offers the ability to build in specific language characteristics to explore their potential effects. For example, future research using this modeling framework can address how a highly constrained root-derived language such as Hebrew affect observed differences in behavior between alphabetic and consonantal systems, or how morphological complexity might affect processing of distinct orthographic representations, or explore the effect context will have on semantic processing across orthographic systems.

Our investigation explored the scope of the triangle model of reading as a universal framework for supporting reading and capturing the effects of orthographic transparency on reading acquisition and processing more broadly. Each orthographic system is partially a product of the combined evolution within semantic, phonological, and orthographic structure of a language (Frost, 2012; Seidenberg, 2011, see section The Influence of Language Structure on Orthography: Do Spoken Languages Get the Orthographic Systems They Deserve?). However, we believe if we are to isolate the effects of orthographic transparency then computational modeling at such a level of abstraction provides the only means to do so. Our study demonstrates that the modeling framework presented here is able to support reading across all of the world's major orthographic systems, and further provides an explicit description for how a broad range of properties common to individual categories of orthographic system (e.g., faster phonological decoding acquisition and increased dorsal path processing bias as a consequence of increased phonological transparency) emerge from constraints placed on the statistics of the learning environment by properties common to the orthographic system. As Frost (2012) argues, "if the model indeed picks up the statistical regularities of the language and the expected reading behavior emerges it most probably reflects the actual learning procedures of readers." Here we have provided a computational investigation of this suggested approach: determining the role of the statistical regularities in the orthography on a model's acquisition and processing of reading production and comprehension. The level of abstraction with which we have begun this approach allows us to isolate the factors driving distinctions in behavior without linguistic or sociocultural confounds that have confounded previous behavioral and imaging studies, highlighting how computational constraints emerge from the nature of the orthographic system itself.
The tasks simulated in this article have focused on reading fluency and reading comprehension tasks for different orthographies. However, the simulations also have potential to indicate how lexical decision may be affected by orthography types. In Plaut (1997) and Chang et al. (2019) lexical decision is implemented in terms of the distance between the model's production of a semantic representation and a plausible semantic representation of a word in the language. If this representation exceeds a threshold-so is far from all plausible word representations-then the model is taken to make a decision that the orthographic input is a nonword. It is certainly the case that lexical decision responses relate more closely to concreteness and imageability measures than word naming, which indicate a strong semantic engagement for lexical decision. Taking this approach to lexical decision in the model would then mean that lexical decision could be simulated for all orthographic types, and could generate predictions for lexical decision responses - as well as word naming responses-in terms of the extent to which the semantic representations of the model are engaged more or less effectively by orthographic input. For example, one prediction would be that, because semantics is activated to a greater degree indirectly via the orthography to phonology to semantics pathway in an alphabetic than for a logographic orthographic system, one would expect that processing associated with the orthography to phonology pathway will have a greater influence in lexical decision behavior (such as phonological neighborhood size, or regularity) for alphabetic than logographic orthographies.

Our focus has been on determining the effect of different orthographic systems on processing of the reading architecture within the triangle model framework. Our work provides a foundation for future explorations that take into account, with greater detail, the natural variation found across the world's languages and orthographic systems. For instance, controlling the set of phonological and semantic representations glosses over the extent to which languages may contain homonymy that could have an important moderating effect on the optimality of different orthographic systems. Furthermore, phonological properties of languages can also affect the role of the orthographic system-we have tested two different syllabic structures in the current sets of simulations, but the distributions of phoneme inventories and syllabaries, as demonstrated in our survey of languages in section The Influence of Language Structure on Orthography: Do Spoken Languages Get the Orthographic Systems They Deserve?, are substantial (though apparently not neatly related to the orthographic system used by the linguistic community). A further important variant across orthographic systems, that is not yet captured in our simulations, is the visual complexity of the signs. For instance, Latin alphabets typically contain only two or three strokes per character, but Chinese contains an average of 10 closely confined strokes (Tsai, 1996). Visual complexity affects reading efficiency (Pelli, Burns, Farell, \& Moore-Page, 2006) and has been shown to influence reading acquisition (Nag, 2007; Nag, Snowling, Quinlan, \& Hulme, 2014). Comprehensive simulations of orthographic types would require capturing the visual characteristics of orthographic systems as well as the phonological variation found across languages.

Yet, the model has demonstrated profound computational distinctions evident in our implementation of different orthographies, both in terms of acquisition rate, division of labor across pathways 
in the reading system, and effects on the very representations themselves in phonology and semantics. We contend that adequate and generalizable models of reading ought to take into account this diversity in the world's orthographic systems, before generalizations can be made to the likely neural implementation and behavioral consequences that are likely to be observed across the world's languages. We have shown that the triangle model is able to provide a foundation for such an approach, and future implementations will usefully extend our approach to capture a wider range of language characteristics in reflecting the full range of reading behaviors observable around the globe.

\section{References}

Abu-Rabia, S., \& Taha, H. (2006). Reading in Arabic orthography: Characteristics, research findings, and assessment. In R. M. Joshi \& P. G. Aaron (Eds.), Handbook of orthography and literacy (pp. 321-338). Mahwah, NJ: Erlbaum.

Adrián, J. A., Alegria, J., \& Morais, J. (1995). Metaphonological abilities of Spanish illiterate adults. International Journal of Psychology, 30, 329-351. http://dx.doi.org/10.1080/00207599508246574

Alcock, K. J., Ngorosho, D., Deus, C., \& Jukes, M. C. H. (2010). We don't have language at our house: Disentangling the relationship between phonological awareness, schooling, and literacy. British Journal of Educational Psychology, 80, 55-76. http://dx.doi.org/10.1348/0007 09909X424411

Araújo, S., Fernandes, T., \& Huettig, F. (2019). Learning to read facilitates the retrieval of phonological representations in rapid automatized naming: Evidence from unschooled illiterate, ex-illiterate, and schooled literate adults. Developmental Science, 22, e12783. http://dx.doi.org/10 $1111 /$ desc. 12783

Aronoff, M., Berg, K., \& Heyer, V. (2016). Some implications of English spelling for morphological processing. The Mental Lexicon, 11, 164185. http://dx.doi.org/10.1075/ml.11.2.01aro

Asfaha, Y. M., Kurvers, J., \& Kroon, S. (2009). Grain size in script and teaching: Literacy acquisition in Ge'ez and Latin. Applied Psycholinguistics, 30, 709-724. http://dx.doi.org/10.1017/S0142716409990087

Baayen, R. H., Pipenbrock, R., \& Gulikers, L. (1995). The CELEX Lexical database [CD-ROM]. Philadelphia: Linguistic Data Consortium, University of Pennsylvania.

Bar-Kochva, I., \& Breznitz, Z. (2014). Reading scripts that differ in orthographic transparency: A within-participant-and-language investigation of underlying skills. Journal of Experimental Child Psychology, 121, 12-27. http://dx.doi.org/10.1016/j.jecp.2013.07.011

Behme, C. (2012). Can evolution provide perfectly optimal solutions for a universal model of reading? Behavioral and Brain Sciences, 35, 279280. http://dx.doi.org/10.1017/S0140525X12000015

Beveridge, M. E., \& Bak, T. H. (2012). Beyond one-way streets: The interaction of phonology, morphology, and culture with orthography. Behavioral and Brain Sciences, 35, 280-281. http://dx.doi.org/10.1017/ S0140525X12000143

Bolger, D. J., Perfetti, C. A., \& Schneider, W. (2005). Cross-cultural effect on the brain revisited: Universal structures plus writing system variation. Human Brain Mapping, 25, 92-104. http://dx.doi.org/10.1002/hbm .20124

Brennan, C., Cao, F., Pedroarena-Leal, N., McNorgan, C., \& Booth, J. R. (2013). Reading acquisition reorganizes the phonological awareness network only in alphabetic writing systems. Human Brain Mapping, 34, 3354-3368. http://dx.doi.org/10.1002/hbm.22147

Bruck, M., Genesee, F., \& Caravolas, M. (1997). A cross-linguistic study of early literacy acquisition. In B. A. Blachmann (Ed.), Foundations of reading acquisition and dyslexia: Implications for early intervention (pp. 145-162). Oxford, UK: Routledge.
Buchweitz, A., Mason, R. A., Hasegawa, M., \& Just, M. A. (2009). Japanese and English sentence reading comprehension and writing systems: An fMRI study of first and second language effects on brain activation. Bilingualism: Language and Cognition, 12, 141-151. http:// dx.doi.org/10.1017/S1366728908003970

Chang, Y.-N., \& Monaghan, P. (2019). Quantity and diversity of preliteracy language exposure both affect literacy development: Evidence from a computational model of reading. Scientific Studies of Reading, 23, 235-253. http://dx.doi.org/10.1080/10888438.2018.1529177

Chang, Y. N., Monaghan, P., \& Welbourne, S. (2019). A computational model of reading across development: Effects of literacy onset on language processing. Journal of Memory and Language, 108, 104025. http://dx.doi.org/10.1016/j.jml.2019.05.003

Chang, Y. N., Welbourne, S., \& Lee, C. Y. (2016). Exploring orthographic neighborhood size effects in a computational model of Chinese character naming. Cognitive Psychology, 91, 1-23. http://dx.doi.org/10.1016/j cogpsych.2016.09.001

Cheung, H., \& Chen, H. C. (2004). Early orthographic experience modifies both phonological awareness and on-line speech processing. Language and Cognitive Processes, 19, 1-28. http://dx.doi.org/10.1080/ 01690960344000071

Cheung, H., \& Ng, L. (2003). Chinese reading development in some major Chinese societies: An introduction. In C. McBride-Chang \& H. Chen (Eds.), Reading development in Chinese children (pp. 3-17). Westport, CT: Praeger.

Chomsky, N. (1970). Phonology and reading. In H. Levin \& J. P. Williams (Eds.), Basic studies in reading (pp. 3-18). New York, NY: Basic Books.

Chyl, K., Kossowski, B., Dębska, A., Łuniewska, M., Banaszkiewicz, A., Żelechowska, A., . . . Jednoróg, K. (2018). Prereader to beginning reader: Changes induced by reading acquisition in print and speech brain networks. Journal of Child Psychology and Psychiatry, 59, 76-87. http://dx.doi.org/10.1111/jcpp.12774

Cohen, L., Dehaene, S., Vinckier, F., Jobert, A., \& Montavont, A. (2008). Reading normal and degraded words: Contribution of the dorsal and ventral visual pathways. Neuroimage, 40, 353e366.

Cohen-Mimran, R. (2009). The contribution of language skills to reading fluency: A comparison of two orthographies for Hebrew. Journal of Child Language, 36, 657-672. http://dx.doi.org/10.1017/S0305000 908009148

Coltheart, M., Rastle, K., Perry, C., Langdon, R., \& Ziegler, J. (2001). DRC: A dual route cascaded model of visual word recognition and reading aloud. Psychological Review, 108, 204-256. http://dx.doi.org/ 10.1037/0033-295X.108.1.204

Comrie, B. (2013). Writing systems. In M. S. Dryer \& M. Haspelmath (Eds.) The world atlas of language structures online. Leipzig, Germany: Max Planck Institute for Evolutionary Anthropology. Retrieved from http://wals.info/chapter/141

Cummine, J., Dai, W., Borowsky, R., Gould, L., Rollans, C., \& Boliek, C. (2015). Investigating the ventral-lexical, dorsal- sublexical model of basic reading processes using diffusion tensor imaging. Brain Structure \& Function, 220, 445e 455

Das, T., Padakannaya, P., Pugh, K. R., \& Singh, N. C. (2011). Neuroimaging reveals dual routes to reading in simultaneous proficient readers of two orthographies. NeuroImage, 54, 1476-1487. http://dx.doi.org/10 .1016/j.neuroimage.2010.09.022

De Gelder, B. D., \& Vroomen, J. (1992). Auditory and visual speech perception in alphabetic and non-alphabetic Chinese-Dutch bilinguals. In R. J. Harris (Ed.), Cognitive processing in bilinguals (pp. 413-426). Amsterdam, the Netherlands: Elsevier. http://dx.doi.org/10.1016/S01664115(08)61508-3

Dehaene, S. (2009). Reading in the brain: The new science of how we read. New York, NY: Penguin. 
Dehaene, S., Pegado, F., Braga, L. W., Ventura, P., Nunes Filho, G., Jobert, A., . . . Cohen, L. (2010). How learning to read changes the cortical networks for vision and language. Science, 330, 1359-1364. http://dx .doi.org/10.1126/science.1194140

Dehaene-Lambertz, G., Montavont, A., Jobert, A., Allirol, L., Dubois, J., Hertz-Pannier, L., \& Dehaene, S. (2010). Language or music, mother or Mozart? Structural and environmental influences on infants' language networks. Brain and Language, 114, 53-65. http://dx.doi.org/10.1016/ j.bandl.2009.09.003

de Jong, P. F., \& van der Leij, A. (1999). Specific contributions of phonological abilities to early reading acquisition: Results from a Dutch latent variable longitudinal study. Journal of Educational Psychology, 91, 450-476. http://dx.doi.org/10.1037/0022-0663.91.3.450

Devauchelle, A. D., Oppenheim, C., Rizzi, L., Dehaene, S., \& Pallier, C. (2009). Sentence syntax and content in the human temporal lobe: An fMRI adaptation study in auditory and visual modalities. Journal of Cognitive Neuroscience, 21, 1000-1012. http://dx.doi.org/10.1162/jocn .2009 .21070

Dilkina, K., McClelland, J. L., \& Plaut, D. C. (2008). A single-system account of semantic and lexical deficits in five semantic dementia patients. Cognitive Neuropsychology, 25, 136-164. http://dx.doi.org/10 $.1080 / 02643290701723948$

Dilkina, K., McClelland, J. L., \& Plaut, D. C. (2010). Are there mental lexicons? The role of semantics in lexical decision. Brain Research, 1365, 66-81. http://dx.doi.org/10.1016/j.brainres.2010.09.057

Dryer, M. S., \& Haspelmath, M. (Eds.). (2013). The world atlas of language structures (WALS) online. Leipzig, Germany: Max Planck Institute for Evolutionary Anthropology. Retrieved from http://wals.info Durgunoglu, A. Y. (2006). How the language's characteristics influence Turkish literacy development. In R. M. Joshi \& P. G. Aaron (Eds.), Handbook of orthography and literacy (pp. 219-230). Mahwah, NJ: Erlbaum.

Francis, W. N. (1970). Linguistics and reading: A commentary on chapters 1 to 3. In H. Levin \& J. P. Williams (Eds.), Basic studies in reading (pp. 43-56). New York, NY: Basic Books.

Frith, U., Wimmer, H., \& Landerl, K. (1998). Differences in phonological recoding in German-and English-speaking children. Scientific Studies of Reading, 2, 31-54. http://dx.doi.org/10.1207/s1532799xssr0201_2

Frost, R. (2012). Author's response: A universal approach to modeling visual word recognition and reading: Not only possible, but also inevitable. Behavioral and Brain Sciences, 35, 310-329. http://dx.doi.org/10 .1017/S0140525X12000635

Frost, R., Katz, L., \& Bentin, S. (1987). Strategies for visual word recognition and orthographical depth: A multilingual comparison. Journal of Experimental Psychology: Human Perception and Performance, 13, 104-115. http://dx.doi.org/10.1037/0096-1523.13.1.104

Gelb, I. J. (1952). A study of writing: The foundations of grammatology. Chicago, IL: University of Chicago Press.

Ghetie, I. (1978). Istoria limbii Romane literare [History of the Romanian literary language]. Bucharest: Editura Stiintifica si Enciclopedica.

Goswami, U., Gombert, J. E., \& de Barrera, L. F. (1998). Children's orthographic representations and linguistic transparency: Nonsense word reading in English, French, and Spanish. Applied Psycholinguistics, 19, 19-52. http://dx.doi.org/10.1017/S0142716400010560

Hanley, R., Masterson, J., Spencer, L., \& Evans, D. (2004). How long do the advantages of learning to read a transparent orthography last? An investigation of the reading skills and reading impairment of Welsh children at 10 years of age. Quarterly Journal of Experimental Psychology, 57, 1393-1410. http://dx.doi.org/10.1080/02724980343000819

Harm, M. W., \& Seidenberg, M. S. (1999). Phonology, reading acquisition, and dyslexia: Insights from connectionist models. Psychological Review, 106, 491-528. http://dx.doi.org/10.1037/0033-295X.106.3.491

Harm, M. W., \& Seidenberg, M. S. (2004). Computing the meanings of words in reading: Cooperative division of labor between visual and phonological processes. Psychological Review, 111, 662-720. http://dx .doi.org/10.1037/0033-295X.111.3.662

Havelock, E. A. (1982). The literate revolution in Greece and its cultural consequence. Princeton, NJ: Princeton University Press.

Hervais-Adelman, A., Kumar, U., Mishra, R. K., Tripathi, V. N., Guleria, A., Singh, J. P., . . . Huettig, F. (2019). Learning to read recycles visual cortical networks without destruction. Science Advances, 5, eaax0262. http://dx.doi.org/10.1126/sciadv.aax0262

Hirshorn, E. A., \& Fiez, J. A. (2014). Using artificial orthographies for studying cross-linguistic differences in the cognitive and neural profiles of reading. Journal of Neurolinguistics, 31, 69-85. http://dx.doi.org/10 .1016/j.jneuroling.2014.06.006

Hoonhorst, I., Medina, V., Colin, C., Markessis, E., Radeau, M., Deltenre, P., \& Serniclaes, W. (2011). Categorical perception of voicing, colors and facial expressions: A developmental study. Speech Communication, 53, 417-430. http://dx.doi.org/10.1016/j.specom.2010.11.005

Houghton, G., \& Zorzi, M. (2003). Normal and impaired spelling in a connectionist dual-route architecture. Cognitive Neuropsychology, 20, 115-162. http://dx.doi.org/10.1080/02643290242000871

Hudson, R. F., Torgesen, J. K., Lane, H. B., \& Turner, S. J. (2012). Relations among reading skills and sub-skills and text-level reading proficiency in developing readers. Reading and Writing, 25, 483-507. http://dx.doi.org/10.1007/s11145-010-9283-6

Huettig, F., Kumar, U., Mishra, R., Tripathi, V., Guleria, A., Singh, J. P., \& Eisner, F. (2015, September). The effect of learning to read on the neural systems for vision and language: A longitudinal approach with illiterate participants. Talk presented at the 19th Meeting of the European Society for Cognitive Psychology (ESCoP 2015). Paphos, Cyprus.

Huettig, F., \& Mishra, R. K. (2014). How literacy acquisition affects the illiterate mind - A critical examination of theories and evidence. Language and Linguistics Compass, 8, 401-427. http://dx.doi.org/10.1111/ lnc3.12092

Huettig, F., Singh, N., \& Mishra, R. K. (2011). Language-mediated visual orienting behavior in low and high literates. Frontiers in Psychology, 2 285. http://dx.doi.org/10.3389/fpsyg.2011.00285

Hulme, C., Snowling, M., Caravolas, M., \& Carroll, J. (2005). Phonological skills are (probably) one cause of success in learning to read: A comment on Castles and Coltheart. Scientific Studies of Reading, 9, 351-365. http://dx.doi.org/10.1207/s1532799xssr0904_2

Jobard, G., Crivello, F., \& Tzourio-Mazoyer, N. (2003). Evaluation of the dual route theory of reading: A metanalysis of 35 neuroimaging studies NeuroImage, 20, 693-712. http://dx.doi.org/10.1016/S1053-8119 (03)00343-4

Katz, L., \& Feldman, L. B. (1981). Linguistic coding in word recognition: Comparisons between a deep and a shallow orthography. In A. M Lesgold \& C. A. Perfetti (Eds.), Interactive processes in reading (pp. 85-106). Hillsdale, NJ: Erlbaum.

Kidd, J. C., Shum, K. K. M., Ho, C. S. H., \& Au, T. K. F. (2015). Phonological representations and early literacy in Chinese. Scientific Studies of Reading, 19, 89-113. http://dx.doi.org/10.1080/10888438 .2014 .938192

Kiyosawa, M., Itoh, M., Nakagawa, Y., Kobayashi, N., \& Tamai, M (1995). Effect of kanji and kana reading on cerebral blood flow patterns measured by PET. Japanese Journal of Ophthalmology, 39, 198-205.

Klima, E. S. (1972). How alphabets might reflect language. In J. F. Kavanagh \& I. G. Mattingly (Eds.), Language by ear and by eye: The relationship between speech and reading (pp. 57-80). Cambridge, MA: MIT Press.

Levy, J., Pernet, C., Treserras, S., Boulanouar, K., Aubry, F., Démonet, J. F., \& Celsis, P. (2009). Testing for the dual-route cascade reading model in the brain: An fMRI effective connectivity account of an efficient reading style. PLOS ONE, 4, e6675. http://dx.doi.org/10.1371/ journal.pone.0006675 
Levy, J., Pernet, C., Treserras, S., Boulanouar, K., Berry, I., Aubry, F., . . . Celsis, P. (2008). Piecemeal recruitment of left-lateralized brain areas during reading: A spatio-functional account. NeuroImage, 43, 581-591. http://dx.doi.org/10.1016/j.neuroimage.2008.08.008

Loureiro, C. S., Braga, L. W., Souza, L. N., Nunes Filho, G., Queiroz, E., \& Dellatolas, G. (2004). Degree of illiteracy and phonological and metaphonological skills in unschooled adults. Brain and Language, 89, 499-502. http://dx.doi.org/10.1016/j.bandl.2003.12.008

Maddieson, I. (2013a). Consonant inventories. In M. S. Dryer \& M. Haspelmath (Eds.), The world atlas of language structures online. Leipzig, Germany: Max Planck Institute for Evolutionary Anthropology. Retrieved from http://wals.info/chapter/1

Maddieson, I. (2013b). Vowel quality inventories. In M. S. Dryer \& M. Haspelmath (Eds.), The world atlas of language structures online. Leipzig, Germany: Max Planck Institute for Evolutionary Anthropology. Retrieved from http://wals.info/chapter/2

Maddieson, I. (2013c). Consonant-vowel ratio. In M. S. Dryer \& M. Haspelmath (Eds.), The world atlas of language structures online. Leipzig, Germany: Max Planck Institute for Evolutionary Anthropology. Retrieved from http://wals.info/chapter/3

Maddieson, I. (2013d). Syllable structure. In M. S. Dryer \& M. Haspelmath (Eds.), The world atlas of language structures online. Leipzig, Germany: Max Planck Institute for Evolutionary Anthropology. Retrieved from http://wals.info/chapter/12

Maddieson, I. (2013e). Tone. In M. S. Dryer \& M. Haspelmath (Eds.), The world atlas of language structures online. Leipzig, Germany: Max Planck Institute for Evolutionary Anthropology. Retrieved from http:// wals.info/chapter/13

Magnusson, A. (2017). gmt: Interface between GMT map-making software and $R$ (R package version 2.0-1). Retrieved from https://CRAN.Rproject.org/package $=$ gmt

Malins, J. G., Pugh, K. R., Buis, B., Frost, S. J., Hoeft, F., Landi, N., . . Morris, R. (2018). Individual differences in reading skill are related to trial-by-trial neural activation variability in the reading network. The Journal of Neuroscience, 38, 2981-2989. http://dx.doi.org/10.1523/ JNEUROSCI.0907-17.2018

Mitterer, H., \& Reinisch, E. (2015). Letters don't matter: No effect of orthography on the perception of conversational speech. Journal of Memory and Language, 85, 116-134. http://dx.doi.org/10.1016/j.jml 2015.08.005

Monaghan, P., \& Ellis, A. W. (2010). Modeling reading development: Cumulative, incremental learning in a computational model of word naming. Journal of Memory and Language, 63, 506-525. http://dx.doi .org/10.1016/j.jml.2010.08.003

Monaghan, P., Shillcock, R. C., Christiansen, M. H., \& Kirby, S. (2014). How arbitrary is language? Philosophical Transactions of the Royal Society of London. Series B, Biological Sciences, 369, 20130299. http:// dx.doi.org/10.1098/rstb.2013.0299

Monaghan, P., Shillcock, R., \& McDonald, S. (2004). Hemispheric asymmetries in the split-fovea model of semantic processing. Brain and Language, 88, 339-354. http://dx.doi.org/10.1016/S0093-934X(03) 00165-2

Monzalvo, K., \& Dehaene-Lambertz, G. (2013). How reading acquisition changes children's spoken language network. Brain and Language, 127, 356-365. http://dx.doi.org/10.1016/j.bandl.2013.10.009

Morais, J., Cary, L., Alegria, J., \& Bertelson, P. (1979). Does awareness of speech as a sequence of phones arise spontaneously? Cognition, 7, 323-331. http://dx.doi.org/10.1016/0010-0277(79)90020-9

Morais, J., \& Kolinsky, R. (2001). The literate mind and the universal human mind. In E. Dupoux (Ed.), Language, brain and cognitive development: Essays in Honor of Jacques Mehler (pp. 463-480). Cambridge, MA: MIT Press.

Muneaux, M., \& Ziegler, J. (2004). Locus of orthographic effects in spoken word recognition: Novel insights from the neighbour generation task.
Language and Cognitive Processes, 19, 641-660. http://dx.doi.org/10 .1080/01690960444000052

Nag, S. (2007). Early reading in Kannada: The pace of acquisition of orthographic knowledge and phonemic awareness. Journal of Research in Reading, 30, 7-22. http://dx.doi.org/10.1111/j.1467-9817.2006 .00329.x

Nag, S., Snowling, M., Quinlan, P., \& Hulme, C. (2014). Child and symbol factors in learning to read a visually complex writing system. Scientific Studies of Reading, 18, 309-324. http://dx.doi.org/10.1080/10888438 .2014.892489

Nakamura, K., Kuo, W. J., Pegado, F., Cohen, L., Tzeng, O. J., \& Dehaene, S. (2012). Universal brain systems for recognizing word shapes and handwriting gestures during reading. Proceedings of the National Academy of Sciences, 109, 20762-20767. http://dx.doi.org/10.1073/pnas .1217749109

Nation, K. (2009). Form-meaning links in the development of visual word recognition. Philosophical Transactions of the Royal Society of London Series B, Biological Sciences, 364, 3665-3674. http://dx.doi.org/10 1098/rstb.2009.0119

Nation, K., \& Cocksey, J. (2009). The relationship between knowing a word and reading it aloud in children's word reading development Journal of Experimental Child Psychology, 103, 296-308. http://dx.doi org/10.1016/j.jecp.2009.03.004

Norris, D., \& Kinoshita, S. (2012). Orthographic processing is universal; it's what you do with it that's different. Behavioral and Brain Sciences, 35, 296-297. http://dx.doi.org/10.1017/S0140525X12000106

O'Connor, C. M., Cree, G. S., \& McRae, K. (2009). Conceptual hierarchies in a flat attractor network: Dynamics of learning and computations. Cognitive Science, 33, 665-708. http://dx.doi.org/10.1111/j.1551-6709 2009.01024.x

Olson, D. R. (1988). Encyclopedia Britannica: Writing. Chicago, IL: Encyclopedia Britannica.

Pattamadilok, C., Knierim, I. N., Kawabata Duncan, K. J., \& Devlin, J. T. (2010). How does learning to read affect speech perception? The Journal of Neuroscience, 30, 8435-8444. http://dx.doi.org/10.1523/JNEUROSCI $.5791-09.2010$

Paulesu, E., McCrory, E., Fazio, F., Menoncello, L., Brunswick, N., Cappa S. F., . . Frith, U. (2000). A cultural effect on brain function. Nature Neuroscience, 3, 91-96. http://dx.doi.org/10.1038/71163

Pearlmutter, B. (1989). Learning state-space trajectories. Neural Computation, 1, 263-269. http://dx.doi.org/10.1162/neco.1989.1.2.263

Pelli, D. G., Burns, C. W., Farell, B., \& Moore-Page, D. C. (2006). Feature detection and letter identification. Vision Research, 46, 4646-4674. http://dx.doi.org/10.1016/j.visres.2006.04.023

Perfetti, C. A., Liu, Y., \& Tan, L. H. (2005). The lexical constituency model: Some implications of research on Chinese for general theories of reading. Psychological Review, 112, 43-59. http://dx.doi.org/10.1037/ 0033-295X.112.1.43

Perre, L., Pattamadilok, C., Montant, M., \& Ziegler, J. C. (2009). Orthographic effects in spoken language: On-line activation or phonologica restructuring? Brain Research, 1275, 73-80. http://dx.doi.org/10.1016/ j.brainres.2009.04.018

Perry, C., Ziegler, J. C., \& Zorzi, M. (2010). Beyond single syllables: Large-scale modeling of reading aloud with the Connectionist Dua Process $(\mathrm{CDP}++)$ model. Cognitive Psychology, 61, 106-151. http:// dx.doi.org/10.1016/j.cogpsych.2010.04.001

Petersson, K. M., Ingvar, M., \& Reis, A. (2009). Language and literacy from a cognitive neuroscience perspective. In D. Olsen \& N. Torrance (Eds.), Cambridge handbook of literacy (pp. 152-182). Cambridge: Cambridge University Press. http://dx.doi.org/10.1017/CBO97805 11609664.010

Plaut, D. C. (1997). Structure and function in the lexical system: Insights from distributed models of word reading and lexical decision. Language 
and Cognitive Processes, 12, 765-806. http://dx.doi.org/10.1080/ 016909697386682

Plaut, D. C., McClelland, J. L., Seidenberg, M. S., \& Patterson, K. (1996). Understanding normal and impaired word reading: Computational principles in quasi-regular domains. Psychological Review, 103, 56-115. http://dx.doi.org/10.1037/0033-295X.103.1.56

Price, C. J. (2010). The anatomy of language: A review of 100 fMRI studies published in 2009. Annals of the New York Academy of Sciences, 1191, 62-88. http://dx.doi.org/10.1111/j.1749-6632.2010.05444.x

Price, C. J., \& Devlin, J. T. (2011). The interactive account of ventral occipitotemporal contributions to reading. Trends in Cognitive Sciences, 15, 246-253. http://dx.doi.org/10.1016/j.tics.2011.04.001

Pugh, K. R., Mencl, W. E., Jenner, A. R., Katz, L., Frost, S. J., Lee, J. R., ... Shaywitz, B. A. (2000). Functional neuroimaging studies of reading and reading disability (developmental dyslexia). Mental Retardation and Developmental Disabilities Research Reviews, 6, 207-213. http://dx.doi .org/10.1002/1098-2779(2000)6:3<207::AID-MRDD8>3.0.CO;2-P

Pugh, K. R., Mencl, W. E., Jenner, A. R., Katz, L., Frost, S. J., Lee, J. R., . . Shaywitz, B. A. (2001). Neurobiological studies of reading and reading disability. Journal of Communication Disorders, 34, 479-492. http://dx.doi.org/10.1016/S0021-9924(01)00060-0

Rastle, K., McCormick, S. F., Bayliss, L., \& Davis, C. J. (2011). Orthography influences the perception and production of speech. Journal of Experimental Psychology: Learning, Memory, and Cognition, 37, 15881594. http://dx.doi.org/10.1037/a0024833

Ravid, D. (2006). Hebrew orthography and literacy. In R. M. Joshi \& P. G. Aaron (Eds.), Handbook of orthography and literacy (pp. 339-364). Mahwah, NJ: Erlbaum.

R Core Team. (2018). R: A language and environment for statistical computing. Vienna, Austria: R Foundation for Statistical Computing. Retrieved from https://www.R-project.org/

Read, C., Zhang, Y. F., Nie, H. Y., \& Ding, B. Q. (1986). The ability to manipulate speech sounds depends on knowing alphabetic writing. Cognition, 24, 31-44. http://dx.doi.org/10.1016/0010-0277(86)90003-X

Reis, A., \& Castro-Caldas, A. (1997). Illiteracy: A cause for biased cognitive development. Journal of the International Neuropsychological Society, 3, 444-450. http://dx.doi.org/10.1017/S135561779700444X

Richardson, F. M., Seghier, M. L., Leff, A. P., Thomas, M. S., \& Price, C. J. (2011). Multiple routes from occipital to temporal cortices during reading. The Journal of Neuroscience, 31, 8239-8247. http://dx.doi.org/ 10.1523/JNEUROSCI.6519-10.2011

Rogers, H. (1995). Optimal orthographies. In I. Taylor \& D. R. Olson (Eds.), Scripts and literacy: Reading and learning to read alphabets, syllabaries, and characters (pp. 31-43). Dordrecht, NL: Kluwer. http:// dx.doi.org/10.1007/978-94-011-1162-1_3

Rogers, T. T., Lambon Ralph, M. A., Garrard, P., Bozeat, S., McClelland, J. L., Hodges, J. R., \& Patterson, K. (2004). Structure and deterioration of semantic memory: A neuropsychological and computational investigation. Psychological Review, 111, 205-235. http://dx.doi.org/10.1037/ 0033-295X.111.1.205

Rueckl, J. G., Paz-Alonso, P. M., Molfese, P. J., Kuo, W. J., Bick, A., Frost, S. J., . . Frost, R. (2015). Universal brain signature of proficient reading: Evidence from four contrasting languages. Proceedings of the National Academy of Sciences of the United States of America, 112, 15510-15515. http://dx.doi.org/10.1073/pnas.1509321112

Rumelhart, D. E., Hinton, G. E., \& Williams, R. J. (1986). Learning representations by back-propagating errors. Nature, 323, 533-536. http://dx.doi.org/10.1038/323533a0

Sampson, G. (1985). Writing systems: A linguistic introduction. Stanford, CA: Stanford University Press.

Scliar-Cabral, L., Morais, J., Nepomuceno, L., \& Kolinsky, R. (1997). The awareness of phonemes: So close-so far away. International Journal of Psycholinguistics, 13, 211-240.
Seeley, C. (1991). A history of writing in Japanese. Leiden, the Netherlands: Brill.

Seidenberg, M. S. (2011). Reading in different writing systems: One architecture, multiple solutions. In P. McCardle, J. Ren, \& O. Tzeng (Eds.), Dyslexia across languages: Orthography and the gene-brain behavior link (pp. 151-174). Baltimore, MD: Paul Brooke.

Seidenberg, M. S. (2013). The science of reading and its educational implications. Language Learning and Development, 9, 331-360. http:// dx.doi.org/10.1080/15475441.2013.812017

Seidenberg, M. S. (2017). Language at the speed of sight: How we read, why so many can't, and what can be done about it. New York, NY: Basic Books.

Seidenberg, M. S., \& McClelland, J. L. (1989). A distributed, developmental model of word recognition and naming. Psychological Review, 96, 523-568. http://dx.doi.org/10.1037/0033-295X.96.4.523

Serniclaes, W., Ventura, P., Morais, J., \& Kolinsky, R. (2005). Categorica perception of speech sounds in illiterate adults. Cognition, 98, B35-B44. http://dx.doi.org/10.1016/j.cognition.2005.03.002

Seymour, P. H., Aro, M., \& Erskine, J. M. (2003). Foundation literacy acquisition in European orthographies. British Journal of Psychology, 94, 143-174. http://dx.doi.org/10.1348/000712603321661859

Shany, M., Bar-On, A., \& Katzir, T. (2012). Reading different orthographic structures in the shallow-pointed Hebrew script: A cross-grade study in elementary school. Reading and Writing, 25, 1-22.

Share, D. L. (2008). On the Anglocentricities of current reading research and practice: The perils of overreliance on an "outlier" orthography. Psychological Bulletin, 134, 584-615. http://dx.doi.org/10.1037/00332909.134.4.584

Share, D. L. (2012). Frost and fogs, or sunny skies? Orthography, reading, and misplaced optimalism. Behavioral and Brain Sciences, 35, 307-308. http://dx.doi.org/10.1017/S0140525X12000271

Shaywitz, B. A., Shaywitz, S. E., Pugh, K. R., Mencl, W. E., Fulbright, R. K., Skudlarski, P., . . Gore, J. C. (2002). Disruption of posterior brain systems for reading in children with developmental dyslexia. Biological Psychiatry, 52, 101-110. http://dx.doi.org/10.1016/S0006-3223 (02)01365-3

Shu, H., Peng, H., \& McBride-Chang, C. (2008). Phonological awareness in young Chinese children. Developmental Science, 11, 171-181. http:// dx.doi.org/10.1111/j.1467-7687.2007.00654.x

Sibley, D. E., Kello, C. T., Plaut, D. C., \& Elman, J. L. (2008). Large-scale modeling of wordform learning and representation. Cognitive Science, 32, 741-754. http://dx.doi.org/10.1080/03640210802066964

Simons, G. F., \& Fennig, C. D. (Eds.). (2018). Ethnologue: Languages of the world (21st ed.). Dallas, TX: SIL International. Retrieved from http://www.ethnologue.com

Skeide, M. A., Kumar, U., Mishra, R. K., Tripathi, V. N., Guleria, A., Singh, J. P., . . Huettig, F. (2017). Learning to read alters corticosubcortical cross-talk in the visual system of illiterates. Science Advances, 3, e1602612. http://dx.doi.org/10.1126/sciadv.1602612

Smith, A. C., Monaghan, P., \& Huettig, F. (2014a). Literacy effects on language and vision: Emergent effects from an amodal shared resource (ASR) computational model. Cognitive Psychology, 75, 28-54. http:// dx.doi.org/10.1016/j.cogpsych.2014.07.002

Smith, A., Monaghan, P., \& Huettig, F. (2014b). Examining strains and symptoms of the 'Literacy Virus': The effects of orthographic transparency on phonological processing in a connectionist model of reading. In P. Bello, M. Guarini, M. McShane,\& B. Scassellati (Eds.), Proceedings of the 36th Annual Meeting of the Cognitive Science Society. Austin, TX: Cognitive Science Society.

Smith, J. S. (1996). Japanese writing. In P. T. Daniels \& W. Bright (Eds.) The world's writing systems. New York, NY: Oxford University Press. Snowling, M. J., \& Hulme, C. (Eds.). (2005). The science of reading: A handbook. Oxford, UK: Blackwell. 
Tan, L. H., Laird, A. R., Li, K., \& Fox, P. T. (2005). Neuroanatomical correlates of phonological processing of Chinese characters and alphabetic words: A meta-analysis. Human Brain Mapping, 25, 83-91. http:// dx.doi.org/10.1002/hbm.20134

Taylor, I., \& Olson, D. R. (Eds.). (1995). Scripts and literacy: Reading and learning to read alphabets, syllabaries, and characters. Dordrecht, NL: Kluwer. http://dx.doi.org/10.1007/978-94-011-1162-1

Taylor, J. S. H., Duff, F. J., Woollams, A., Monaghan, P., \& Ricketts, J. (2015). How word meaning influences word reading. Current Directions in Psychological Science, 24, 322-328. http://dx.doi.org/10.1177/ 0963721415574980

Taylor, J. S. H., Rastle, K., \& Davis, M. H. (2013). Can cognitive models explain brain activation during word and pseudoword reading? A metaanalysis of 36 neuroimaging studies. Psychological Bulletin, 139, 766791. http://dx.doi.org/10.1037/a0030266

The World Bank, World Development Indicators. (2016, August 23). Population total. Retrieved from http://data.worldbank.org/indicator/

The World Factbook, Central Intelligence Agency. (2016, August 23). Literacy: Total population. Retrieved from https://www.cia.gov/library/ publications/the-world-factbook/

Thuy, D. H. D., Matsuo, K., Nakamura, K., Toma, K., Oga, T., Nakai, T., ... Fukuyama, H. (2004). Implicit and explicit processing of kanji and kana words and non-words studied with fMRI. Neurolmage, 23, 878889. http://dx.doi.org/10.1016/j.neuroimage.2004.07.059

Tong, X., \& McBride-Chang, C. (2010). Chinese-English biscriptal reading: Cognitive component skills across orthographies. Reading and Writing, 23, 293-310. http://dx.doi.org/10.1007/s11145-009-9211-9

Tong, X., McBride-Chang, C., Shu, H., \& Wong, A. M. (2009). Morphological awareness, orthographic knowledge, and spelling errors: Keys to understanding early Chinese literacy acquisition. Scientific Studies of Reading, 13, 426-452. http://dx.doi.org/10.1080/10888430903162910

Treiman, R., \& Kessler, B. (2005). Writing systems and spelling development. In M. J. Snowling \& C. Hulme (Eds.), The science of reading: A handbook (pp. 120-134). Oxford, UK: Blackwell Publishing. http://dx .doi.org/10.1002/9780470757642.ch7

Treiman, R., \& Zukowski, A. (1991). Levels of phonological awareness. In S. A. Brady \& D. P. Shankweiler (Eds.), Phonological processes in literacy: A tribute to Isabelle Y. Liberman (pp. 67-83). Oxford, UK: Routledge.

Tsai, C. H. (1996). The Chinese character database (CCDB), Taiwan. Retrieved from http://technology.chtsai.org/charfreq/

Ueno, T., \& Lambon Ralph, M. A. (2013). The roles of the "ventral" semantic and "dorsal" pathways in conduite d'approche: A neuroanatomically-constrained computational modeling investigation. Frontiers in Human Neuroscience, 7, 422. http://dx.doi.org/10.3389/ fnhum.2013.00422

Ulicheva, A., Harvey, H., Aronoff, M., \& Rastle, K. (2020). Skilled readers' sensitivity to meaningful regularities in English writing. Cognition, 195, 103810. http://dx.doi.org/10.1016/j.cognition.2018.09.013

UNESCO Institute for Lifelong learning. (2016, August 23). Literacy and Language Classes in Community Centres, Country Profile: Japan. Retrieved from http://www.unesco.org/uil/litbase/?menu=14\&programme $=$ 131
UNESCO Institute for Statistics. (2016, August 23). Youth literacy rate, population 15-24 years. Retrieved from http://data.uis.unesco.org/

Vaessen, A., Bertrand, D., Tóth, D., Csépe, V., Faísca, L., Reis, A., \& Blomert, L. (2010). Cognitive development of fluent word reading does not qualitatively differ between transparent and opaque orthographies. Journal of Educational Psychology, 102, 827-842. http://dx.doi.org/10 .1037/a0019465

Venables, W. N., \& Ripley, B. D. (2002). Modern Applied Statistics with $S$ (4th ed.). New York, NY: Springer. http://dx.doi.org/10.1007/978-0387-21706-2

Venetzky, R. L. (1970). Principles for the design for practical writing systems. Anthropological Linguistics, 12, 256-270.

Weiss, Y., Katzir, T., \& Bitan, T. (2015). Many ways to read your vowels-Neural processing of diacritics and vowel letters in Hebrew. NeuroImage, 121, 10-19. http://dx.doi.org/10.1016/j.neuroimage.2015 .07 .029

Winskel, H., \& Iemwanthong, K. (2010). Reading and spelling acquisition in Thai children. Reading and Writing, 23, 1021-1053. http://dx.doi.org/ 10.1007/s11145-009-9194-6

Yang, J., McCandliss, B. D., Shu, H., \& Zevin, J. D. (2009). Simulating language-specific and language-general effects in a statistical learning model of Chinese reading. Journal of Memory and Language, 61, 238-257. http://dx.doi.org/10.1016/j.jml.2009.05.001

Yang, J., Shu, H., McCandliss, B. D., \& Zevin, J. D. (2013). Orthographic influences on division of labor in learning to read Chinese and English: Insights from computational modeling. Bilingualism: Language and Cognition, 16, 354-366. http://dx.doi.org/10.1017/S1366728912000296

Yang, J., Zevin, J. D., Shu, H., McCandliss, B. D., \& Li, P. (2006). A "triangle model" of Chinese reading. Proceedings of the 28th Annual Conference of the Cognitive Science Society. Mahwah, NJ: Erlbaum.

Zhou, Y. G. (1978). To what extent are the "phonetics" of present-day Chinese characters still phonetic? Zhongguo Yuwen, 146, 172-177.

Zhou, Y., McBride, C., Leung, J. S. M., Wang, Y., Joshi, M., \& Farver, J. (2018). Chinese and English reading-related skills in L1 and L2 Chinesespeaking children in Hong Kong. Language, Cognition and Neuroscience, 33, 300-312. http://dx.doi.org/10.1080/23273798.2017.1342848

Ziegler, J. C., Bertrand, D., Tóth, D., Csépe, V., Reis, A., Faísca, L., . . Blomert, L. (2010). Orthographic depth and its impact on universal predictors of reading: A cross-language investigation. Psychological Science, 21, 551-559. http://dx.doi.org/10.1177/0956797610363406

Ziegler, J. C., \& Ferrand, L. (1998). Orthography shapes the perception of speech: The consistency effect in auditory word recognition. Psychonomic Bulletin \& Review, 5, 683-689. http://dx.doi.org/10.3758/ BF03208845

Ziegler, J. C., \& Goswami, U. (2005). Reading acquisition, developmental dyslexia, and skilled reading across languages: A psycholinguistic grain size theory. Psychological Bulletin, 131, 3-29. http://dx.doi.org/10 1037/0033-2909.131.1.3

Received September 5, 2016

Revision received June 28, 2020 Accepted June 30, 2020 